\begin{tabular}{|c|l|}
\hline Title & On the inviscid limit problem of the vorticity equations for viscous incompressible flows in the half plane \\
\hline Author(s) & Maekawa, Y asunori \\
\hline Citation & Hokkaido University Preprint Series in Mathematics, 1005, 1-50 \\
\hline Issue Date & 2012-4-19 \\
\hline DOI & 10.14943/84151 \\
\hline Doc URL & http://hdl.handle.net/2115/69810 \\
\hline Type & bulletin (article) \\
\hline File Information & pre1005.pdf \\
\hline
\end{tabular}

Instructions for use 


\title{
On the inviscid limit problem of the vorticity equations for viscous incompressible flows in the half plane
}

\author{
Yasunori Maekawa \\ Department of Mathematics, Kobe University \\ 1-1 Rokkodai, Nada-ku, Kobe 657-8501, Japan \\ yasunori@math.kobe-u.ac.jp
}

\begin{abstract}
We consider the Navier-Stokes equations for viscous incompressible flows in the half plane under the no-slip boundary condition. By using the vorticity formulation we prove the (local in time) convergence of the Navier-Stokes flows to the Euler flows outside a boundary layer and to the Prandtl flows in the boundary layer at the inviscid limit when the initial vorticity is located away from the boundary.
\end{abstract}

Keywords Navier-Stokes equations; Vorticity equations; No-slip boundary conditions; Inviscid limit 2010 Mathematics Subject Classification 35Q30; 76D05; 76D10

\section{Introduction}

In this paper we consider the Navier-Stokes equations for viscous incompressible flows in the half plane under the no-slip boundary conditions:

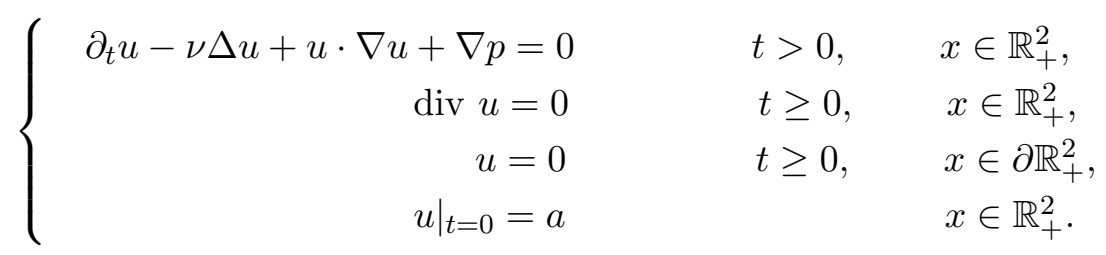

Here $\mathbb{R}_{+}^{2}=\left\{\left(x_{1}, x_{2}\right) \in \mathbb{R}^{2} \mid x_{2}>0\right\}$ and $\nu$ is the kinematic viscosity which is assumed to be a positive constant, and $u=u(t, x)=\left(u_{1}(t, x), u_{2}(t, x)\right), p=p(t, x)$ denote the velocity field, the pressure field, respectively. We will use the standard notations for derivatives; $\partial_{t}=\partial / \partial t, \partial_{j}=\partial / \partial x_{j}, \Delta=\sum_{j=1}^{2} \partial_{j}^{2}$, $\operatorname{div} u=\sum_{j=1}^{2} \partial_{j} u_{j}$, and $u \cdot \nabla u=\sum_{j=1}^{2} u_{j} \partial_{j} u$.

The behavior of viscous incompressible flows at the inviscid limit is a classical issue in the fluid dynamics. When the fluid domain has no boundary it is well known that the solution of the NavierStokes equations converges to the one of the Euler equations, e.g. [8, 6, 9, 22]. However, in the presence of nontrivial boundary one is faced with a serious difficulty in this problem even in the two-dimensional case if the no-slip boundary condition is imposed on the velocity field. This is due to the appearance of the boundary layer, whose formation is formally explained by Prandtl's theory that estimates the thickness of the boundary layer as the square root of the viscosity. So far the rigorous verification of Prandtl's boundary layer theory was achieved only for some specific cases. For example, it is proved in $[2,32,33]$ that for analytic initial data the solution of $\left(\mathrm{NS}_{\nu}\right)$ converges to the one of the Euler equations outside the boundary layer and to the one of the Prandtl equations in the boundary layer. When the domain 
and the initial data possess a circular symmetry the significant cancellation occurs in the nonlinear term, and hence the convergence is affirmatively justified; see $[23,4,18,19,15,25]$. On the other hand, the necessary and sufficient condition for the $L^{2}$ convergence of the Navier-Stokes flows to the Euler flows was given by [13], which was extended by several authors [35, 37, 14, 15].

In the fluid dynamics the vorticity field, i.e., the curl of the velocity field, is also an important quantity and useful in understanding various phenomena. At the inviscid limit it is recognized that the vorticity is highly produced in the boundary layer and forms a vortex sheet (or line in the two dimension) along the boundary. However, under the no-slip boundary condition on the velocity field the study of the vorticity field is still less developed mathematically, since the vorticity is subject to a nonlocal and nonlinear boundary condition from which it is not easy to derive useful informations. This is contrasting with the case of the whole plane, where the detailed analysis has been established [21, 7]. In the case of the half plane the situation is relaxed a little, since the solution formula is available for the linearized problem. By making use of this solution formula, [20] studied the vorticity equations in the half plane and established some asymptotic estimates which hold at least up to the time $\mathcal{O}\left(\nu^{1 / 3}\right)$ for $0<\nu \ll 1$.

The aim of this paper is to study the inviscid limit of $\left(\mathrm{NS}_{\nu}\right)$ by using the vorticity formulation in [20] when the initial vorticity is located away from the boundary. This class of initial data includes a dipole-type localized vortex, which is often used in numerical works as a benchmark to investigate the interaction between the vorticity created on the boundary and the original vorticity away from the boundary; cf. [30, 16, 28]. In this paper we will establish the asymptotic expansion of vorticity fields at the inviscid limit for a short time $T>0$ (but $T$ is independent of the viscosity), that is of the form

$$
\omega^{(\nu)}(t, x)=\omega_{E}(t, x)+\frac{1}{\nu^{\frac{1}{2}}} w_{P}\left(t, x_{1}, \frac{x_{2}}{\nu^{\frac{1}{2}}}\right)+\frac{1}{\nu^{\frac{1}{2}}} w_{I P}^{(\nu)}\left(t, x_{1}, \frac{x_{2}}{\nu^{\frac{1}{2}}}\right)+w_{I I}^{(\nu)}(t, x) .
$$

Here $\omega^{(\nu)}$ is the vorticity field of the Navier-Stokes flows $\left(\mathrm{NS}_{\nu}\right), \omega_{E}$ is the vorticity field of the Euler flows (see (E) below), $w_{P}$ is the vorticity field of the Prandtl flows (see $(\mathrm{P})$ below), and the remainder parts $w_{I P}^{(\nu)}, w_{I I}^{(\nu)}$ are of the order $\mathcal{O}\left(\nu^{1 / 2}\right)$ in suitable norms. It should be noted here that, even if there is no vorticity near the boundary at the initial time, the vorticity is immediately created there and forms a vortex line along the boundary in positive time. In particular, we have to deal with the boundary layer and the infinite growth of vorticity at the inviscid limit. Although we will focus on the analysis of the vorticity field in this paper, the asymptotic expansion for the velocity field is easily obtained from the Biot-Savart law. More precisely, we have the following

Theorem 1.1 Assume that the initial velocity $a=\left(a_{1}, a_{2}\right)$ belongs to $\dot{W}_{0, \sigma}^{1, p}\left(\mathbb{R}_{+}^{2}\right)$ for some $1<p<2$ and the initial vorticity $b=\partial_{1} a_{2}-\partial_{2} a_{1}$ belongs to $W^{4,1}\left(\mathbb{R}_{+}^{2}\right) \cap W^{4,2}\left(\mathbb{R}_{+}^{2}\right)$. Assume also that

$$
d_{0}=\operatorname{dist}\left(\partial \mathbb{R}_{+}^{2}, \operatorname{supp} b\right)>0 .
$$

Then there are positive constants $C$ and $T$ such that the following estimate holds for $0<\nu \ll 1$.

$$
\sup _{0<t<T}\left\|u_{N S}^{(\nu)}(t)-u_{E}(t)-u_{P}^{(\nu)}(t)\right\|_{L^{\infty}\left(\mathbb{R}_{+}^{2}\right)} \leq C \nu^{\frac{1}{2}} .
$$

Here $u_{N S}^{(\nu)}$ is the solution of $\left(\mathrm{NS}_{\nu}\right), u_{E}$ is the solution of the Euler equations with the initial velocity a, and $u_{P}^{(\nu)}$ describes the boundary layer of the form

$$
u_{P}^{(\nu)}(t, x)=\left(v_{P, 1}\left(t, x_{1}, \frac{x_{2}}{\nu^{\frac{1}{2}}}\right), \nu^{\frac{1}{2}} v_{P, 2}\left(t, x_{1}, \frac{x_{2}}{\nu^{\frac{1}{2}}}\right)\right),
$$

where $v_{P}=\left(v_{P, 1}, v_{P, 2}\right)$ is the solution of the (modified) Prandtl equations. Moreover, $T$ is estimated from below as $T \geq c \min \left\{d_{0}, 1\right\}$, where $c$ is a positive constant depending only on $\|b\|_{W^{4,1}\left(\mathbb{R}_{+}^{2}\right) \cap W^{4,2}\left(\mathbb{R}_{+}^{2}\right)}$. 
The space $\dot{W}_{0, \sigma}^{1, p}\left(\mathbb{R}_{+}^{2}\right)$ is the completion with respect to the norm $\|\nabla \cdot\|_{L^{p}\left(\mathbb{R}_{+}^{2}\right)}$ of the space of all smooth, divergence-free vector fields with compact support in $\mathbb{R}_{+}^{2}$, and $W^{k, p}\left(\mathbb{R}_{+}^{2}\right)$ is a usual Sobolev space.

The velocity field $u_{E}=\left(u_{E, 1}, u_{E, 2}\right)$ of the ideal incompressible flows is subject to the Euler equations

$$
\left\{\begin{array}{rlrl}
\partial_{t} u_{E}+u_{E} \cdot \nabla u_{E}+\nabla p_{E} & =0 & t>0, & x \in \mathbb{R}_{+}^{2}, \\
\operatorname{div} u_{E}=0 & t \geq 0, & x \in \mathbb{R}_{+}^{2}, \\
u_{E, 2}=0 & t \geq 0, & x \in \partial \mathbb{R}_{+}^{2}, \\
\left.u_{E}\right|_{t=0}=a & & x \in \mathbb{R}_{+}^{2} .
\end{array}\right.
$$

Since the initial velocity $a$ in Theorem 1.1 possesses an enough regularity the existence and the uniqueness of the classical solution of (E) are verified by the known approach [38, 39, 12, 3].

The Prandtl equations for the boundary layer profile $\tilde{v}_{P}=\left(\tilde{v}_{P, 1}, \tilde{v}_{P, 2}\right)$ are written as follows.

$$
\left\{\begin{array}{rlrl}
\left(\partial_{t}-\partial_{X_{2}}^{2}\right) \tilde{v}_{P, 1}+\tilde{v}_{P, 1} \partial_{1} \tilde{v}_{P, 1}+\tilde{v}_{P, 2} \partial_{X_{2}} \tilde{v}_{P, 1}+\partial_{1} \tilde{\pi}_{P}=0 & t>0, & \left(x_{1}, X_{2}\right) \in \mathbb{R}_{+}^{2} \\
\partial_{1} \tilde{v}_{P, 1}+\partial_{X_{2}} \tilde{v}_{P, 2}=0, \quad \partial_{X_{2}} \tilde{\pi}_{P}=0 & t \geq 0, & \left(x_{1}, X_{2}\right) \in \mathbb{R}_{+}^{2} \\
\tilde{v}_{P}\left(t, x_{1}, 0\right)=0 & t \geq 0, & x_{1} \in \mathbb{R}, \\
\lim _{X_{2} \rightarrow \infty} \tilde{v}_{P, 1}\left(t, x_{1}, X_{2}\right)=u_{E, 1}\left(t, x_{1}, 0\right) & & t \geq 0, & x_{1} \in \mathbb{R}, \\
\lim _{X_{2} \rightarrow \infty} \tilde{\pi}_{P}\left(t, x_{1}, X_{2}\right)=p_{E}\left(t, x_{1}, 0\right) & t \geq 0, & x_{1} \in \mathbb{R}, \\
\left.\tilde{v}_{P}\right|_{t=0}=0 & & & \left(x_{1}, X_{2}\right) \in \mathbb{R}_{+}^{2} .
\end{array}\right.
$$

The local solvability of the Prandtl equations is proved by $[29,24]$ under some assumptions on the monotonicity of the data, and by $[2,32]$ for the analytic initial data. The analyticity condition is in fact required only in the tangential direction [17]. But the solvability for general initial data in a Sobolev class is still an open problem. The velocity field $v_{P}=\left(v_{P, 1}, v_{P, 2}\right)$ for the modified Prandtl equations is defined by $v_{P, 1}\left(t, x_{1}, X_{2}\right)=\tilde{v}_{P, 1}\left(t, x_{1}, X_{2}\right)-u_{E, 1}\left(t, x_{1}, 0\right), v_{P, 2}\left(t, x_{1}, X_{2}\right)=\int_{X_{2}}^{\infty} \partial_{1} v_{P, 1}\left(t, x_{1}, Y_{2}\right) \mathrm{d} Y_{2}$; cf. [33].

Theorem 1.1 is derived from the analysis of the vorticity equations which will be stated in the next section. The lower bound of the time $T$ in Theorem 1.1 is of the order $\mathcal{O}\left(d_{0}\right)$ when $d_{0}$ is small, which seems to be natural and optimal to ensure (1.3) in our setting, since our initial data is not necessarily analytic in the region away from the boundary. After the time period ensured by Theorem 1.1 the separation of the boundary layer is expected to occur in general and the vorticity will exhibit rather complicated behaviors; $[16,28]$. The mathematical understanding of these phenomena is a challenging problem.

In the rest of this section let us briefly describe the idea to establish the asymptotic expansion (1.3). The proof is based on two key observations. Firstly we observe that the solution should be analytic at least near the boundary because so is at the initial time. Thus the solvability of the Prandtl equations itself is not surprising in our setting; cf. $[2,32,17]$. But we note here that the solvability of the Prandtl equations does not necessarily imply the desired asymptotic expansion, as in the counter example by [10]. Moreover, our solution should lose the analyticity as it leaves the boundary, and it is important to estimate how to lose it precisely. We overcome this difficulty by introducing a suitable weighted function space which represents this loss of analyticity. Secondly we use the fact that the vorticity field of the Euler flows satisfies the transport equations and hence its support is away from the boundary even in positive time. Then the vorticity of the Navier-Stokes flows is expected to be small exponentially in $\nu^{-1}$ in the region between the boundary layer and the support of the vorticity of the Euler flows. The presence of this region prevents the strong and uncontrollable interaction of the vorticity produced in the boundary layer with the vorticity originated from the initial one, resulting the classical thickness $\mathcal{O}\left(\nu^{1 / 2}\right)$ of the boundary layer at least for a short time. A suitable weighted function space has to be introduced again in 
order to describe this region. In this step we also appeal to the result [5] on the sharp pointwise estimate for fundamental solutions of the linear heat-transport equations in the whole space. After establishing the estimates for some linear and bilinear mappings we construct the solution by applying the abstract Cauchy Kowalewski (ACK) theorem as in the previous works $[2,32,33]$. The ACK theorem used in this paper is a slightly extended version of $[27,11]$. Due to the lack of the analyticity away from the boundary the construction of the remainder part in the asymptotic expansion requires intricate calculations. In particular, the iteration sequence, for which the ACK theorem is applied, has to be defined in a technical manner; see Section 4.

The rest of this paper is organized as follows. In Section 2.1 we recall the vorticity equations for $\left(\mathrm{NS}_{\nu}\right),(\mathrm{E})$, and $(\mathrm{P})$, together with the appropriate boundary conditions. In Section 2.2 we state the integral formula for the linearized problem related with the vorticity equations for $\left(\mathrm{NS}_{\nu}\right)$. In Section 2.3 we introduce the wighted function spaces which play central roles in this work. The estimates for the Biot-Savart law in these function spaces are obtained in Section 2.4. Section 3 takes a large part of this paper, where we collect the estimates for a number of linear and bilinear mappings. Based on these estimates we establish the asymptotic expansion of vorticity fields in Section 4 by solving suitable integral equations with the aid of the ACK theorem. In particular, the boundary layer part is constructed in Theorems 4.4, 4.12, and the remainder part is obtained in Theorem 4.10. Theorem 1.1 is finally proved in Section 5. We state some open problems related to this work in Section 6. Some of the key estimates and the result on the fundamental solution of the heat-transport equations are stated in the appendix.

Finally we give some comments on the notations used in this paper. We write $\alpha \lesssim \beta$ when $\alpha \leq C \beta$ holds with a numerical constant $C>0$ (independent of $\nu, d_{0}$, and so on). We also write $\alpha \lesssim\left\{\begin{array}{l}\beta_{1} \\ \beta_{2}\end{array}\right\} \gamma$ when both $\alpha \lesssim \beta_{1} \gamma$ and $\alpha \lesssim \beta_{2} \gamma$ hold. For $d_{E}>0$ (defined by (2.3) below) and $l>0$ we define smooth nonnegative cut-off functions $\chi_{l d_{E}}\left(x_{2}\right)$ and $\chi_{l d_{E}}^{c}\left(x_{2}\right)$ by

$$
\chi_{l d_{E}}\left(x_{2}\right)=\left\{\begin{array}{ll}
1 & \text { if } 0 \leq x_{2} \leq l d_{E}, \\
0 & \text { if } x_{2} \geq(l+1) d_{E},
\end{array} \quad \chi_{l d_{E}}^{c}\left(x_{2}\right)=1-\chi_{l d_{E}}\left(x_{2}\right), \quad\left|\chi_{l d_{E}}^{(k)}\left(x_{2}\right)\right| \leq C d_{E}^{-k} .\right.
$$

When $A$ is a measurable set in $\mathbb{R}_{+}^{2}$ we also denote by $\chi_{A}$ the characteristic function of $A$.

\section{Preliminaries}

\subsection{Vorticity equations}

Let $\omega=$ Rot $u=\partial_{1} u_{2}-\partial_{2} u_{1}$ be the vorticity field. Then the Biot-Sawart law in $\mathbb{R}_{+}^{2}$ is given by

$$
u=J(\omega)=\left(J_{1}(\omega), J_{2}(\omega)\right):=\nabla^{\perp}\left(-\Delta_{D}\right)^{-1} \omega,
$$

where $\nabla^{\perp}=\left(\partial_{2},-\partial_{1}\right)$ and $h=\left(-\Delta_{D}\right)^{-1} f$ denotes the solution of the Poisson equation $-\Delta h=f$ in $\mathbb{R}_{+}^{2}$ and $h=0$ on $\partial \mathbb{R}_{+}^{2}$. We introduce the bilinear forms

$$
B(f, h)=J(f) \cdot \nabla h, \quad N(f, h)=\left.J_{1}(B(f, h))\right|_{x_{2}=0} .
$$

Then the vorticity equations for the Navier-Stokes flows are described as follows.

$$
\left\{\begin{aligned}
\partial_{t} \omega-\nu \Delta \omega+B(\omega, \omega) & =0 \\
\nu\left(\partial_{2} \omega+\left(-\partial_{1}^{2}\right)^{\frac{1}{2}} \omega\right) & =-N(\omega, \omega) \\
\left.\omega\right|_{t=0} & =b:=\operatorname{Rot} a
\end{aligned}\right.
$$

$$
\begin{array}{ll}
t>0, & x \in \mathbb{R}_{+}^{2}, \\
t>0, & x \in \partial \mathbb{R}_{+}^{2}, \\
& x \in \mathbb{R}_{+}^{2} .
\end{array}
$$


The first equation of $\left(\mathrm{V}_{\nu}\right)$ is obtained by taking the Rot in the first equation of $\left(\mathrm{NS}_{\nu}\right)$. The boundary condition in $\left(\mathrm{V}_{\nu}\right)$ is imposed so as to keep the no-slip boundary condition on $u=J(\omega) ;[1,20]$.

The vorticity field of the Euler flows, denoted by $\omega_{E}$, satisfies the equations

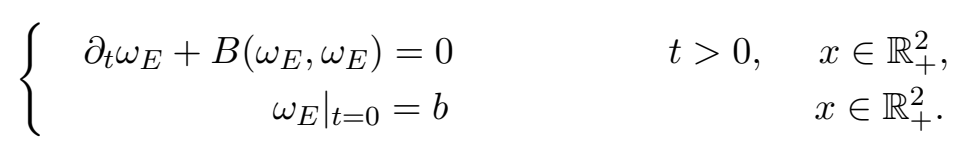

When $b \in W^{4,1}\left(\mathbb{R}_{+}^{2}\right) \cap W^{4,2}\left(\mathbb{R}_{+}^{2}\right)$ it is not difficult to show that the classical solution of $\left(\mathrm{V}_{\mathrm{E}}\right)$ exists globally in time and $\omega_{E} \in C^{1}\left([0, T] \times \overline{\mathbb{R}_{+}^{2}}\right) \cap L^{\infty}\left(0, T ; W^{4,1}\left(\mathbb{R}_{+}^{2}\right) \cap W^{4,2}\left(\mathbb{R}_{+}^{2}\right)\right)$. Moreover, since $d_{0}=$ $\operatorname{dist}\left(\partial \mathbb{R}_{+}^{2}, \operatorname{supp} b\right)>0$ we have

$$
\cup_{0 \leq t \leq T_{0}} \operatorname{supp} \omega(t) \subset\left\{x \in \mathbb{R}_{+}^{2} \mid x_{2} \geq 2^{5} d_{E}\right\}, \quad d_{E}=\min \left\{2^{-6} d_{0}, 2^{-1}\right\}
$$

for some $T_{0} \geq C d_{E}$ with $C>0$ depending only on $\|b\|_{W^{4,1} \cap W^{4,2}}$.

The vorticity field of the Prandtl flows $\tilde{v}_{P}$ is given by $w_{P}=-\partial_{2} \tilde{v}_{P, 1}$ and the Biot-Sawart law in this case is written as

$$
\begin{aligned}
\tilde{v}_{P, 1}\left(t, x_{1}, X_{2}\right) & =v_{E, 1}\left(t, x_{1}, X_{2}\right)+v_{P, 1}\left(t, x_{1}, X_{2}\right):=u_{E, 1}\left(t, x_{1}, 0\right)+\int_{X_{2}}^{\infty} w_{P}\left(t, x_{1}, Y_{2}\right) d Y_{2} \\
\tilde{v}_{P, 2}\left(t, x_{1}, X_{2}\right) & =v_{E, 2}\left(t, x_{1}, X_{2}\right)+v_{P, 2}\left(t, x_{1}, X_{2}\right) \\
& :=X_{2} \partial_{2} u_{E, 2}\left(t, x_{1}, 0\right)-\partial_{1}\left(\int_{0}^{X_{2}} Y_{2} w_{P}\left(t, x_{1}, Y_{2}\right) d Y_{2}+X_{2} \int_{X_{2}}^{\infty} w_{P}\left(t, x_{1}, Y_{2}\right) d Y_{2}\right) .
\end{aligned}
$$

Set $\nabla_{X}=\left(\partial_{1}, \partial_{X_{2}}\right)$. Then the equation for $w_{P}=w_{P}\left(t, x_{1}, X_{2}\right)$ is given by

$$
\left\{\begin{array}{rlrl}
\partial_{t} w_{P}-\partial_{X_{2}}^{2} w_{P}=-\tilde{v}_{P} \cdot \nabla_{X} w_{P} & t>0, & \left(x_{1}, X_{2}\right) \in \mathbb{R}_{+}^{2}, \\
\partial_{X_{2}} w_{P}=-\int_{0}^{\infty} \tilde{v}_{P} \cdot \nabla_{X} w_{P} d Y_{2}-N\left(\omega_{E}, \omega_{E}\right) & t>0, & \left(x_{1}, X_{2}\right) \in \partial \mathbb{R}_{+}^{2}, \\
\left.w_{P}\right|_{t=0}=0 & \left(x_{1}, X_{2}\right) \in \mathbb{R}_{+}^{2} .
\end{array}\right.
$$

The boundary condition of $w_{P}$ is derived from the same argument as in $\left(\mathrm{V}_{\nu}\right)$ (cf. [1]), or one can deduce it also by performing the formal expansion $\omega(t, x)=\omega_{E}(t, x)+\nu^{-1 / 2} w_{P}\left(t, x_{1}, x_{2} / \nu^{1 / 2}\right)+$ remainder. To establish the rigorous asymptotic expansion of $\omega=\omega^{(\nu)}$ we first aim the decomposition $\omega^{(\nu)}=$ $\omega_{E}+\omega_{B}^{(\nu)}+\omega_{I}^{(\nu)}$, where $\omega_{B}^{(\nu)}, \omega_{I}^{(\nu)}$ are solutions of the equations

$$
\begin{aligned}
& \left\{\begin{array}{rlrl}
\partial_{t} \omega_{B}-\nu \Delta \omega_{B}+B\left(\omega_{E}+\omega_{B}, \omega_{B}\right) & =0 & t>0, & x \in \mathbb{R}_{+}^{2}, \\
\nu\left(\partial_{2} \omega_{B}+\left(-\partial_{1}^{2}\right)^{\frac{1}{2}} \omega_{B}\right) & =-N\left(\omega_{E}+\omega_{B}, \omega_{B}\right)-N\left(\omega_{E}, \omega_{E}\right) & t>0, & x \in \partial \mathbb{R}_{+}^{2}, \quad\left(\mathrm{~V}_{\mathrm{B}_{\nu}}\right) \\
\left.\omega_{B}\right|_{t=0} & =0 & & x \in \mathbb{R}_{+}^{2},
\end{array}\right. \\
& \left\{\begin{array}{rlrl}
\partial_{t} \omega_{I}-\nu \Delta \omega_{I} & =-B\left(\omega, \omega_{I}\right)-B\left(\omega_{I}, \omega_{E}+\omega_{B}\right)-B\left(\omega_{B}, \omega_{E}\right)+\nu \Delta \omega_{E} & t>0, & x \in \mathbb{R}_{+}^{2}, \\
\nu\left(\partial_{2} \omega_{I}+\left(-\partial_{1}^{2}\right)^{\frac{1}{2}} \omega_{I}\right) & =-N\left(\omega, \omega_{I}\right)-N\left(\omega_{I}, \omega_{E}+\omega_{B}\right)-N\left(\omega_{B}, \omega_{E}\right)+\nu J_{1}\left(\Delta \omega_{E}\right) & t>0, & x \in \partial \mathbb{R}_{+}^{2}, \\
\left.\omega_{I}\right|_{t=0} & =0 & & x \in \mathbb{R}_{+}^{2},
\end{array}\right.
\end{aligned}
$$

respectively. Here we have used $J_{1}(\Delta f)=-\partial_{2} f-\left(-\partial_{1}^{2}\right)^{1 / 2} f$ on $\partial \mathbb{R}_{+}^{2}$. In $\left(\mathrm{V}_{\mathrm{B}_{\nu}}\right)$ and $\left(\mathrm{V}_{\mathrm{I}_{\nu}}\right)$ the symbol $(\nu)$ is abbreviated in the notations of $\omega, \omega_{B}$, and $\omega_{I}$, for simplicity. The function $\omega_{B}$ takes the form $\omega_{B}=R_{1 / \nu} w_{B}$ for a suitable profile function $w_{B}=w_{B}^{(\nu)}$, where $R_{s}$ is the scaling operator defined by

$$
\left(R_{s} f\right)(x)=s^{\frac{1}{2}} f\left(x_{1}, s^{\frac{1}{2}} x_{2}\right), \quad s>0 .
$$


The function $w_{B}^{(\nu)}$ will be shown to converge to the solution $w_{P}$ of $\left(\mathrm{V}_{\mathrm{p}}\right)$ in the limit $\nu \rightarrow 0$ (Theorem 4.13). We will construct $\omega_{I}$ of the form $\omega_{I}=R_{1 / \nu} w_{I B}+w_{I I}$ for some functions $w_{I B}=w_{I B}^{(\nu)}$ and $w_{I I}=w_{I I}^{(\nu)}$. The proof for the existence of such $w_{B}, w_{I B}$, and $w_{I I}$ is given in Section 4 (Theorems 4.4, 4.10) by solving the associated integral equations with the aid of the ACK theorem.

\subsection{Representation formula for solutions of the linearized problem}

In this section we recall the solution formula to the linear problem

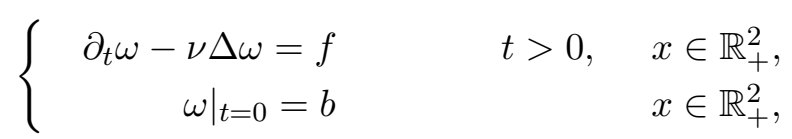

subject to the boundary condition

$$
\nu\left(\partial_{2}+\left(-\partial_{1}^{2}\right)^{\frac{1}{2}}\right) \omega=g \quad t>0, \quad x \in \partial \mathbb{R}_{+}^{2} .
$$

Here $f, g, b$ are assumed to be smooth and decay fast enough at spatial infinity. We denote by $G$ and $E$ the two-dimensional Gaussian and Newton potential, respectively, i.e., $G(t, x)=(4 \pi t)^{-1} \exp \left(-|x|^{2} /(4 t)\right)$ and $E(x)=-(2 \pi)^{-1} \log |x|$. Let $*$ be the standard convolution in $\mathbb{R}^{2}$. Following [20], we set

$$
\Gamma(t, x)=(\Xi E * G(t))(x), \quad \Xi=2\left(\partial_{1}^{2}+\left(-\partial_{1}^{2}\right)^{\frac{1}{2}} \partial_{2}\right) .
$$

We also use the notation $\left(h_{1} \star h_{2}\right)(x)=\int_{\mathbb{R}_{+}^{2}} h_{1}\left(x-y^{*}\right) h_{2}(y) \mathrm{d} y$, where $y^{*}=\left(y_{1},-y_{2}\right)$.

Lemma 2.1 ([20]) The integral equation for $(L V)-(L B C)$ is given by

$$
\begin{aligned}
\omega(t)= & e^{\nu t \Delta_{N}} b+\Gamma(\nu t) \star b-\Gamma(0) \star b \\
& +\int_{0}^{t} e^{\nu(t-s) \Delta_{N}}\left(f(s)-g(s) \mathcal{H}_{\left\{x_{2}=0\right\}}^{1}\right) \mathrm{d} s+\int_{0}^{t} \Gamma(\nu(t-s)) \star\left(f(s)-g(s) \mathcal{H}_{\left\{x_{2}=0\right\}}^{1}\right) \mathrm{d} s \\
& -\int_{0}^{t} \Gamma(0) \star\left(f(s)-g(s) \mathcal{H}_{\left\{x_{2}=0\right\}}^{1}\right) \mathrm{d} s .
\end{aligned}
$$

Here $e^{t \Delta_{N}}$ is the semigroup for the heat equation (with the unit viscosity) in $\mathbb{R}_{+}^{2}$ subject to the homogeneous Neumann boundary condition, $\Gamma(0) \star:=\lim _{t \downarrow 0} \Gamma(t) \star$, and $g \mathcal{H}_{\left\{x_{2}=0\right\}}^{1}$ is a one-dimensional Hausdorff measure with density $g$ defined by $\left\langle h, g \mathcal{H}_{\left\{x_{2}=0\right\}}^{1}\right\rangle=\int_{\mathbb{R}} h\left(x_{1}, 0\right) g\left(x_{1}\right) \mathrm{d} x_{1}$ for $h \in C_{0}\left(\overline{\mathbb{R}_{+}^{2}}\right)$.

The reader is referred to $[34,36]$ for the solution formula of the (Navier-)Stokes equations. We note that $\Gamma(0) \star h=\Xi E \star h$ in $\mathbb{R}_{+}^{2}$. The following cancellation property is important.

Lemma 2.2 If $g=\left.J_{1}(f)\right|_{x_{2}=0}$ then $\Xi E \star\left(f-g \mathcal{H}_{\left\{x_{2}=0\right\}}^{1}\right)=0$ in $\mathbb{R}_{+}^{2}$. In particular, we have $\Xi E \star b=0$ in $\mathbb{R}_{+}^{2}$ if $J_{1}(b)=0$ on $\partial \mathbb{R}_{+}^{2}$.

For the proof of Lemma 2.2, see [20, Proposition 3.2]. We will also use

Lemma 2.3 The following identity holds.

$$
\begin{aligned}
& \int_{0}^{t} \Gamma(\nu(t-s)) \star\left(f(s)-g(s) \mathcal{H}_{\left\{x_{2}=0\right\}}^{1}\right) \mathrm{d} s-\int_{0}^{t} \Gamma(0) \star\left(f(s)-g(s) \mathcal{H}_{\left\{x_{2}=0\right\}}^{1}\right) \mathrm{d} s \\
= & -\nu \int_{0}^{t} \int_{0}^{s} \Xi G(\nu(s-\tau)) \star\left(f(\tau)-g(\tau) \mathcal{H}_{\left\{x_{2}=0\right\}}^{1}\right) \mathrm{d} \tau \mathrm{d} s .
\end{aligned}
$$


Lemma 2.3 follows from the definition of $\Gamma(t, x)$ and the equality $G(t)=-E * \partial_{t} G(t)$. The right-hand side of (2.9) is useful in studying the spatial decay, while the left-hand side of (2.9) has an advantage in view of regularity when the second term vanishes. This property will be taken into account in the definition of the solution mapping in Section 4.

\section{$2.3 \quad$ Function spaces}

We will construct $\omega_{B}$ and $\omega_{I}$ by applying the ACK theorem. For this purpose it is essential to set up a suitable family of Banach spaces. Recalling the definition of $d_{E} \in(0,1 / 2)$ in $(2.3)$, we set

$$
\begin{aligned}
\varphi_{B}^{(\mu, \rho)}\left(\xi_{1}, X_{2}\right) & =\varphi_{B_{\nu}}^{(\mu, \rho)}\left(\xi_{1}, X_{2}\right)=\exp \left(\frac{\left(\mu-\nu^{\frac{1}{2}} X_{2}\right)_{+}\left|\xi_{1}\right|}{4}+\rho X_{2}^{2}\right) \\
\varphi_{I}^{(\mu, \theta)}\left(\xi_{1}, x_{2}\right) & =\varphi_{I_{\nu}}^{(\mu, \theta)}\left(\xi_{1}, x_{2}\right)=\exp \left(\frac{\left(\mu-x_{2}\right)_{+}\left|\xi_{1}\right|}{4}+\frac{\theta}{\nu}\left(6 d_{E}-x_{2}\right)_{+}^{2}\right)
\end{aligned}
$$

where $\mu, \rho, \theta \geq 0$ and $(\alpha)_{+}=\max \{\alpha, 0\}$ for $\alpha \in \mathbb{R}$. Let

$$
\left\langle\xi_{1}\right\rangle=\left(1+\xi_{1}^{2}\right)^{\frac{1}{2}}, \quad \hat{f}\left(\xi_{1}, x_{2}\right)=\mathcal{F}(f)\left(\xi_{1}, x_{2}\right)=\frac{1}{(2 \pi)^{\frac{1}{2}}} \int_{\mathbb{R}} f\left(x_{1}, x_{2}\right) e^{-i x_{1} \xi_{1}} \mathrm{~d} x_{1} .
$$

We denote by $\|\hat{f}\|_{L_{\xi_{1}}^{p} L_{x_{2}}^{q}}$ the norm $\left(\int_{\mathbb{R}}\left(\int_{0}^{\infty}\left|\hat{f}\left(\xi_{1}, x_{2}\right)\right|^{q} \mathrm{~d} x_{2}\right)^{p / q} \mathrm{~d} \xi_{1}\right)^{1 / p}$. For $j=0,1$, we set

$$
\begin{aligned}
\|f\|_{X_{B_{\nu}}^{(\mu, \rho)}} & =\sum_{k=0,1}\left(\left\|\varphi_{B}^{(\mu, \rho)} X_{2}^{\frac{k}{2}}\left\langle\xi_{1}\right\rangle^{2} \hat{f}\left(\xi_{1}, X_{2}\right)\right\|_{L_{\xi_{1}}^{2} L_{X_{2}}^{1+k}}+\left\|\varphi_{B}^{(\mu, \rho)} X_{2}^{1+\frac{k}{2}}\left\langle\xi_{1}\right\rangle \partial_{X_{2}} \hat{f}\left(\xi_{1}, X_{2}\right)\right\|_{L_{\xi_{1}}^{2} L_{X_{2}}^{1+k}}\right), \\
\|f\|_{X_{I B_{\nu}, j}^{(\mu, \rho)}} & =\sum_{k=0,1}\left(\left\|\varphi_{B}^{(\mu, \rho)} X_{2}^{\frac{k}{2}}\left\langle\xi_{1}\right\rangle^{j} \hat{f}\left(\xi_{1}, X_{2}\right)\right\|_{L_{\xi_{1}}^{2} L_{X_{2}}^{1+k}}+\left\|\varphi_{B}^{(\mu, \rho)} X_{2}^{j+\frac{k}{2}} \partial_{X_{2}}^{j} \hat{f}\left(\xi_{1}, X_{2}\right)\right\|_{L_{\xi_{1}}^{2} L_{X_{2}}^{1+k}}\right), \\
\|f\|_{X_{I I \nu, j}^{(\mu, \theta)}} & =\left\|\varphi_{I}^{(\mu, \theta)}\left\langle\xi_{1}\right\rangle^{j} \hat{f}\left(\xi_{1}, x_{2}\right)\right\|_{L_{\xi_{1}}^{2} L_{x_{2}}^{2}}+\left\|\varphi_{I}^{(\mu, \theta)} \partial_{2}^{j} \hat{f}\left(\xi_{1}, x_{2}\right)\right\|_{L_{\xi_{1}}^{2} L_{x_{2}}^{2}}+\left\|\varphi_{I}^{(0, \theta)} f\right\|_{L_{x}^{1}} .
\end{aligned}
$$

The spaces $X_{B_{\nu}}^{(\mu, \rho)}, X_{I B_{\nu}, j}^{(\mu, \rho)}, X_{I I_{\nu}, j}^{(\mu, \theta)}$, are then naturally defined as the subspaces of $L^{2}\left(\mathbb{R}_{+}^{2}\right)$ equipped with

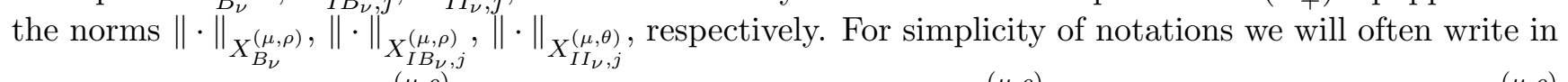
the abbreviated styles: $X_{B}^{(\mu, \rho)},\|\cdot\|_{X_{B}^{(\mu, \rho)}}$, and so on. The space $X_{B}^{(\mu, \rho)}$ will be applied for $w_{B}$, and $X_{I B, j}^{(\mu, \rho)}$ or $X_{I I, j}^{(\mu, \theta)}$ will be applied for $\omega_{I}$. It is useful to introduce the space for $\omega_{E}$ as follows.

$$
\begin{aligned}
\|f\|_{X_{E}^{(\mu, \theta)}} & =\left\|\varphi_{I}^{(\mu, \theta)}\left\langle\xi_{1}\right\rangle^{2} \hat{f}\left(\xi_{1}, x_{2}\right)\right\|_{L_{\xi_{1}}^{2} L_{x_{2}}^{2}}+\left\|\varphi_{I}^{(\mu, \theta)}\left\langle\xi_{1}\right\rangle \partial_{2} \hat{f}\left(\xi_{1}, x_{2}\right)\right\|_{L_{\xi_{1}}^{2} L_{x_{2}}^{2}}+\left\|\varphi_{I}^{(0, \theta)} f\right\|_{L_{x}^{1}}, \\
\|f\|_{Y_{E}} & =\|f\|_{W^{4,1}}+\|f\|_{W^{4,2}} .
\end{aligned}
$$

From (2.3) we may assume that $\omega_{E} \in L^{\infty}\left(0, T_{0} ; X_{E}^{\left(32 d_{E}, N\right)} \cap Y_{E}\right)$ for all $N \geq 0$. For convenience we will often use the notations

$$
X_{I B, 2}^{(\mu, \rho)}=X_{B}^{(\mu, \rho)}, \quad X_{I I, 2}^{(\mu, \theta)}=X_{E}^{(\mu, \theta)} .
$$

By the definition of the weights (2.10) - (2.11) the functions in $X_{I B, j}^{(\mu, \rho)}$ or $X_{I I, j}^{(\mu, \theta)}$ with $\mu>0$ are analytic in the tangential direction near the boundary. The form $\left(\mu-x_{2}\right)_{+}\left|\xi_{1}\right|$ represents how the analyticity is lost as the function leaves the boundary, and $\nu^{-1}\left(6 d_{E}-x_{2}\right)_{+}^{2}$ expresses the smallness exponentially in $\nu^{-1}$ near the boundary. These are in fact compatible with the heat equations, and thus, essential in our arguments; see Proposition 3.1. 


\subsection{Biot-Savart law}

In the vorticity formulation the velocity field is given by the Biot-Savart law $u=J(\omega)=\nabla^{\perp}\left(-\Delta_{D}\right)^{-1} \omega$. This section is devoted to give several estimates for $J(f)$ which are used in the latter sections.

Lemma 2.4 The following representations hold.

$$
\begin{aligned}
& \mathcal{F}\left(\partial_{1}\left(-\Delta_{D}\right)^{-1} f\right)\left(\xi_{1}, x_{2}\right)= \frac{1}{2} \frac{i \xi_{1}}{\left|\xi_{1}\right|}\left\{\int_{0}^{x_{2}} e^{-\left|\xi_{1}\right|\left(x_{2}-z_{2}\right)}\left(1-e^{-2\left|\xi_{1}\right| z_{2}}\right) \hat{f}\left(\xi_{1}, z_{2}\right) \mathrm{d} z_{2}\right. \\
&\left.+\int_{x_{2}}^{\infty} e^{-\left|\xi_{1}\right|\left(z_{2}-x_{2}\right)}\left(1-e^{-2\left|\xi_{1}\right| x_{2}}\right) \hat{f}\left(\xi_{1}, z_{2}\right) \mathrm{d} z_{2}\right\}, \\
& \mathcal{F}\left(\partial_{2}\left(-\Delta_{D}\right)^{-1} f\right)\left(\xi_{1}, x_{2}\right)=\frac{1}{2}\left\{-\int_{0}^{x_{2}} e^{-\left|\xi_{1}\right|\left(x_{2}-z_{2}\right)}\left(1-e^{-2\left|\xi_{1}\right| z_{2}}\right) \hat{f}\left(\xi_{1}, z_{2}\right) \mathrm{d} z_{2}\right. \\
&\left.+\int_{x_{2}}^{\infty} e^{-\left|\xi_{1}\right|\left(z_{2}-x_{2}\right)}\left(1+e^{-2\left|\xi_{1}\right| x_{2}}\right) \hat{f}\left(\xi_{1}, z_{2}\right) \mathrm{d} z_{2}\right\} .
\end{aligned}
$$

Proof. The required representations are obtained by solving the ODE: $\xi_{1}^{2} \hat{h}-\partial_{2}^{2} \hat{h}=\hat{f}$ in $x_{2}>0$ with the boundary condition $\hat{h}\left(\xi_{1}, 0\right)=\lim _{x_{2} \rightarrow \infty} \hat{h}\left(\xi_{1}, x_{2}\right)=0$. The details are omitted. This completes the proof.

Lemma 2.5 Let $k=0,1$ and $\rho>0$. Then it follows that

$$
\begin{aligned}
\|J(f)\|_{L^{4}} \lesssim\left\{\begin{array} { l } 
{ \| X _ { 2 } ^ { \frac { 1 } { 2 } } R _ { \nu } f \| _ { L ^ { 2 } } , } \\
{ \| f \| _ { L ^ { \frac { 4 } { 3 } } } , }
\end{array} \| J ( f ) \| _ { L ^ { \infty } } \lesssim \left\{\begin{array}{c}
\left\|R_{\nu} f\right\|_{X_{I B, 1}^{(0,0)}}, \\
\|f\|_{X_{I I, 1}^{(0,0)}}
\end{array}\right.\right. \\
d_{E}^{1+k}\left\|\chi_{\left\{x_{2} \geq 4 d_{E}\right\}} \nabla^{k} J(f)\right\|_{L^{4}}+d_{E}^{\frac{3}{2}+k}\left\|\chi_{\left\{x_{2} \geq 4 d_{E}\right\}} \nabla^{1+k} J(f)\right\|_{L^{2}} \lesssim\left(\frac{\nu}{\rho}\right)^{\frac{1}{2}}\left\|R_{\nu} f\right\|_{X_{I B, k}^{(0, \rho)}}, \\
\left\|\nabla^{k} J(f)\right\|_{L^{4}}+\left\|\nabla^{1+k} J(f)\right\|_{L^{2}} \lesssim\|f\|_{X_{I I, k}^{(0,0)}} .
\end{aligned}
$$

Proof. To prove (2.19) we use the representation

$$
\nabla\left(-\Delta_{D}\right)^{-1} f(x)=\frac{1}{2 \pi} \int_{\mathbb{R}_{+}^{2}}\left(\frac{x-y}{|x-y|^{2}}-\frac{x-y^{*}}{\left|x-y^{*}\right|^{2}}\right) f(y) \mathrm{d} y \quad y^{*}=\left(y_{1},-y_{2}\right) .
$$

Hence we have $|J(f)(x)| \lesssim \int_{\mathbb{R}_{+}^{2}} \frac{y_{2}}{|x-y|\left|x-y^{*}\right|}|f(y)| \mathrm{d} y \lesssim \int_{\mathbb{R}_{+}^{2}} \frac{y_{2}^{1 / 2}}{|x-y|^{3 / 2}}|f(y)| \mathrm{d} y$, which implies the estimate $\|J(f)\|_{L^{4}} \lesssim\left\|x_{2}^{1 / 2} f\right\|_{L^{2}}=\left\|X_{2}^{1 / 2} R_{\nu} f\right\|_{L^{2}}$ by the Hardy-Littlewood-Sobolev inequality. The other estimate $\|J(f)\|_{L^{4}} \lesssim\|f\|_{L^{4 / 3}}$ is well known. Next, from $\|J(f)\|_{L^{\infty}} \lesssim\|J(f)\|_{L^{4}}^{1 / 2}\|\nabla J(f)\|_{L^{4}}^{1 / 2} \lesssim\|f\|_{L^{4 / 3}}^{1 / 2}\|f\|_{L^{4}}^{1 / 2}$ we have $\|J(f)\|_{L^{\infty}} \lesssim\|f\|_{X_{I I, 1}^{(0,0)}}$ by the Sobolev embedding inequality and the interpolation inequality. When $f \in X_{I B, 1}^{(0,0)}$ we use the representation in Lemma 2.4. Then we have $\left|\mathcal{F}(J(f))\left(\xi_{1}, x_{2}\right)\right| \lesssim\left\|\hat{f}\left(\xi_{1}\right)\right\|_{L_{z_{2}}^{1}}$. This implies $|J(f)(x)| \lesssim\left\|\left\langle\xi_{1}\right\rangle \mathcal{F}(J(f))\left(\xi_{1}, x_{2}\right)\right\|_{L_{\xi_{1}}^{2}} \lesssim\left\|R_{\nu} f\right\|_{X_{I B, 1}^{(0,0)}}$. The proof of (2.19) is complete. To show (2.20) we decompose $f$ as $f=f \chi_{d_{E}}+f \chi_{d_{E}}^{c}$. If $x_{2} \geq 4 d_{E}$ then Lemma 2.4 leads to the inequality $\left|\mathcal{F}\left(J\left(f \chi_{d_{E}}\right)\right)\left(\xi_{1}, x_{2}\right)\right| \lesssim\left|\xi_{1}\right| e^{-\left|\xi_{1}\right| x_{2} / 2}\left\|z_{2} \hat{f}\left(\xi_{1}\right)\right\|_{L_{z_{2}}^{1}}$, i.e., $\left\|\chi_{\left\{x_{2} \geq 4 d_{E}\right\}} \mathcal{F}\left(J\left(f \chi_{d_{E}}\right)\right)\right\|_{L_{x_{2}}^{4} L_{\xi_{1}}^{4 / 3}} \lesssim d_{E}^{-1}\left\|x_{2} \hat{f}\right\|_{L_{\xi_{1}}^{2} L_{x_{2}}^{1}}$. Thus, combining the Hausdorff-Young inequality with the estimate $x_{2} e^{-\rho x_{2}^{2} / \nu} \lesssim(\nu / \rho)^{1 / 2}$, we arrive at $\left\|\chi_{\left\{x_{2} \geq 4 d_{E}\right\}} J\left(f \chi_{d_{E}}\right)\right\|_{L^{4}} \lesssim d_{E}^{-1}(\nu / \rho)^{1 / 2}\left\|R_{\nu} f\right\|_{X_{I B, 0}^{(0, \rho)}}$. Similarly, it is not difficult to see that $d_{E}^{2}\left\|\chi_{\left\{x_{2} \geq 4 d_{E}\right\}} \nabla J\left(f \chi_{d_{E}}\right)\right\|_{L^{4}} \lesssim\left(\frac{\nu}{\rho}\right)^{\frac{1}{2}}\left\|R_{\nu} f\right\|_{X_{I B, 1}^{(0, \rho)},} \quad d_{E}^{\frac{3}{2}+k}\left\|\chi_{\left\{x_{2} \geq 4 d_{E}\right\}} \nabla^{1+k} J\left(f \chi_{d_{E}}\right)\right\|_{L^{2}} \lesssim\left(\frac{\nu}{\rho}\right)^{\frac{1}{2}}\left\|R_{\nu} f\right\|_{X_{I B, k}^{(0, \rho)}}$. 
Next we see that $\left\|\nabla^{k} J\left(f \chi_{d_{E}}^{c}\right)\right\|_{L^{4}} \lesssim\left\|x_{2}^{1 / 2} \nabla^{k}\left(f \chi_{d_{E}}^{c}\right)\right\|_{L^{2}}$ and $\left\|\nabla^{1+k} J\left(f \chi_{d_{E}}^{c}\right)\right\|_{L^{2}} \lesssim\left\|\nabla^{k}\left(f \chi_{d_{E}}^{c}\right)\right\|_{L^{2}}$ for $k=$ 0,1 . Thus, since $d_{E} \in(0,1 / 2)$, it immediately follows that

$$
d_{E}^{1+k}\left\|\nabla^{1+k}\left(-\Delta_{D}\right)^{-1}\left(f \chi_{d_{E}}^{c}\right)\right\|_{L^{4}} \lesssim\left(\frac{\nu}{\rho}\right)^{\frac{1}{2}}\|f\|_{X_{I B, k}^{(0, \rho)}}, \quad d_{E}^{\frac{3}{2}+k}\left\|\nabla^{2+k}\left(-\Delta_{D}\right)^{-1}\left(f \chi_{d_{E}}^{c}\right)\right\|_{L^{2}} \lesssim\left(\frac{\nu}{\rho}\right)^{\frac{1}{2}}\|f\|_{X_{I B, k}^{(0, \rho)}} .
$$

The proof of (2.20) is complete. The estimate (2.21) with $k=0$ is easily obtained from the HardyLittlewood-Sobolev inequality and the Calderón-Zygmund inequality. For $k=1$ it suffices to note $\|\nabla J(f)\|_{L^{4}} \lesssim\|f\|_{L^{4}}$ and $\left\|\nabla^{2} J(f)\right\|_{L^{2}} \lesssim\|\nabla f\|_{L^{2}}+\left\|\nabla J\left(\partial_{1} f\right)\right\|_{L^{2}} \lesssim\|\nabla f\|_{L^{2}}$. This completes the proof.

Combining Lemma 2.5 with the Hölder inequality and the Sobolev embedding inequality, we get the following lemma, whose proof is omitted here.

Lemma 2.6 Let $1 \leq p \leq 4, k=0,1$, and $\rho>0$. Let $B(f, h)$ be the bilinear form in (2.2). Assume that supp $h \subset\left\{x \in \mathbb{R}_{+}^{2} \mid x_{2} \geq 4 d_{E}\right\}$. Then we have

$$
\begin{aligned}
\|B(f, h)\|_{L^{p}} \lesssim\left\{\begin{array}{l}
d_{E}^{-1}\left(\frac{\nu}{\rho}\right)^{\frac{1}{2}}\left\|R_{\nu} f\right\|_{X_{I B, 0}^{(0, \rho)}} \\
\|f\|_{X_{I I, 0}^{(0,0)}}
\end{array}\right\}\|h\|_{Y_{E}}, \\
\left\|\nabla^{1+k} B(f, h)\right\|_{L^{2}} \lesssim\left\{\begin{array}{l}
d_{E}^{-\frac{3}{2}-k}\left(\frac{\nu}{\rho}\right)^{\frac{1}{2}}\left\|R_{\nu} f\right\|_{X_{I B, k}^{(0, \rho)}} \\
\|f\|_{X_{I I, k}^{(0,0)}}
\end{array}\right\}\|h\|_{Y_{E}} .
\end{aligned}
$$

\section{Estimates for linear and bilinear mappings}

In this section we establish the estimates for various mappings that appear in the vorticity equations. When we deal with the bilinear forms the following elementary inequalities will be freely used.

$$
\left\|\left\langle\xi_{1}\right\rangle^{j} \hat{F}_{1} * \hat{F}_{2}\right\|_{L_{\xi_{1}}^{2}} \lesssim\left\|\left\langle\xi_{1}\right\rangle^{1-l(j)} \hat{F}_{1}\right\|_{L_{\xi_{1}}^{2}}\left\|\left\langle\xi_{1}\right\rangle^{j+l(j)} \hat{F}_{2}\right\|_{L_{\xi_{1}}^{2}}, \quad\left\|\left\langle\xi_{1}\right\rangle^{2} \hat{F}_{1} * \hat{F}_{2}\right\|_{L_{\xi_{1}}^{2}} \lesssim\left\|\left\langle\xi_{1}\right\rangle^{2} \hat{F}_{1}\right\|_{L_{\xi_{1}}^{2}}\left\|\left\langle\xi_{1}\right\rangle^{2} \hat{F}_{2}\right\|_{L_{\xi_{1}}^{2}} .
$$

Here $j=0,1$, and $l(j)$ is defined by $l(1)=0$ and $l(0) \in\{0,1\}$.

\subsection{Basic linear estimates}

First we prove that the function spaces defined in Section 2.3 are invariant in a sense under the action of the heat semigroup, which gives the validity of our choice of the weight functions in Section 2.3. The key observation is the following simple inequalities, which will be combined with the heat kernel.

$$
\begin{aligned}
& \left(\mu-\nu^{\frac{1}{2}} X_{2}\right)_{+}\left|\xi_{1}\right| \leq\left(\mu-\nu^{\frac{1}{2}} Y_{2}\right)_{+}\left|\xi_{1}\right|+\nu^{\frac{1}{2}}\left|X_{2}-Y_{2}\right|\left|\xi_{1}\right| \leq\left(\mu-\nu^{\frac{1}{2}} Y_{2}\right)_{+}\left|\xi_{1}\right|+\nu(t-s) \xi_{1}^{2}+\frac{\left|X_{2}-Y_{2}\right|^{2}}{4(t-s)} \\
& \left(\mu-x_{2}\right)_{+}\left|\xi_{1}\right| \leq\left(\mu-y_{2}\right)_{+}\left|\xi_{1}\right|+\left|x_{2}-x_{2}\right|\left|\xi_{1}\right| \leq\left(\mu-y_{2}\right)_{+}\left|\xi_{1}\right|+\nu(t-s) \xi_{1}^{2}+\frac{\left|x_{2}-y_{2}\right|^{2}}{4 \nu(t-s)}
\end{aligned}
$$

In Proposition 3.1 below we give the estimates for $e^{\nu(t-s) \Delta_{N}} f$. But it is clear from its proof that all estimates in Proposition 3.1 are valid even if the kernel of $e^{\nu(t-s) \Delta_{N}}$ is replaced by the two-dimensional Gaussian-type functions $g\left(c_{1} \nu(t-s), x_{1}-y_{1}\right) g\left(c_{2} \nu(t-s), x_{2}-y_{2}\right)$, where $g$ is the one-dimensional Gaussian and $c_{1}, c_{2}$ are suitable positive parameters. This fact will be used in the latter sections. 
Proposition 3.1 Let $k, l \in \mathbb{N} \cup\{0\}, m, n=0,1$, and $0 \leq \gamma \leq 1$. Assume that $0<s<t, 0<\mu^{\prime}<\mu$, $0<\rho^{\prime}<\rho \leq 2^{-4}$, and $0<\theta^{\prime}<\theta \leq 2^{-4}$. Then

$$
\begin{aligned}
&\left\|\varphi_{B}^{\left(\mu, \frac{\rho}{t}\right)}\left\langle\xi_{1}\right\rangle^{k+l} X_{2}^{\frac{m}{2}} \mathcal{F}\left(R_{\nu} e^{\nu(t-s) \Delta_{N}} R_{\frac{1}{\nu}} f\right)\right\|_{L_{\xi_{1}}^{2} L_{X_{2}}^{1+m}} \lesssim \frac{1}{(\nu(t-s))^{\frac{l}{2}}} \sum_{j=0}^{m}\left\|\varphi_{B}^{\left(\mu, \frac{\rho}{s}\right)}\left\langle\xi_{1}\right\rangle^{k} X_{2}^{\frac{j}{2}} \hat{f}\right\|_{L_{\xi_{1}}^{2} L_{X_{2}}^{1+j}}, \\
&\left\|\varphi_{B}^{\left(\mu^{\prime}, \frac{\rho^{\prime}}{t}\right)}\left\langle\xi_{1}\right\rangle^{k+n} X_{2}^{1-n+\frac{m}{2}} \partial_{X_{2}}^{1-n} \mathcal{F}\left(R_{\nu} e^{\nu(t-s) \Delta_{N}} R_{\frac{1}{\nu}} f\right)\right\|_{L_{\xi_{1}}^{2} L_{X_{2}}^{1+m}} \lesssim\left(\frac{1}{\left(\mu-\mu^{\prime}\right)^{n}}+\frac{s^{\frac{1}{2}}}{\mu^{\prime n}(t-s)^{\frac{1}{2}}\left(\rho-\rho^{\prime}\right)^{\frac{1}{2}}}\right) \\
&\left\|\varphi_{I}^{\left(\mu, \frac{\theta}{t}\right)}\left\langle\xi_{1}\right\rangle^{k+l} \mathcal{F}\left(e^{\nu(t-s) \Delta_{N}} f\right)\right\|_{L_{\xi_{1}}^{2} L_{x_{2}}^{2}} \\
& \qquad \frac{1}{(\nu(t-s))^{\frac{l}{2}}} \| \varphi_{I}^{\left(\mu, \frac{\theta}{s}\right)}\left\langle\varphi_{B}^{\left(\mu, \frac{\rho}{s}\right)}\left\langle\xi_{1}\right\rangle^{k} X_{2}^{\frac{j}{2}} \hat{f} \|_{L_{\xi_{1}}^{2} \|_{X_{2}}^{1+j}}, \quad\right. \text { (3.5) } \\
&\left\|\varphi_{\xi_{1} L_{x_{2}}^{2}}^{\left(\mu, \frac{\theta^{\prime}}{t}\right)}\left\langle\xi_{1}\right\rangle^{k+m} \partial_{2}^{1-m} \mathcal{F}\left(e^{\nu(t-s) \Delta_{N}} f \chi_{4 d_{E}}\right)\right\|_{L_{\xi_{1}}^{2} L_{x_{2}}^{2}} \\
& \lesssim \frac{s^{\frac{\gamma}{2}}\left\|\varphi_{I}^{\left(\mu, \frac{\theta}{s}\right)}\left\langle\xi_{1}\right\rangle^{k} \hat{f} \chi_{4 d_{E}}\right\|_{L_{\xi_{1}}^{2} L_{x_{2}}^{2}}}{\nu^{\frac{1-\gamma}{2}} d_{E}^{\gamma}(t-s)^{\frac{1}{2}}\left(\theta-\theta^{\prime}\right)^{\frac{\gamma}{2}}}
\end{aligned}
$$

Proof. Let $g\left(t, X_{2}\right)$ be the one-dimensional Gaussian, i.e., $g\left(t, X_{2}\right)=(4 \pi t)^{-1 / 2} \exp \left(-X_{2}^{2} /(4 t)\right)$ and set $g\left(t, X_{2}, Y_{2}\right)=g\left(t, X_{2}-Y_{2}\right)+g\left(t, X_{2}+Y_{2}\right)$. We observe that

$$
\mathcal{F}\left(R_{\nu} e^{\nu(t-s) \Delta_{N}} R_{\frac{1}{\nu}} f\right)\left(\xi_{1}, X_{2}\right)=e^{-\nu(t-s) \xi_{1}^{2}} \int_{0}^{\infty} g\left(t-s, X_{2}, Y_{2}\right) e^{-\frac{1}{4}\left(\mu-\nu^{\frac{1}{2}} Y_{2}\right)_{+}\left|\xi_{1}\right|}\left(\varphi_{B}^{(\mu, 0)} \hat{f}\right)\left(\xi_{1}, Y_{2}\right) \mathrm{d} Y_{2} .
$$

Then we combine (3.2) with $e^{-\nu(t-s) \xi_{1}^{2}} g\left(t-s, X_{2}, Y_{2}\right)$, which leads to

$$
\varphi_{B}^{\left(\mu, \frac{\rho}{t}\right)}\left|\mathcal{F}\left(R_{\nu} e^{\nu(t-s) \Delta_{N}} R_{\frac{1}{\nu}} f\right)\left(\xi_{1}, X_{2}\right)\right| \lesssim e^{-\frac{3}{4} \nu(t-s) \xi_{1}^{2}} e^{\frac{\rho}{t} X_{2}^{2}} g(2(t-s)) *\left(\varphi_{B}^{(\mu, 0)}\left|\hat{f}\left(\xi_{1}\right)\right|\right)\left(X_{2}\right) .
$$

Here we have written as $h_{1} * h_{2}\left(X_{2}\right)=\int_{\mathbb{R}_{+}^{2}} h_{1}\left(X_{2}-Y_{2}\right) h_{2}\left(Y_{2}\right) \mathrm{d} Y_{2}$ for simplicity. Now we apply the weighted Young inequality (7.1) to get

$$
\left\|\varphi_{B}^{\left(\mu, \frac{\rho}{t}\right)} X_{2}^{\frac{m}{2}} \mathcal{F}\left(R_{\nu} e^{\nu(t-s) \Delta_{N}} R_{\frac{1}{\nu}} f\right)\left(\xi_{1}\right)\right\|_{L_{X_{2}}^{1+m}} \lesssim e^{-\frac{3}{4} \nu(t-s) \xi_{1}^{2}} \sum_{j=0}^{m}\left\|\varphi_{B}^{\left(\mu, \frac{\rho}{s}\right)} X_{2}^{\frac{j}{2}} \hat{f}\left(\xi_{1}\right)\right\|_{L_{X_{2}}^{1+j}}
$$

Note that the case $m=1 \mathrm{in}(3.10)$ is confirmed by using $X_{2}^{1 / 2} g\left(t-s, X_{2}, Y_{2}\right) \lesssim\left((t-s)^{1 / 4}+Y_{2}^{1 / 2}\right) g(5(t-$ $\left.s) / 4, X_{2}-Y_{2}\right)$, since the factor $(t-s)^{1 / 4}$ is canceled after applying the $L^{2}-L^{1}$ estimate in (7.1). Est.(3.4) is obtained by taking the $L^{2}$ norm with respect to $\xi_{1}$ in (3.10). To prove (3.5) we observe that $\left|\xi_{1}\right| e^{-\frac{1}{4}\left(\mu-\nu^{1 / 2} Y_{2}\right)_{+}\left|\xi_{1}\right|} \lesssim\left(\mu-\mu^{\prime}\right)^{-1} e^{-\frac{1}{4}\left(\mu^{\prime}-\nu^{1 / 2} Y_{2}\right)_{+}\left|\xi_{1}\right|}$ when $0 \leq Y_{2} \leq \mu^{\prime} / \nu^{1 / 2}, 0<\mu^{\prime}<\mu$, while $\left|\xi_{1}\right| e^{-\frac{1}{4} \nu(t-s) \xi_{1}^{2}-\rho Y_{2}^{2} / s} \lesssim \mu^{\prime-1} s^{1 / 2}(t-s)^{-1 / 2}\left(\rho-\rho^{\prime}\right)^{-1 / 2} e^{-\rho^{\prime} Y_{2}^{2} / s}$ when $Y_{2} \geq \mu^{\prime} / \nu^{1 / 2}, 0<\rho^{\prime}<\rho$. Then the expression (3.8) yields, instead of (3.9),

$$
\begin{aligned}
& \varphi_{B}^{\left(\mu^{\prime}, \frac{\rho^{\prime}}{t}\right)}\left|\mathcal{F}\left(R_{\nu} e^{\nu(t-s) \Delta_{N}} R_{\frac{1}{\nu}} f\right)\left(\xi_{1}, X_{2}\right)\right| \\
\lesssim & \left(\frac{1}{\mu-\mu^{\prime}}+\frac{s^{\frac{1}{2}}}{\mu^{\prime}(t-s)^{\frac{1}{2}}\left(\rho-\rho^{\prime}\right)^{\frac{1}{2}}}\right) e^{-\frac{3}{4} \nu(t-s) \xi_{1}^{2}} \int_{\mathbb{R}_{+}} g\left(2(t-s), X_{2}-Y_{2}\right) e^{-\frac{\rho^{\prime}}{s} Y_{2}^{2}}\left|\left(\varphi_{B}^{\left(\mu, \frac{\rho}{s}\right)} \hat{f}\right)\left(\xi_{1}, Y_{2}\right)\right| \mathrm{d} Y_{2} .
\end{aligned}
$$


This shows (3.5) with $n=1$. Similarly, the case $n=0$ is obtained by the inequality

$$
\left|X_{2} \partial_{X_{2}} g\left(t-s, X_{2}, Y_{2}\right)\right| e^{-\frac{\rho}{s} Y_{2}^{2}} \lesssim\left(1+\frac{s^{\frac{1}{2}}}{(t-s)^{\frac{1}{2}}\left(\rho-\rho^{\prime}\right)^{\frac{1}{2}}}\right) g\left(\frac{5}{4}(t-s), X_{2}-Y_{2}\right) e^{-\frac{\rho^{\prime}}{s} Y_{2}^{2}} \quad \text { for } \quad X_{2}, Y_{2} \geq 0 .
$$

The details are omitted. The proof of (3.6) is similar to the one of (3.4). Indeed, (3.3) implies

$$
\varphi_{I}^{\left(\mu, \frac{\theta^{\prime}}{t}\right)}\left|\mathcal{F}\left(e^{\nu(t-s) \Delta_{N}} f\right)\left(\xi_{1}, x_{2}\right)\right| \lesssim e^{-\frac{3}{4} \nu(t-s) \xi_{1}^{2}+\frac{\theta^{\prime}}{\nu t}\left(6 d_{E}-x_{2}\right)_{+}^{2}} g(2 \nu(t-s)) *\left(\varphi_{I}^{(\mu, 0)}\left|\hat{f}\left(\xi_{1}\right)\right|\right)\left(x_{2}\right) .
$$

Then applying (7.2) to (3.11) yields (3.6). To prove (3.7) with $m=1$ we use (3.6) and get

$$
\left\|\varphi_{I}^{\left(\mu, \frac{\theta^{\prime}}{t}\right)}\left\langle\xi_{1}\right\rangle^{k+1} \mathcal{F}\left(e^{\nu(t-s) \Delta_{N}} f \chi_{4 d_{E}}\right)\right\|_{L_{\xi_{1}}^{2} L_{x_{2}}^{2}} \lesssim \frac{1}{\nu^{\frac{1}{2}}(t-s)^{\frac{1}{2}}}\left\|\varphi_{I}^{\left(\mu, \frac{\theta^{\prime}}{s}\right)}\left\langle\xi_{1}\right\rangle^{k} \hat{f} \chi_{4 d_{E}}\right\|_{L_{\xi_{1}}^{2} L_{x_{2}}^{2}} .
$$

Then (3.7) with $m=1$ follows from the inequality

$$
\varphi_{I}^{\left(\mu, \frac{\theta^{\prime}}{s}\right)}\left(\xi_{1}, x_{2}\right) \leq e^{-\frac{\theta-\theta^{\prime}}{\nu s} d_{E}^{2}} \varphi_{I}^{\left(\mu, \frac{\theta}{s}\right)}\left(\xi_{1}, x_{2}\right) \lesssim\left(\frac{\nu s}{d_{E}^{2}\left(\theta-\theta^{\prime}\right)}\right)^{\frac{\gamma}{2}} \varphi_{I}^{\left(\mu, \frac{\theta}{s}\right)}\left(\xi_{1}, x_{2}\right)
$$

for $0 \leq x_{2} \leq 5 d_{E}$ and $0 \leq \gamma \leq 1$. The case $m=0$ is proved in the same way. This completes the proof.

Remark 3.2 From (3.10) we also have

$$
\left\|\varphi_{B}^{\left(\mu, \frac{\rho}{t}\right)}\left\langle\xi_{1}\right\rangle^{k+l} X_{2}^{\frac{m}{2}} \mathcal{F}\left(R_{\nu} e^{\nu(t-s) \Delta_{N}} R_{\frac{1}{\nu}} f\right)\right\|_{L_{\xi_{1}}^{2} L_{X_{2}}^{1+m}} \lesssim \frac{1}{(\nu(t-s))^{\frac{1}{4}+\frac{l}{2}}} \sum_{j=0}^{m}\left\|\varphi_{B}^{\left(\mu, \frac{\rho}{s}\right)}\left\langle\xi_{1}\right\rangle^{k} X_{2}^{\frac{j}{2}} \hat{f}\right\|_{L_{\xi_{1}}^{\infty} L_{X_{2}}^{1+j}},
$$

and (7.2) yields

$$
\left\|\varphi_{I}^{\left(0, \frac{\theta}{t}\right)} e^{\nu(t-s) \Delta_{N}} f\right\|_{L^{1}} \lesssim\left\|\varphi_{I}^{\left(0, \frac{\theta}{s}\right)} f\right\|_{L^{1}}
$$

Moreover, by using $\mathcal{F}\left(R_{\nu} e^{\nu(t-s) \Delta_{N}}\left(h \mathcal{H}_{\left\{x_{2}=0\right\}}^{1}\right)\right)=e^{-\nu(t-s) \xi_{1}^{2}} g\left(t-s, X_{2}\right) \hat{h}\left(\xi_{1}\right)$, we get the estimate

$$
\left\|\varphi_{B}^{\left(\mu, \frac{\rho}{t}\right)}\left\langle\xi_{1}\right\rangle^{k+j} X_{2}^{l+\frac{m}{2}} \partial_{X_{2}}^{l} \mathcal{F}\left(R_{\nu} e^{\nu(t-s) \Delta_{N}}\left(h \mathcal{H}_{\left\{x_{2}=0\right\}}^{1}\right)\right)\right\|_{L_{\xi_{1}}^{2} L_{X_{2}}^{1+m}} \lesssim \frac{1}{(\nu(t-s))^{\frac{j}{2}}}\left\|e^{\mu\left|\xi_{1}\right|}\left\langle\xi_{1}\right\rangle^{k} \hat{h}\right\|_{L_{\xi_{1}}^{2}}
$$

Since the proofs are straightforward we omit the details here.

\subsection{Estimates for bilinear forms (I)}

In this section we establish the estimates of the bilinear forms appearing in the nonlinear terms of the vorticity equations. In order to estimate the boundary layer parts $\left(w_{B}\right.$ and $\left.w_{I B}\right)$ it is convenient to rewrite the bilinear forms in (2.2) in the rescaled variables:

$$
B^{(\nu)}(f, h)\left(x_{1}, X_{2}\right)=R_{\nu} B\left(R_{\frac{1}{\nu}} f, R_{\frac{1}{\nu}} h\right)\left(x_{1}, X_{2}\right), \quad N^{(\nu)}(f, h)\left(x_{1}\right)=J_{1}\left(R_{\frac{1}{\nu}} B^{(\nu)}(f, h)\right)\left(x_{1}, 0\right) .
$$

Motivated by the relation $B(f, h)=J(f) \cdot \nabla h=\nabla \cdot(h J(f))$, we also introduce the bilinear forms

$$
\begin{aligned}
D(f, h) & =\left(D_{1}(f, h), D_{2}(f, h)\right)=h J(f), \\
D^{(\nu)}(f, h) & =\left(D_{1}^{(\nu)}(f, h), D_{2}^{(\nu)}(f, h)\right)=\left(R_{\nu} D_{1}\left(R_{\frac{1}{\nu}} f, R_{\frac{1}{\nu}} h\right), \nu^{-\frac{1}{2}} R_{\nu} D_{2}\left(R_{\frac{1}{\nu}} f, R_{\frac{1}{\nu}} h\right)\right) .
\end{aligned}
$$

Note that $B^{(\nu)}(f, h)=\nabla_{X} \cdot D^{(\nu)}(f, h)$ holds, where $\nabla_{X}=\left(\partial_{x_{1}}, \partial_{X_{2}}\right)$. In the proof of next lemma we set

$$
\hat{f}_{(\mu, \rho, \theta)}\left(\xi_{1}, X_{2}\right)=\varphi_{B}^{(\mu, \rho)}\left(\xi_{1}, X_{2}\right) \varphi_{I}^{(0, \theta)}\left(\xi_{1}, \nu^{\frac{1}{2}} X_{2}\right) \hat{f}\left(\xi_{1}, X_{2}\right) \text {. }
$$


Lemma 3.3 Assume that $0<2^{-1}\left(\mu-\mu^{\prime}\right)<\mu^{\prime}<\mu<1,0<\rho^{\prime}<\rho \leq 2^{-4}$, and $0<s<1$. Let $j, k=0,1$, and let $l(1)=0$ and $l(0) \in\{0,1\}$. (i) For $D^{(\nu)}(f, h)$ we have

$$
\begin{aligned}
\left\|\varphi_{B}^{\left(\mu, \frac{\rho}{s}\right)} \xi_{1}^{j} X_{2}^{\frac{k}{2}} \mathcal{F}\left(D_{1}^{(\nu)}(f, h)\right)\right\|_{L_{\xi_{1}}^{2} L_{X_{2}}^{1+k}} \lesssim\left\{\begin{array}{c}
\|f\|_{X_{I B, 1-l(j)}^{(\mu, 0)}} \\
\left\|R_{\frac{1}{\nu}} f\right\|_{X_{I I, 1-l(j)}^{(\mu, 0)}}^{(\mu, j)}
\end{array}\right\}\|h\|_{X_{I B, j+l(j)}^{(\mu, \rho)}}, \\
\left\|\varphi_{B}^{\left(\mu, \frac{\rho^{\prime}}{s}\right)} \xi_{1}^{j} X_{2}^{\frac{k}{2}} \mathcal{F}\left(D_{2}^{(\nu)}(f, h)\right)\right\|_{L_{\xi_{1}}^{2} L_{X_{2}}^{1+k}} \lesssim \frac{s^{\frac{1}{2}}}{\left(\rho-\rho^{\prime}\right)^{\frac{1}{2}}}\left\{\begin{array}{c}
\|f\|_{X_{I B, 2}^{(\mu, 0)}} \\
\left\|R_{\frac{1}{\nu}} f\right\|_{X_{I I, 2}^{(\mu, 0)}}
\end{array}\right\}\|h\|_{X_{I B, j}^{\left(\mu, \frac{\rho}{s}\right)}}, \\
\left\|\varphi_{B}^{\left(\mu, \frac{\rho}{s}\right)} \xi_{1}^{j} X_{2}^{\frac{k}{2}} \mathcal{F}\left(D_{2}^{(\nu)}(f, h)\right)\right\|_{L_{\xi_{1}}^{2} L_{X_{2}}^{1+k}} \lesssim \frac{1}{\nu^{\frac{1}{2}}}\left\{\begin{array}{c}
\|f\|_{X_{I B, 1-l(j)}^{(\mu, 0)}} \\
\left\|R_{\frac{1}{\nu}} f\right\|_{X_{I I, 1-l(j)}^{(\mu, 0)}}
\end{array}\right\}\|h\|_{X_{I B, j+l(j)}^{\left(\mu, \frac{\rho}{s}\right)}} .
\end{aligned}
$$

(ii) Let $m=0,1$. For $B^{(\nu)}(f, h)$ we have

$$
\begin{aligned}
\left\|\varphi_{B}^{\left(\mu, \frac{\rho}{s}\right)} \xi_{1}^{j} X_{2}^{\frac{k}{2}} \mathcal{F}\left(B_{1}^{(\nu)}(f, h)\right)\right\|_{L_{\xi_{1}}^{2} L_{X_{2}}^{1+k}} \lesssim\left\{\begin{array}{c}
\|f\|_{X_{I B, 1-l(j)}^{(\mu, 0)}} \\
\left\|R_{\frac{1}{\nu}} f\right\|_{X_{I I, 1-l(j)}^{(\mu, 0)}}
\end{array}\right\}\|h\|_{X_{I B, 1+j+l(j)}^{\left(\mu, \frac{\rho}{s}\right)}}, \\
\left\|\varphi_{B}^{\left(\mu^{\prime}, \frac{\rho}{s}\right)} \xi_{1}^{j} X_{2}^{\frac{k}{2}} \mathcal{F}\left(B_{2}^{(\nu)}(f, h)\right)\right\|_{L_{\xi_{1}}^{2} L_{X_{2}}^{1+k}} \lesssim \frac{1}{\left(\mu-\mu^{\prime}\right)^{1-m}}\left\{\begin{array}{c}
\|f\|_{X_{I B, m+j+l(j)}^{(\mu, 0)}}\|f\|_{X_{I I, m+j+l(j)}^{(\mu, 0)}}
\end{array}\right\}\|h\|_{X_{I B, 2-l(j)}^{\left(\mu, \frac{\rho}{s}\right)}}, \\
\left\|\varphi_{B}^{\left(\mu, \frac{\rho}{s}\right)} X_{2}^{m+\frac{k}{2}} \mathcal{F}\left(B_{2}^{(\nu)}(f, h)\right)\right\|_{L_{\xi_{1}}^{\frac{2}{m}} L_{X_{2}}^{1+k}} \lesssim \nu^{-\frac{m}{2}}\left\{\begin{array}{c}
\|f\|_{X_{I B, 1}^{(\mu, 0)}} \\
\left\|R_{\frac{1}{\nu}} f\right\|_{X_{I I, 1}^{(\mu, 0)}}
\end{array}\right\}\|h\|_{X_{I B, 1}^{(\mu, \rho)},} .
\end{aligned}
$$

(iii) Let $i=0,1,2$. For $N^{(\nu)}(f, h)$ we have

$$
\begin{aligned}
\left\|e^{\frac{\mu^{\prime}}{4}\left|\xi_{1}\right|} \xi_{1}^{i} \mathcal{F}\left(N^{(\nu)}(f, h)\right)\right\|_{L_{\xi_{1}}^{2}} \lesssim \frac{1}{\mu-\mu^{\prime}}\left\{\begin{array}{c}
\|f\|_{X_{I B, 2-m(i)-n(i)}^{(\mu, 0)}} \\
\left\|R_{\frac{1}{\nu}} f\right\|_{X_{I I, 2-m(i)-n(i)}^{(\mu, 0)}}
\end{array}\right\}\left\{\begin{array}{l}
\|h\|_{X_{I B, i+(1-i) n(i)}^{(\mu, 0)}} \\
\left\|R_{\frac{1}{\nu}} h\right\|_{X_{I I, i+(1-i) n(i)}^{(\mu, 0)}}
\end{array}\right\}_{(3.24)} \\
\left\|e^{\frac{\mu}{4}\left|\xi_{1}\right|}\left|\xi_{1}\right|^{j-1} \mathcal{F}\left(N^{(\nu)}(f, h)\right)\right\|_{L_{\xi_{1}}^{2}} \lesssim\left\{\begin{array}{c}
\|f\|_{X_{I B, 1-l(j)}^{(\mu, 0)}} \\
\left\|R_{\frac{1}{\nu}} f\right\|_{X_{I I, 1-l(j)}^{(\mu, 0)}}
\end{array}\right\}\left\{\begin{array}{c}
\|h\|_{X_{I B, j+l(j)}^{(\mu, 0)}} \\
\left\|R_{\frac{1}{\nu}} h\right\|_{X_{I I, j+l(j)}^{(\mu, 0)}}
\end{array}\right\} .
\end{aligned}
$$

Here $m(i)=0$ if $i=1,2$ and $m(0)=1$, and $n(2)=0, n(1)=1$, and $n(0) \in\{0,1\}$.

Proof. (i) By Lemma 2.4 and (3.17) we have the explicit formula

$$
\begin{aligned}
\mathcal{F}\left(D_{1}^{(\nu)}(f, h)\right)\left(\xi_{1}, X_{2}\right)= & \frac{1}{2} \int_{\mathbb{R}}\left\{-\int_{0}^{X_{2}} e^{-\nu^{\frac{1}{2}}\left|\eta_{1}\right|\left(X_{2}-Z_{2}\right)}\left(1-e^{-2 \nu^{\frac{1}{2}}\left|\eta_{1}\right| Z_{2}}\right) \hat{f}\left(\eta_{1}, Z_{2}\right) \mathrm{d} Z_{2}\right. \\
& \left.+\int_{X_{2}}^{\infty} e^{-\nu^{\frac{1}{2}}\left|\eta_{1}\right|\left(Z_{2}-X_{2}\right)}\left(1+e^{-2 \nu^{\frac{1}{2}}\left|\eta_{1}\right| Z_{2}}\right) \hat{f}\left(\eta_{1}, Z_{2}\right) \mathrm{d} Z_{2}\right\} \hat{h}\left(\xi_{1}-\eta_{1}, X_{2}\right) \mathrm{d} \eta_{1} .
\end{aligned}
$$

Then we have from $\left|\left(\mu-\nu^{1 / 2} X_{2}\right)_{+}-\left(\mu-\nu^{1 / 2} Z_{2}\right)_{+}\right| \leq \nu^{1 / 2}\left|X_{2}-Z_{2}\right|$ and $\left|\xi_{1}\right| \leq\left|\eta_{1}\right|+\left|\eta_{1}-\xi_{1}\right|$,

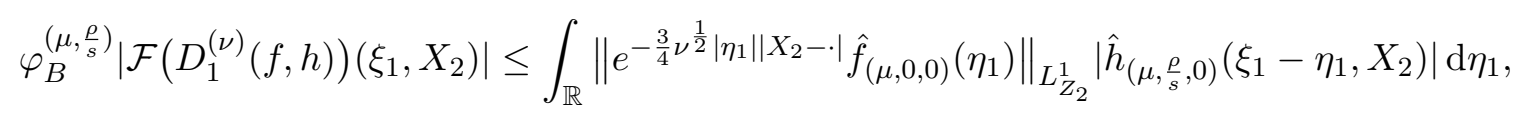


which implies for $j, k=0,1$,

$$
\begin{aligned}
& \varphi_{B}^{\left(\mu, \frac{\rho}{s}\right)}\left|\mathcal{F}\left(D_{1}^{(\nu)}(f, h)\right)\left(\xi_{1}, X_{2}\right)\right| \\
\leq & \int_{\mathbb{R}}\left\{\begin{array}{l}
\left\|\hat{f}_{(\mu, 0,0)}\left(\eta_{1}\right)\right\|_{L_{X_{2}}^{1}} \\
\chi_{\left\{\left|\eta_{1}\right| \leq 1\right\}}\|f\|_{L^{1}}+\frac{\chi_{\left\{\left|\eta_{1}\right| \geq 1\right\}}}{\nu^{\frac{1}{4}}\left|\eta_{1}\right|^{\frac{1}{2}}}\left\|\hat{f}_{(\mu, 0,0)}\left(\eta_{1}\right)\right\|_{L_{X_{2}}^{2}}
\end{array}\right\}\left|\hat{h}_{\left(\mu, \frac{\rho}{s}, 0\right)}\left(\xi_{1}-\eta_{1}, X_{2}\right)\right| \mathrm{d} \eta_{1},
\end{aligned}
$$

which gives (3.18) by the relation of the scaling $\nu^{-1 / 4}\|F\|_{L_{X_{2}}^{2}}=\left\|R_{1 / \nu} F\right\|_{L_{x_{2}}^{2}}$ and the inequality (3.1). The calculation as in (3.26) yields

$$
\begin{array}{r}
\varphi_{B}^{\left(\mu, \frac{\rho^{\prime}}{s}\right)}\left|\mathcal{F}\left(D_{2}^{(\nu)}(f, h)\right)\left(\xi_{1}, X_{2}\right)\right|=\varphi_{B}^{\left(\mu, \frac{\rho^{\prime}}{s}\right)} \mid-\frac{1}{2} \int_{\mathbb{R}} \frac{i \eta_{1}}{\nu^{\frac{1}{2}}\left|\eta_{1}\right|}\left\{\int_{0}^{X_{2}} e^{-\nu^{\frac{1}{2}}\left|\eta_{1}\right|\left(X_{2}-Z_{2}\right)}\left(1-e^{-2 \nu^{\frac{1}{2}}\left|\eta_{1}\right| Z_{2}}\right) \hat{f}\left(\eta_{1}, Z_{2}\right) \mathrm{d} Z_{2}\right. \\
\left.+\int_{X_{2}}^{\infty} e^{-\nu^{\frac{1}{2}}\left|\eta_{1}\right|\left(Z_{2}-X_{2}\right)}\left(1-e^{-2 \nu^{\frac{1}{2}}\left|\eta_{1}\right| X_{2}}\right) \hat{f}\left(\eta_{1}, Z_{2}\right) \mathrm{d} Z_{2}\right\} \hat{h}\left(\xi_{1}-\eta_{1}, X_{2}\right) \mathrm{d} \eta_{1} \mid \\
\lesssim X_{2} e^{-\frac{\rho-\rho^{\prime}}{s} X_{2}^{2}} \int_{\mathbb{R}}\left\|\eta_{1} e^{-\frac{3}{4} \nu^{\frac{1}{2}}\left|\eta_{1}\right|\left|X_{2}-\cdot\right|} \hat{f}_{(\mu, 0,0)}\left(\eta_{1}\right)\right\|_{L_{Z_{2}}^{1}\left|\hat{h}_{\left(\mu, \frac{\rho}{s}, 0\right)}\left(\xi_{1}-\eta_{1}, X_{2}\right)\right| \mathrm{d} \eta_{1} . \quad}
\end{array}
$$

Then it is not difficult to deduce (3.19). On the other hand, instead of (3.28), we also have

$$
\varphi_{B}^{\left(\mu, \frac{\rho}{s}\right)}\left|\mathcal{F}\left(D_{2}^{(\nu)}(f, h)\right)\left(\xi_{1}, X_{2}\right)\right| \lesssim \nu^{-\frac{1}{2}} \int_{\mathbb{R}}\left\|e^{-\frac{3}{4} \nu^{\frac{1}{2}}\left|\eta_{1}\right|\left|X_{2}-\cdot\right|} \hat{f}_{(\mu, 0,0)}\left(\eta_{1}\right)\right\|_{L_{Z_{2}}^{1}}\left|\hat{h}_{\left(\mu, \frac{\rho}{s}, 0\right)}\left(\xi_{1}-\eta_{1}, X_{2}\right)\right| \mathrm{d} \eta_{1} .
$$

Thus (3.20) follows by arguing as (3.27). (ii) As in the proof of (i), we observe that

$$
\begin{aligned}
& \varphi_{B}^{\left(\mu, \frac{\rho}{s}\right)}\left|\mathcal{F}\left(B_{1}^{(\nu)}(f, h)\right)\left(\xi_{1}, X_{2}\right)\right|=\varphi_{B}^{\left(\mu, \frac{\rho}{s}\right)} \mid \frac{1}{2} \int_{\mathbb{R}}\left\{-\int_{0}^{X_{2}} e^{-\nu^{\frac{1}{2}}\left|\eta_{1}\right|\left(X_{2}-Z_{2}\right)}\left(1-e^{-2 \nu^{\frac{1}{2}}\left|\eta_{1}\right| Z_{2}}\right) \hat{f}\left(\eta_{1}, Z_{2}\right) \mathrm{d} Z_{2}\right. \\
& \left.+\int_{X_{2}}^{\infty} e^{-\nu^{\frac{1}{2}}\left|\eta_{1}\right|\left(Z_{2}-X_{2}\right)}\left(1+e^{-2 \nu^{\frac{1}{2}}\left|\eta_{1}\right| X_{2}}\right) \hat{f}\left(\eta_{1}, Z_{2}\right) \mathrm{d} Z_{2}\right\} i\left(\xi_{1}-\eta_{1}\right) \hat{h}\left(\xi_{1}-\eta_{1}, X_{2}\right) \mathrm{d} \eta_{1} \mid \\
& \lesssim \int_{\mathbb{R}}\left\|e^{-\frac{3}{4} \nu^{\frac{1}{2}}\left|\eta_{1}\right|\left|X_{2}-\cdot\right|} \hat{f}_{(\mu, 0,0)}\left(\eta_{1}\right)\right\|_{L_{Z_{2}}^{1}}\left|\left(\xi_{1}-\eta_{1}\right) \hat{h}_{\left(\mu, \frac{\rho}{s}, 0\right)}\left(\xi_{1}-\eta_{1}, X_{2}\right)\right| \mathrm{d} \eta_{1},
\end{aligned}
$$

which gives (3.21) for $j, k=0,1$ by arguing as (3.27). As for $B_{2}^{(\nu)}(f, h)$, we see that if $\mu^{\prime} \geq \nu^{1 / 2} X_{2}$ then

$$
\begin{aligned}
& \varphi_{B}^{\left(\mu^{\prime}, \frac{\rho}{s}\right)}\left|\mathcal{F}\left(B_{2}^{(\nu)}(f, h)\right)\left(\xi_{1}, X_{2}\right)\right| \\
& =\varphi_{B}^{\left(\mu^{\prime}, \frac{\rho}{s}\right)} \mid-\frac{1}{2} \int_{\mathbb{R}} \frac{i \eta_{1}}{\left|\eta_{1}\right|}\left\{\int_{0}^{X_{2}} e^{-\nu^{\frac{1}{2}}\left|\eta_{1}\right|\left(X_{2}-Z_{2}\right)}\left(1-e^{-2 \nu^{\frac{1}{2}}\left|\eta_{1}\right| Z_{2}}\right) \hat{f}\left(\eta_{1}, Z_{2}\right) \mathrm{d} Z_{2}\right. \\
& \left.+\int_{X_{2}}^{\infty} e^{-\nu^{\frac{1}{2}}\left|\eta_{1}\right|\left(Z_{2}-X_{2}\right)}\left(1-e^{-2 \nu^{\frac{1}{2}}\left|\eta_{1}\right| X_{2}}\right) \hat{f}\left(\eta_{1}, Z_{2}\right) \mathrm{d} Z_{2}\right\} \nu^{-\frac{1}{2}} \partial_{X_{2}} \hat{h}\left(\xi_{1}-\eta_{1}, X_{2}\right) \mathrm{d} \eta_{1} \mid \\
& \lesssim \int_{\mathbb{R}}\left|\eta_{1}\right| e^{-\frac{1}{4}\left(\mu-\mu^{\prime}\right)\left(\left|\eta_{1}\right|+\left|\xi_{1}-\eta_{1}\right|\right)}\left\|e^{-\frac{3}{4} \nu^{\frac{1}{2}}\left|\eta_{1}\right|\left|X_{2}-\cdot\right|} \hat{f}_{(\mu, 0,0)}\left(\eta_{1}\right)\right\|_{L_{Z_{2}}^{1}}\left|X_{2} \partial_{X_{2}} \hat{h}_{\left(\mu, \frac{\rho}{s}, 0\right)}\left(\xi_{1}-\eta_{1}, X_{2}\right)\right| \mathrm{d} \eta_{1} \\
& \lesssim \frac{1}{\mu-\mu^{\prime}} \int_{\mathbb{R}} \frac{\left|\eta_{1}\right|}{\left|\eta_{1}\right|+\left|\xi_{1}-\eta_{1}\right|}\left\|e^{-\frac{3}{4} \nu^{\frac{1}{2}}\left|\eta_{1}\right|\left|X_{2}-\cdot\right|} \hat{f}_{(\mu, 0,0)}\left(\eta_{1}\right)\right\|_{L_{Z_{2}}^{1}}\left|X_{2} \partial_{X_{2}} \hat{h}_{\left(\mu, \frac{\rho}{s}, 0\right)}\left(\xi_{1}-\eta_{1}, X_{2}\right)\right| \mathrm{d} \eta_{1},
\end{aligned}
$$

and if $\mu^{\prime} \leq \nu^{1 / 2} X_{2}$ then

$$
\begin{aligned}
& \varphi_{B}^{\left(\mu^{\prime}, \frac{\rho}{s}\right)}\left|\mathcal{F}\left(B_{2}^{(\nu)}(f, h)\right)\left(\xi_{1}, X_{2}\right)\right| \lesssim \int_{\mathbb{R}}\left\|e^{-\nu^{\frac{1}{2}}\left|\eta_{1}\right|\left|X_{2}-\cdot\right|} \hat{f}\left(\eta_{1}\right)\right\|_{L_{Z_{2}}^{1}} \nu^{-\frac{1}{2}}\left|\partial_{X_{2}} \hat{h}_{\left(\mu, \frac{\rho}{s}, 0\right)}\left(\xi_{1}-\eta_{1}, X_{2}\right)\right| \mathrm{d} \eta_{1} \\
& \lesssim \frac{1}{\mu^{\prime}} \int_{\mathbb{R}}\left\|e^{-\nu^{\frac{1}{2}}\left|\eta_{1} \| X_{2}-\cdot\right|} \hat{f}\left(\eta_{1}\right)\right\|_{L_{Z_{2}}^{1}}\left|X_{2} \partial_{X_{2}} \hat{h}_{\left(\mu, \frac{\rho}{s}, 0\right)}\left(\xi_{1}-\eta_{1}, X_{2}\right)\right| \mathrm{d} \eta_{1} .
\end{aligned}
$$


Combining (3.31) and (3.32), we get (3.22) for $j, k=0,1$, and $m=0$. On the other hand, we also have

$$
\varphi_{B}^{\left(\mu, \frac{\rho}{s}\right)}\left|\mathcal{F}\left(B_{2}^{(\nu)}(f, h)\right)\left(\xi_{1}, X_{2}\right)\right| \lesssim \int_{\mathbb{R}}\left\|\eta_{1} e^{-\frac{3}{4} \nu^{\frac{1}{2}}\left|\eta_{1}\right|\left|X_{2}-\cdot\right|} \hat{f}_{(\mu, 0,0)}\left(\eta_{1}\right)\right\|_{L_{Z_{2}}^{1}}\left|X_{2} \partial_{X_{2}} \hat{h}_{\left(\mu, \frac{\rho}{s}, 0\right)}\left(\xi_{1}-\eta_{1}, X_{2}\right)\right| \mathrm{d} \eta_{1},
$$

which gives (3.22) with $m=1$. Est.(3.23) is proved in the same manner by using (3.33) for $m=0$, and

$$
\left|X_{2} \mathcal{F}\left(B_{2}^{(\nu)}(f, h)\right)\left(\xi_{1}, X_{2}\right)\right| \lesssim \nu^{-\frac{1}{2}} \int_{\mathbb{R}}\left\|e^{-\nu^{\frac{1}{2}}\left|\eta_{1}\right|\left|X_{2}-\cdot\right|} \hat{f}\left(\eta_{1}\right)\right\|_{L_{Z_{2}}^{1}}\left|X_{2} \partial_{X_{2}} \hat{h}\left(\xi_{1}-\eta_{1}, X_{2}\right)\right| \mathrm{d} \eta_{1} .
$$

for $m=1$. The details are omitted here. (iii) From the definition of $N^{(\nu)}(f, h)$ we have

$$
\mathcal{F}\left(N^{(\nu)}(f, h)\right)\left(\xi_{1}\right)=\int_{0}^{\infty} e^{-\nu^{\frac{1}{2}}\left|\xi_{1}\right| Y_{2}}\left(i \xi_{1}, \nu^{\frac{1}{2}}\left|\xi_{1}\right|\right) \cdot \mathcal{F}\left(D^{(\nu)}(f, h)\right)\left(\xi_{1}, Y_{2}\right) \mathrm{d} Y_{2} .
$$

This yields, from the arguments as in (3.26), (3.27), and (3.29), that

$$
\begin{aligned}
& \left|\mathcal{F}\left(N^{(\nu)}(f, h)\right)\left(\xi_{1}\right)\right| \lesssim\left|\xi_{1}\right| \int_{0}^{\infty} e^{-\nu^{\frac{1}{2}}\left|\xi_{1}\right| Y_{2}-\frac{1}{4}\left(\mu-\nu^{\frac{1}{2}} Y_{2}\right)_{+}\left|\xi_{1}\right|} \\
& \cdot \int_{\mathbb{R}}\left\{\begin{array}{l}
\left\|\hat{f}_{(\mu, 0,0)}\left(\eta_{1}\right)\right\|_{L_{X_{2}}^{1}} \\
\|f\|_{L^{1} \chi_{\left\{\left|\eta_{1}\right| \leq 1\right\}}}+\frac{\left\|\hat{f}_{(\mu, 0,0)}\left(\eta_{1}\right)\right\|_{L_{X_{2}}^{2}} \chi_{\left\{\left|\eta_{1}\right| \geq 1\right\}}}{\nu^{\frac{1}{4}}\left|\eta_{1}\right|^{\frac{1}{2}}}
\end{array}\right\}\left|\hat{h}_{(\mu, 0,0)}\left(\xi_{1}-\eta_{1}, Y_{2}\right)\right| \mathrm{d} \eta_{1} \mathrm{~d} Y_{2} \\
& \lesssim\left|\xi_{1}\right| e^{-\frac{1}{4} \mu^{\prime}\left|\xi_{1}\right|} \int_{\mathbb{R}}\left\{\begin{array}{l}
\left\|\hat{f}_{(\mu, 0,0)}\left(\eta_{1}\right)\right\|_{L_{X_{2}}^{1}} \\
\|f\|_{L^{1}} \chi_{\left\{\left|\eta_{1}\right| \leq 1\right\}}+\frac{\left\|\hat{f}_{(\mu, 0,0)}\left(\eta_{1}\right)\right\|_{L_{X_{2}}^{2}} \chi_{\left\{\left|\eta_{1}\right| \geq 1\right\}}}{\nu^{\frac{1}{4}}\left|\eta_{1}\right|^{\frac{1}{2}}}
\end{array}\right\} \\
& \cdot\left\|e^{-\frac{3}{4} \nu^{\frac{1}{2}}\left|\xi_{1}\right| Y_{2}}\left(e^{-\frac{1}{4}\left(\mu-\mu^{\prime}\right)\left|\xi_{1}\right|} \chi_{\left\{0 \leq Y_{2} \leq \frac{\mu^{\prime}}{\nu^{\frac{1}{2}}}\right\}}+\chi_{\left\{Y_{2} \geq \frac{\mu^{\prime}}{\nu \frac{1}{2}}\right\}}\right) \hat{h}_{(\mu, 0,0)}\left(\xi_{1}-\eta_{1}\right)\right\|_{L_{Y_{2}}^{1}} \mathrm{~d} \eta_{1} .
\end{aligned}
$$

Hence, we get (3.24) from (3.1) by performing two-ways estimates also for $h$ in the same manner as for $f$, and by using $\left|\xi_{1}\right| e^{-\nu^{1 / 2}\left|\xi_{1}\right| Y_{2} / 4} \lesssim\left(\mu-\mu^{\prime}\right)^{-1}$ when $Y_{2} \geq \mu^{\prime} \nu^{-1 / 2}$ and $2^{-1}\left(\mu-\mu^{\prime}\right)<\mu^{\prime}$. Est.(3.25) also directly follows from (3.35) (with $\mu^{\prime}=\mu$ ). The details are left to the reader. The proof is complete.

\subsection{Estimates for bilinear forms (II)}

Motivated by the integral equations stated in Section 2.2, we introduce the following bilinear forms.

$$
\begin{aligned}
& \Phi_{B, 1}^{(\nu)}[f, h](t)=-R_{\nu} e^{\nu t \Delta_{N}} R_{\frac{1}{\nu}} B^{(\nu)}(f, h), \quad \Phi_{B, 2}^{(\nu)}[f, h](t)=R_{\nu} e^{\nu t \Delta_{N}}\left(N^{(\nu)}(f, h) \mathcal{H}_{\left\{X_{2}=0\right\}}^{1}\right), \\
& \Psi_{1}^{(\nu)}[f, h](t)=-R_{\nu} \Gamma(\nu t) \star R_{\frac{1}{\nu}} B^{(\nu)}(f, h), \quad \Psi_{2}^{(\nu)}[f, h](t)=R_{\nu} \Gamma(\nu t) \star\left(N^{(\nu)}(f, h) \mathcal{H}_{\left\{X_{2}=0\right\}}^{1}\right), \\
& \Upsilon_{1}^{(\nu)}[f, h](t)=\nu R_{\nu} \int_{0}^{t} \Xi G(\nu(t-\tau)) \star R_{\frac{1}{\nu}} B^{(\nu)}(f, h) \mathrm{d} \tau, \\
& \Upsilon_{2}^{(\nu)}[f, h](t)=-\nu R_{\nu} \int_{0}^{t} \Xi G(\nu(t-\tau)) \star\left(N^{(\nu)}(f, h) \mathcal{H}_{\left\{X_{2}=0\right\}}^{1}\right) \mathrm{d} \tau .
\end{aligned}
$$

These are used for the boundary layer parts $w_{B}, w_{I B}$. Let $\chi_{l d_{E}}$ be the cut-off function defined by (1.5). For the interior part $w_{I I}$ we set $\Phi_{I}^{(\nu)}[f, h](t):=\sum_{i=1}^{3} \Phi_{I, i}^{(\nu)}[f, h](t)$, where

$$
\begin{aligned}
& \Phi_{I, 1}^{(\nu)}[f, h](t)=-e^{\nu t \Delta_{N}} B\left(f, \chi_{4 d_{E}} h\right), \quad \Phi_{I, 2}^{(\nu)}[f, h](t)=-e^{\nu t \Delta_{N}} B\left(f, \chi_{4 d_{E}}^{c} \chi_{8 d_{E}} h\right), \\
& \Phi_{I, 3}^{(\nu)}[f, h](t)=-e^{\nu t \Delta_{N}} B\left(f, \chi_{8 d_{E}}^{c} h\right) .
\end{aligned}
$$


The rest of this section is devoted to establish the estimates for these bilinear forms. The basic strategy is to combine Proposition 3.1 with Lemma 3.3, but we need to take into account which function spaces $f$ and $h$ belong to. In particular, when both $f$ and $h$ correspond with the remainder parts $w_{I B}$ or $w_{I I}$ the prefactor $\nu^{-1 / 2}$ is allowed in the estimates (e.g. see (3.37)). In oder to ensure the lower bound of the existence time $T$ in Theorem 1.1, the dependence on the parameters $d_{E}, t, s$ has to be examined carefully, which requires some detailed calculations. In this section we always assume that $0<\nu<1$ and $0<s<t<1$. We also remind (3.1) and the statements just before Proposition 3.1.

Lemma 3.4 Assume that $0<2^{-1}\left(\mu-\mu^{\prime}\right)<\mu^{\prime}<\mu<1$ and $0<\rho^{\prime}<\rho \leq 2^{-4}$. Let $l(1)=0$ and $l(0) \in\{0,1\}$. (i) Let $j=0,1,2$, and $m=0,1$. Then

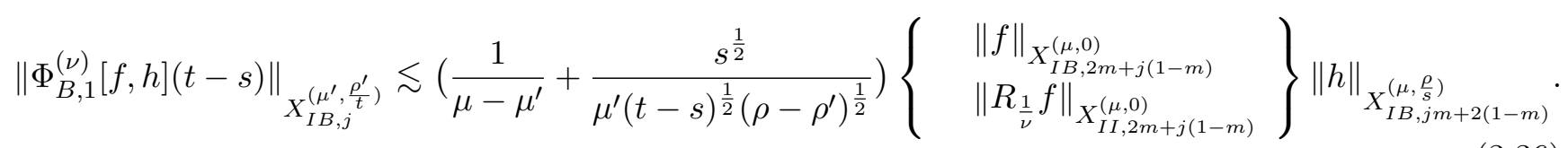

(ii) Let $j=0,1$. Then

$$
\left\|\Phi_{B, 1}^{(\nu)}[f, h](t-s)\right\|_{X_{I B, j}^{\left(\mu^{\prime}, \rho_{t}^{\prime}\right)}} \lesssim\left(\frac{1}{\nu^{\frac{1}{2}}(t-s)^{\frac{1}{2}}}+\frac{\nu^{\frac{1}{4}} s^{\frac{1}{2}}}{(t-s)^{\frac{1}{4}}\left(\rho-\rho^{\prime}\right)^{\frac{1}{2}}}\right)\left\{\begin{array}{c}
\|f\|_{X_{I B, 1-l(j)}^{(\mu, 0)}} \\
\left\|R_{\frac{1}{\nu}} f\right\|_{X_{I I, 1-l(j)}^{(\mu, 0)}}^{(\mu, 0)}
\end{array}\right\}\|h\|_{X_{I B, j+l(j)}^{\left(\mu, \frac{\rho}{s}\right)}} .
$$

Proof. In the proof below we sometimes write $\Phi_{B, 1}^{(\nu)}[f, h]$ instead of $\Phi_{B, 1}^{(\nu)}[f, h](t-s)$ for simplicity of notations. From the definitions of $B^{(\nu)}(f, h)$ and $D^{(\nu)}(f, h)$ we first decompose $\Phi_{B, 1}^{(\nu)}[f, h]$ as

$$
\Phi_{B, 1}^{(\nu)}[f, h]=-R_{\nu} e^{\nu(t-s) \Delta_{N}} R_{\frac{1}{\nu}} \partial_{1} D_{1}^{(\nu)}(f, h)-R_{\nu} e^{\nu(t-s) \Delta_{N}} R_{\frac{1}{\nu}} \partial_{X_{2}} D_{2}^{(\nu)}(f, h)=: \sum_{i=1,2} \Phi_{B, 1, i}^{(\nu)}[f, h] .
$$

Let $j=0,1$. Then we have from (3.5) and (3.18),

$$
\begin{aligned}
\left\|\varphi_{B}^{\left(\mu^{\prime}, \rho^{\prime}\right)} \xi_{1}^{j} X_{2}^{\frac{k}{2}} \mathcal{F}\left(\Phi_{B, 1,1}^{(\nu)}[f, h]\right)\right\|_{L_{\xi_{1}}^{2} L_{X_{2}}^{1+k}}=\left\|\varphi_{B}^{\left(\mu^{\prime}, \rho^{\prime}\right)} \xi_{1}^{j+1} X_{2}^{\frac{k}{2}} \mathcal{F}\left(R_{\nu} e^{\nu(t-s) \Delta_{N}} R_{\frac{1}{\nu}} D_{1}^{(\nu)}(f, h)\right)\right\|_{L_{\xi_{1}}^{2} L_{X_{2}}^{1+k}} \\
\lesssim\left(\frac{1}{\mu-\mu^{\prime}}+\frac{s^{\frac{1}{2}}}{\mu^{\prime}(t-s)^{\frac{1}{2}}\left(\rho-\rho^{\prime}\right)^{\frac{1}{2}}}\right)\left\{\begin{array}{c}
\|f\|_{X_{I B, 1-l(j)}^{(\mu, 0)}} \\
\left\|R_{\frac{1}{\nu}} f\right\|_{X_{I I, 1-l(j)}^{(\mu, 0)}}
\end{array}\right\}\|h\|_{X_{I B, j+l(j)}^{\left(\mu, \frac{\rho}{s}\right)}},
\end{aligned}
$$

and similarly from (3.4) and (3.18),

$$
\left\|\varphi_{B}^{\left(\mu^{\prime}, \frac{\rho^{\prime}}{t}\right)} \xi_{1}^{j} X_{2}^{\frac{k}{2}} \mathcal{F}\left(\Phi_{B, 1,1}^{(\nu)}[f, h]\right)\right\|_{L_{\xi_{1}}^{2} L_{X_{2}}^{1+k}} \lesssim \frac{1}{\nu^{\frac{1}{2}}(t-s)^{\frac{1}{2}}}\left\{\begin{array}{l}
\|f\|_{X_{I B, 1-l(j)}^{(\mu, 0)}} \\
\left\|R_{\frac{1}{\nu}} f\right\|_{X_{I I, 1-l(j)}^{(\mu, 0)}}
\end{array}\right\}\|h\|_{X_{I B, j+l(j)}^{\left(\mu, \frac{\rho}{s}\right)}} .
$$

Set $g^{*}\left(t, X_{2}, Y_{2}\right)=g\left(t, X_{2}-Y_{2}\right)-g\left(t, X_{2}+Y_{2}\right)$. For $\Phi_{B, 1,2}^{(\nu)}[f, h]$ we observe that

$$
\left|\mathcal{F}\left(\Phi_{B, 1,2}^{(\nu)}[f, h](t-s)\right)\left(\xi_{1}, X_{2}\right)\right|=\left|\int_{0}^{\infty} e^{-\nu(t-s) \xi_{1}^{2}} \partial_{X_{2}} g^{*}\left(t-s, X_{2}, Y_{2}\right) \mathcal{F}\left(D_{2}^{(\nu)}(f, h)\right)\left(\xi_{1}, Y_{2}\right) \mathrm{d} Y_{2}\right| .
$$

Hence, as in the proof of (3.4), one can verify with the aid of (3.19) that

$$
\left\|\varphi_{B}^{\left(\mu^{\prime}, \frac{\rho^{\prime}}{t}\right)} \xi_{1}^{j} X_{2}^{\frac{k}{2}} \mathcal{F}\left(\Phi_{B, 1,2}^{(\nu)}[f, h]\right)\right\|_{L_{\xi_{1}}^{2} L_{X_{2}}^{1+k}} \lesssim \frac{s^{\frac{1}{2}}}{(t-s)^{\frac{1}{2}}\left(\rho-\rho^{\prime}\right)^{\frac{1}{2}}}\left\{\begin{array}{l}
\|f\|_{X_{I B, 2}^{(\mu, 0)}} \\
\left\|R_{\frac{1}{\nu}} f\right\|_{X_{I I, 2}^{(\mu, 0)}}^{(\mu)}
\end{array}\right\}\|h\|_{X_{I B, j}^{\left(\mu, \frac{\rho}{s}\right)}} .
$$


Similarly, we have from (3.20),

$$
\left\|\varphi_{B}^{\left(\mu^{\prime}, \frac{\rho^{\prime}}{t}\right)} \xi_{1}^{j} X_{2}^{\frac{k}{2}} \mathcal{F}\left(\Phi_{B, 1,2}^{(\nu)}[f, h]\right)\right\|_{L_{\xi_{1}}^{2} L_{X_{2}}^{1+k}} \lesssim \frac{1}{\nu^{\frac{1}{2}}(t-s)^{\frac{1}{2}}}\left\{\begin{array}{l}
\|f\|_{X_{I B, 1-l(j)}^{(\mu, 0)}} \\
\left\|R_{\frac{1}{\nu}} f\right\|_{X_{I I, 1-l(j)}^{(\mu, 0)}}
\end{array}\right\}\|h\|_{X_{I B, j+l(j)}^{\left(\mu, \frac{\rho}{s}\right)}} .
$$

Collecting above estimates, so far we have shown that for $j, k=0,1$,

$$
\begin{aligned}
& \left\|\varphi_{B}^{\left(\mu^{\prime}, \frac{\rho^{\prime}}{t}\right)} \xi_{1}^{j} X_{2}^{\frac{k}{2}} \mathcal{F}\left(\Phi_{B, 1}^{(\nu)}[f, h]\right)\right\|_{L_{\xi_{1}}^{2} L_{X_{2}}^{1+k}} \lesssim\left(\frac{1}{\mu-\mu^{\prime}}+\frac{s^{\frac{1}{2}}}{\mu^{\prime}(t-s)^{\frac{1}{2}}\left(\rho-\rho^{\prime}\right)^{\frac{1}{2}}}\right)\left\{\begin{array}{c}
\|f\|_{X_{I B, 2}^{(\mu, 0)}} \\
\left\|R_{\frac{1}{\nu}} f\right\|_{X_{I I, 2}^{(\mu, 0)}}^{(\mu, j}
\end{array}\right\}\|h\|_{X_{I B, j}^{\left(\mu, \frac{\rho}{s}\right)}} \\
& \left\|\varphi_{B}^{\left(\mu^{\prime}, \frac{\rho^{\prime}}{t}\right)} \xi_{1}^{j} X_{2}^{\frac{k}{2}} \mathcal{F}\left(\Phi_{B, 1}^{(\nu)}[f, h]\right)\right\|_{L_{\xi_{1}}^{2} L_{X_{2}}^{1+k}} \lesssim \frac{1}{\nu^{\frac{1}{2}}(t-s)^{\frac{1}{2}}}\left\{\begin{array}{c}
\|f\|_{X_{I B, 1-l(j)}^{(\mu, 0)}} \\
\left\|R_{\frac{1}{\nu}} f\right\|_{X_{I I, 1-l(j)}^{(\mu, 0)}}^{(3.452)}
\end{array}\right\}\|h\|_{X_{I B, j+l(j)}^{\left(\mu, \frac{\rho}{s}\right)}} .
\end{aligned}
$$

In particular, (3.36) with $j=0, m=1$, and (3.37) with $j=0$ have been proved. To estimate the other norms we set $\Phi_{B, 1,1^{\prime}}^{(\nu)}[f, h](t-s)=-R_{\nu} e^{\nu(t-s) \Delta_{N}} R_{1 / \nu} B_{1}^{(\nu)}(f, h)$ and $\Phi_{B, 1,2^{\prime}}^{(\nu)}[f, h](t-s)=$ $-R_{\nu} e^{\nu(t-s) \Delta_{N}} R_{1 / \nu} B_{2}^{(\nu)}(f, h)$, which gives $\Phi_{B, 1}^{(\nu)}[f, h]=\sum_{i=1,2} \Phi_{B, 1, i^{\prime}}^{(\nu)}[f, h]$. Then (3.5), (3.21), and (3.22) imply

$$
\left\|\varphi_{B}^{\left(\mu^{\prime}, \frac{\rho^{\prime}}{t}\right)} \xi_{1}^{2} X_{2}^{\frac{k}{2}} \mathcal{F}\left(\Phi_{B, 1}^{(\nu)}[f, h]\right)\right\|_{L_{\xi_{1}}^{2} L_{X_{2}}^{1+k}} \lesssim\left(\frac{1}{\mu-\mu^{\prime}}+\frac{s^{\frac{1}{2}}}{\mu^{\prime}(t-s)^{\frac{1}{2}}\left(\rho-\rho^{\prime}\right)^{\frac{1}{2}}}\right)\left\{\begin{array}{l}
\|f\|_{X_{I B, 2}^{(\mu, 0)}} \\
\left\|R_{\frac{1}{\nu}} f\right\|_{X_{I I, 2}^{(\mu, 0)}}
\end{array}\right\}\|h\|_{X_{I B, 2}^{\left(\mu, \frac{\rho}{s}\right)}} .
$$

Moreover, (3.4), (3.5), and (3.21) imply that, by setting $S_{\tilde{j}, k, \tilde{k}}\left(\xi_{1}, X_{2}\right)=\xi_{1}^{\tilde{j}} X_{2}^{\tilde{k}+k / 2} \partial_{X_{2}}^{\tilde{k}}$ for $\tilde{j}, k, \tilde{k}=0,1$,

$$
\left\|\varphi_{B}^{\left(\mu^{\prime}, \frac{\rho^{\prime}}{t}\right)} S_{\tilde{j}, k, \tilde{k}} \mathcal{F}\left(\Phi_{B, 1,1^{\prime}}^{(\nu)}[f, h]\right)\right\|_{L_{\xi_{1}}^{2} L_{X_{2}}^{1+k}} \lesssim\left(1+\frac{s^{\frac{\tilde{k}}{2}}}{(t-s)^{\frac{\tilde{k}}{2}}\left(\rho-\rho^{\prime}\right)^{\frac{\tilde{k}}{2}}}\right)\left\{\begin{array}{c}
\|f\|_{X_{I B, 1-l(\tilde{j})}^{(\mu, 0)}} \\
\left\|R_{\frac{1}{\nu}} f\right\|_{X_{I I, 1-l(\tilde{j})}^{(\mu, 0)}}
\end{array}\right\}\|h\|_{X_{I B, 1+\tilde{j}+l(\tilde{j})}^{(\mu, \rho)}} .
$$

As for $\Phi_{B, 1,2^{\prime}}^{(\nu)}[f, h]$, we combine (3.22) with (3.4) if $\tilde{k}=0$ and with (3.5) if $\tilde{k}=1$ to obtain

$$
\left\|\varphi_{B}^{\left(\mu^{\prime}, \frac{\rho^{\prime}}{t}\right)} S_{\tilde{j}, k, \tilde{k}} \mathcal{F}\left(\Phi_{B, 1,2^{\prime}}^{(\nu)}[f, h]\right)\right\|_{L_{\xi_{1}}^{2} L_{X_{2}}^{1+k}} \lesssim\left(\frac{1}{\mu-\mu^{\prime}}+\frac{s^{\frac{1}{2}}}{\mu^{\prime}(t-s)^{\frac{1}{2}}\left(\rho-\rho^{\prime}\right)^{\frac{1}{2}}}\right)\left\{\begin{array}{c}
\|f\|_{X_{I B, \tau(\tilde{k}, \tilde{j})}^{(\mu, 0)}} \\
\left\|R_{\frac{1}{\nu}} f\right\|_{X_{I I, \tau(\tilde{k}, \tilde{j})}^{(\mu, 0)}}
\end{array}\right\}\|h\|_{X_{I B, 2-l(\tilde{j})}^{\left(\mu, \frac{\rho}{s}\right)}}
$$

for $\tau(\tilde{k}, \tilde{j})=\tilde{k}+\tilde{j}+l(\tilde{j})$. Collecting (3.42), (3.44), (3.45), and (3.46), we arrive at (3.36). So it remains to prove (3.37) for $j=1$, but in view of (3.43), it suffices to estimate $\partial_{X_{2}} \mathcal{F}\left(\Phi_{B, 1, i^{\prime}}^{(\nu)}[f, h]\right)$ for each $i=1,2$. First let us consider $\Phi_{B, 1,2^{\prime}}^{(\nu)}[f, h]$. Note that $\left|X_{2} \partial_{X_{2}} g\left(t-s, X_{2}, Y_{2}\right)\right| \lesssim\left(Y_{2}(t-s)^{-1 / 2}+1\right) g\left(5(t-s) / 4, X_{2}-Y_{2}\right)$ holds. Then we appeal to the estimate of the form (3.4) and (3.23) with $m=1$, and to (3.12) and (3.23) with $m=0$, which gives

$$
\sum_{k=0,1}\left\|\varphi_{B}^{\left(\mu^{\prime}, \frac{\rho^{\prime}}{t}\right)} X_{2}^{1+\frac{k}{2}} \partial_{X_{2}} \mathcal{F}\left(\Phi_{B, 1,2^{\prime}}^{(\nu)}[f, h]\right)\right\|_{L_{\xi_{1}}^{2} L_{X_{2}}^{1+k}} \lesssim \frac{1}{\nu^{\frac{1}{2}}(t-s)^{\frac{1}{2}}}\left\{\begin{array}{l}
\|f\|_{X_{I B, 1}^{(\mu, 0)}} \\
\left\|R_{\frac{1}{\nu}} f\right\|_{X_{I I, 1}^{(\mu, 0)}}
\end{array}\right\}\|h\|_{X_{I B, 1}^{\left(\mu, \frac{\rho}{s}\right)},},
$$


as desired. As for $\Phi_{B, 1,1^{\prime}}^{(\nu)}[f, h]$, we have from the integration by parts,

$$
\begin{aligned}
X_{2} \partial_{X_{2}} \mathcal{F}\left(\Phi_{B, 1,1^{\prime}}^{(\nu)}[f, h](t-s)\right)\left(\xi_{1}, X_{2}\right) & I_{0}^{\infty} e^{-\nu(t-s) \xi_{1}^{2}}\left(X_{2}-Y_{2}\right) \partial_{X_{2}} g\left(t-s, X_{2}, Y_{2}\right) \mathcal{F}\left(B_{1}^{(\nu)}(f, h)\right)\left(\xi_{1}, Y_{2}\right) \mathrm{d} Y_{2} \\
- & \int_{0}^{\infty} e^{-\nu(t-s) \xi_{1}^{2}} g^{*}\left(t-s, X_{2}, Y_{2}\right) \mathcal{F}\left(B_{1}^{(\nu)}(f, h)\right)\left(\xi_{1}, Y_{2}\right) \mathrm{d} Y_{2} \\
& -\int_{0}^{\infty} e^{-\nu(t-s) \xi_{1}^{2}} g^{*}\left(t-s, X_{2}, Y_{2}\right) Y_{2} \partial_{Y_{2}} \mathcal{F}\left(B_{1}^{(\nu)}(f, h)\right)\left(\xi_{1}, Y_{2}\right) \mathrm{d} Y_{2} .
\end{aligned}
$$

From the proof of (3.4) and (3.21) we see $\sum_{i=1}^{2}\left\|\varphi_{B}^{\left(\mu^{\prime}, \rho^{\prime} / t\right)} X_{2}^{k / 2} I_{i}\right\|_{L_{\xi_{1}}^{2} L_{X_{2}}^{1+k}} \lesssim\left\{\begin{array}{l}\|f\|_{X_{I B, 1}^{(\mu, 0)}} \\ \left\|R_{1 / \nu}\right\|_{X_{I I, 1}^{(\mu, 0)}}\end{array}\right\}\|h\|_{X_{I B, 1}^{(\mu, \rho / s)}}$. So it remains to estimate $I_{3}$. Set $I I_{3,1}=Y_{2} \partial_{Y_{2}} \mathcal{F}\left(B_{1}^{(\nu)}(f, h)\right)-\mathcal{F}\left(B_{1}^{(\nu)}\left(f, Y_{2} \partial_{Y_{2}} h\right)\right)$ and decompose $I_{3}$ into $I_{3,1}=-\int_{0}^{\infty} e^{-\nu(t-s) \xi_{1}^{2}} g^{*}\left(t-s, X_{2}, \cdot\right) I I_{3,1} \mathrm{~d} Y_{2}$ and $I_{3,2}=I_{3}-I_{3,1}$. The term $I I_{3,1}$ is expressed as

$$
\begin{gathered}
I I_{3,1}\left(\xi_{1}, Y_{2}\right)=\frac{1}{2} \int_{\mathbb{R}}\left(-2 Y_{2} \hat{f}\left(\eta_{1}, Y_{2}\right)+\nu^{\frac{1}{2}}\left|\eta_{1}\right| Y_{2}\left\{\int_{0}^{Y_{2}} e^{-\nu^{\frac{1}{2}}\left|\eta_{1}\right|\left(Y_{2}-Z_{2}\right)}\left(1-e^{-2 \nu^{\frac{1}{2}}\left|\eta_{1}\right| Z_{2}}\right) \hat{f}\left(\eta_{1}, Z_{2}\right) \mathrm{d} Z_{2}\right.\right. \\
\left.\left.+\int_{Y_{2}}^{\infty} e^{-\nu^{\frac{1}{2}}\left|\eta_{1}\right|\left(Z_{2}-Y_{2}\right)}\left(1-e^{-2 \nu^{\frac{1}{2}}\left|\eta_{1}\right| Y_{2}}\right) \hat{f}\left(\eta_{1}, Z_{2}\right) \mathrm{d} Z_{2}\right\}\right) i\left(\xi_{1}-\eta_{1}\right) \hat{h}\left(\xi_{1}-\eta_{1}, Y_{2}\right) \mathrm{d} \eta_{1} .
\end{gathered}
$$

Hence, by writing $\|\hat{F}\|_{L_{X_{2}}^{p}}$ instead of $\left\|\hat{F}\left(\eta_{1}\right)\right\|_{L_{X_{2}}^{p}}$ for simplicity of notations, we see

$$
\begin{aligned}
& \varphi_{B}^{\left(\mu^{\prime}, \frac{\rho^{\prime}}{s}\right)}\left|I I_{3,1}\left(\xi_{1}, Y_{2}\right)\right|
\end{aligned}
$$

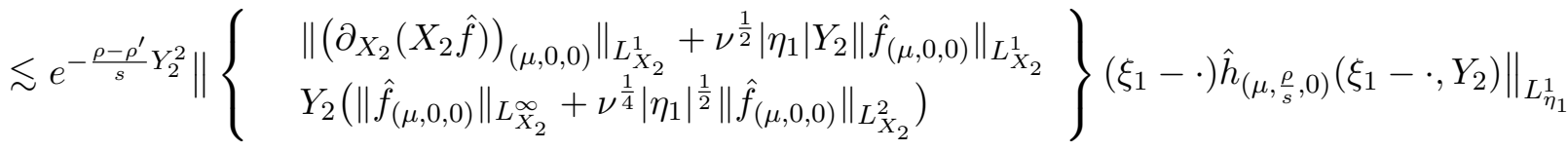

$$
\begin{aligned}
& \lesssim\left\|\left\{\begin{array}{c}
\left\|\left(\partial_{X_{2}}\left(X_{2} \hat{f}\right)\right)_{(\mu, 0,0)}\right\|_{L_{X_{2}}^{1}}+\frac{s^{\frac{1}{2}} \nu^{\frac{1}{2}}\left|\eta_{1}\right|}{\left(\rho-\rho^{\prime}\right)^{\frac{1}{2}}}\left\|\hat{f}_{(\mu, 0,0)}\right\|_{L_{X_{2}}^{1}} \\
\frac{s^{\frac{1}{2}}}{\left(\rho-\rho^{\prime}\right)^{\frac{1}{2}}}\left(\left\|\hat{f}_{(\mu, 0,0)}\right\|_{L_{X_{2}}^{\infty}}+\nu^{\frac{1}{4}}\left|\eta_{1}\right|^{\frac{1}{2}}\left\|\hat{f}_{(\mu, 0,0)}\right\|_{L_{X_{2}}^{2}}\right)
\end{array}\right\}\left(\xi_{1}-\cdot\right) \hat{h}_{\left(\mu, \frac{\rho}{s}, 0\right)}\left(\xi_{1}-\cdot, Y_{2}\right)\right\|_{L_{\eta_{1}}^{1}} .
\end{aligned}
$$

Then, using $\left\|\left(\partial_{X_{2}}\left(X_{2} \hat{f}\right)\right)_{(\mu, 0,0)}\right\|_{L_{\xi_{1}}^{2} L_{X_{2}}^{1}} \lesssim\|f\|_{X_{I B, 1}^{(\mu, 0)}}$ and $\left\|\hat{f}_{(\mu, 0,0)}\right\|_{L_{\xi_{1}}^{2} L_{X_{2}}^{\infty}} \lesssim \nu^{1 / 2}\left\|R_{1 / \nu} f\right\|_{X_{I I, 1}^{(\mu, 0)}}$ together with the estimate of the type (3.12) yields

$$
\left\|\varphi_{B}^{\left(\mu^{\prime}, \frac{\rho^{\prime}}{t}\right)} I_{3,1}\right\|_{L_{\xi_{1}}^{2} L_{X_{2}}^{1}} \lesssim\left(\frac{1}{\nu^{\frac{1}{4}}(t-s)^{\frac{1}{4}}}+\frac{\nu^{\frac{1}{4}} s^{\frac{1}{2}}}{(t-s)^{\frac{1}{4}}\left(\rho-\rho^{\prime}\right)^{\frac{1}{2}}}\right)\left\{\begin{array}{l}
\|f\|_{X_{I B, 1}^{(\mu, 0)}} \\
\left\|R_{\frac{1}{\nu}} f\right\|_{X_{I I, 1}^{(\mu, 0)}}
\end{array}\right\}\|h\|_{X_{I B, 1}^{\left(\mu, \frac{\rho}{s}\right)}} .
$$

On the other hand, the term $\mathcal{F}\left(B_{1}^{(\nu)}\left(f, Y_{2} \partial_{Y_{2}} h\right)\right)$ is estimated as

$$
\begin{aligned}
& \varphi_{B}^{\left(\mu^{\prime}, \frac{\rho^{\prime}}{s}\right)}\left|\mathcal{F}\left(B_{1}^{(\nu)}\left(f, Y_{2} \partial_{Y_{2}} h\right)\right)\left(\xi_{1}, Y_{2}\right)\right| \\
\lesssim & \left\|\left\{\begin{array}{l}
\left(\left|\eta_{1}\right|+\left|\xi_{1}\right|\right)\left\|\hat{f}_{(\mu, 0,0)}\right\|_{L_{X_{2}}^{1}} \\
\frac{\left|\eta_{1}\right|^{\frac{1}{2}}}{\nu^{\frac{1}{4}}}\left\|\hat{f}_{(\mu, 0,0)}\right\|_{L_{X_{2}}^{2}}+\left|\xi_{1}\right|\left(\chi_{\left\{\left|\eta_{1}\right| \leq 1\right\}}\|f\|_{L_{x}^{1}}+\frac{\chi_{\left\{\left|\eta_{1}\right| \geq 1\right\}}}{\nu^{\frac{1}{4}}\left|\eta_{1}\right|^{\frac{1}{2}}}\left\|\hat{f}_{(\mu, 0,0)}\right\|_{L_{X_{2}}^{2}}\right)
\end{array}\right\} Y_{2} \partial_{Y_{2}} \hat{h}_{\left(\mu, \frac{\rho}{s}, 0\right)}\left(\xi_{1}-\cdot, Y_{2}\right)\right\|_{L_{\eta_{1}}^{1}} .
\end{aligned}
$$


Thus, the appropriate use of the estimate like (3.4) (with $l=1$ to treat $\left|\xi_{1}\right|$ in (3.48)) and (3.12) (for the other term in (3.48)) implies that the norm $\left\|\varphi_{B}^{\left(\mu^{\prime}, \rho^{\prime} / t\right)} I_{3,2}\right\|_{L_{\xi_{1}}^{2} L_{X_{2}}^{1}}$ is bounded from above by $(\nu(t-s))^{-1 / 2}\left\{\begin{array}{l}\|f\|_{X_{I B, 1}^{(\mu, 0)}} \\ \left\|R_{1 / \nu} f\right\|_{X_{I I, 1}^{(\mu, 0)}}\end{array}\right\}\|h\|_{X_{I B, 1}^{(\mu, \rho) s}}$. The norm $\left\|\varphi_{B}^{\left(\mu^{\prime}, \rho^{\prime} / t\right)} X_{2}^{1 / 2} I_{3}\right\|_{L_{\xi_{1}}^{2} L_{X_{2}}^{2}}$ is estimated in the same way. The details are omitted here. The proof is complete.

Lemma 3.5 Assume that $0<2^{-1}\left(\mu-\mu^{\prime}\right)<\mu^{\prime}<\mu<1$ and $0<\rho^{\prime}<\rho \leq 2^{-4}$. Let $l(1)=0$ and $l(0) \in\{0,1\}$. (i) Let $j=0,1,2$. Then

$$
\left\|\Phi_{B, 2}^{(\nu)}[f, h](t-s)\right\|_{X_{I B, j}^{\left(\mu^{\prime}, \rho^{\prime}\right.}} \lesssim \frac{1}{\mu-\mu^{\prime}}\left\{\begin{array}{l}
\|f\|_{X_{I B, 2-m(j)-n(j)}^{(\mu, 0)}} \\
\left\|R_{\frac{1}{\nu}} f\right\|_{X_{I I, 2-m(j)-n(j)}^{(\mu, 0)}}
\end{array}\right\}\left\{\begin{array}{c}
\|h\|_{X_{I B, j+(1-j) n(j)}^{(\mu, 0)}} \\
\left\|R_{\frac{1}{\nu}} h\right\|_{X_{I I, j+(1-j) n(j)}^{(\mu, 0)}}
\end{array}\right\} .
$$

Here $m(j)=0$ if $j=1,2$ and $m(0)=1$, and $n(2)=0, n(1)=1$, and $n(0) \in\{0,1\}$.

(ii) Let $j=0,1$. Then

$$
\left\|\Phi_{B, 2}^{(\nu)}[f, h](t-s)\right\|_{X_{I B, j}^{\left(\mu^{\prime}, \frac{\rho^{\prime}}{t}\right)}} \lesssim \frac{1}{\nu^{\frac{1}{2}}(t-s)^{\frac{1}{2}}}\left\{\begin{array}{l}
\|f\|_{X_{I I, 1-l(j)}^{(\mu, 0)}} \\
\left\|R_{\frac{1}{\nu}} f\right\|_{X_{I I, 1-l(j)}^{(\mu, 0)}}^{(\mu)}
\end{array}\right\}\left\{\begin{array}{c}
\|h\|_{X_{I B, j+l(j)}^{(\mu, 0)}} \\
\left\|R_{\frac{1}{\nu}} h\right\|_{X_{I I, j+l(j)}^{(\mu, 0)}}^{(\mu, j)}
\end{array}\right\} .
$$

Proof. Both (3.49) and (3.50) easily follow from (3.14), (3.24), and (3.25). The proof is complete.

Lemma 3.6 Let $\phi_{r} \in C_{0}^{\infty}\left(\overline{\mathbb{R}_{+}}\right)$be a cut-off function such that $\phi_{r}\left(x_{2}\right)=1$ if $0 \leq x_{2} \leq r$ and $\phi_{r}\left(x_{2}\right)=0$ if $x_{2} \geq 2 r$. Assume that $0<2^{-1}\left(\mu-\mu^{\prime}\right)<\mu^{\prime}<\mu<1$ and $0<\rho^{\prime}<\rho \leq 2^{-4}$. Let $l(1)=0$ and $l(0) \in\{0,1\}$.

(i) Let $i=1,2$, and $j=0,1,2$. Then

$$
\left\|\phi_{t^{\frac{1}{2}}} \Psi_{i}^{(\nu)}[f, h](t-s)\right\|_{X_{I B, j}^{\left(\mu^{\prime}, \frac{\rho^{\prime}}{t}\right)}} \lesssim \frac{1}{\mu-\mu^{\prime}}\left\{\begin{array}{l}
\|f\|_{X_{I B, 2-m(j)-n(j)}^{(\mu, 0)}} \\
\left\|R_{\frac{1}{\nu}} f\right\|_{X_{I I, 2-m(j)-n(j)}^{(\mu, 0)}}
\end{array}\right\}\left\{\begin{array}{l}
\|h\|_{X_{I B, j+(1-j) n(j)}^{(\mu, 0)}} \\
\left\|R_{\frac{1}{\nu}} h\right\|_{X_{I I, j+(1-j) n(j)}^{(\mu, 0)}}
\end{array}\right\} .
$$

Here $m(j)=0$ if $j=1,2$ and $m(0)=1$, and $n(2)=0, n(1)=1$, and $n(0) \in\{0,1\}$.

(ii) Let $i=1,2$, and $j=0,1$. Then

$$
\left\|\phi_{t^{\frac{1}{2}}} \Psi_{i}^{(\nu)}[f, h](t-s)\right\|_{X_{I B, j}^{\left(\mu^{\prime}, \frac{\rho^{\prime}}{t}\right)}} \lesssim \frac{1}{\nu^{\frac{1}{2}}(t-s)^{\frac{1}{2}}}\left\{\begin{array}{l}
\|f\|_{X_{I B, 1-l(j)}^{(\mu, 0)}} \\
\left\|R_{\frac{1}{\nu}} f\right\|_{X_{I I, 1-l(j)}^{(\mu, 0)}}
\end{array}\right\}\left\{\begin{array}{c}
\|h\|_{X_{I B, j+l(j)}^{(\mu, 0)}} \\
\left\|R_{\frac{1}{\nu}} h\right\|_{X_{I, j}^{(\mu, 0)}}
\end{array}\right\} .
$$

Proof. We give the proof only for $\Psi_{1}^{(\nu)}[f, h]$, since $\Psi_{2}^{(\nu)}[f, h]$ is estimated in the same way. Using $E * f=$ $\int_{0}^{\infty} G(\tau) * f \mathrm{~d} \tau$, we have from $B^{(\nu)}(f, h)=\nabla_{X} \cdot D^{(\nu)}(f, h)$ and the integration by parts,

$$
\begin{aligned}
& \mathcal{F}\left(\Psi_{1}^{(\nu)}[f, h](t-s)\right)\left(\xi_{1}, X_{2}\right) \\
= & -2 \int_{0}^{\infty} \int_{0}^{\infty}\left(-\xi_{1}^{2}+\frac{\left|\xi_{1}\right|}{\nu^{\frac{1}{2}}} \partial_{X_{2}}\right) e^{-\{\nu(t-s)+\tau\} \xi_{1}^{2}} g\left(t-s+\frac{\tau}{\nu}, X_{2}+Y_{2}\right) \mathrm{d} \tau i \xi_{1} \mathcal{F}\left(D_{1}^{(\nu)}(f, h)\right)\left(\xi_{1}, Y_{2}\right) \mathrm{d} Y_{2} \\
& -2 \int_{0}^{\infty} e^{-\nu(t-s) \xi_{1}^{2}} g\left(t-s, X_{2}+Y_{2}\right) \nu^{\frac{1}{2}}\left|\xi_{1}\right| \mathcal{F}\left(D_{2}^{(\nu)}(f, h)\right)\left(\xi_{1}, Y_{2}\right) \mathrm{d} Y_{2} \\
& +2 \int_{0}^{\infty} \int_{0}^{\infty}\left(-\xi_{1}^{2}+\frac{\left|\xi_{1}\right|}{\nu^{\frac{1}{2}}} \partial_{X_{2}}\right) e^{-\{\nu(t-s)+\tau\} \xi_{1}^{2}} g\left(t-s+\frac{\tau}{\nu}, X_{2}+Y_{2}\right) \mathrm{d} \tau \nu^{\frac{1}{2}}\left|\xi_{1}\right| \mathcal{F}\left(D_{2}^{(\nu)}(f, h)\right)\left(\xi_{1}, Y_{2}\right) \mathrm{d} Y_{2} .
\end{aligned}
$$


Thus, by the inequality $\nu^{1 / 2}\left|\xi_{1}\right| Y_{2} \leq \nu r \xi_{1}^{2}+\frac{\left(X_{2}+Y_{2}\right)^{2}}{4 r}$ for $r, X_{2}, Y_{2} \geq 0$, we have

$$
\begin{aligned}
\left|\mathcal{F}\left(\Psi_{1}^{(\nu)}[f, h](t-s)\right)\left(\xi_{1}, X_{2}\right)\right| \lesssim & \int_{0}^{\infty}\left(\left|\xi_{1}\right|^{2}+\frac{\left|\xi_{1}\right|}{\nu^{\frac{1}{2}}\left(t-s+\frac{\tau}{\nu}\right)^{\frac{1}{2}}}\right) e^{-\frac{\nu(t-s)+\tau}{2} \xi_{1}^{2}} g\left(2\left(t-s+\frac{\tau}{\nu}\right), X_{2}\right) \mathrm{d} \tau \\
\cdot & \left(\left\|\xi_{1} e^{-\frac{1}{2} \nu^{\frac{1}{2}}\left|\xi_{1}\right| Y_{2}} \mathcal{F}\left(D_{1}^{(\nu)}(f, h)\right)\right\|_{L_{Y_{2}}^{1}}+\left\|\xi_{1} e^{-\frac{1}{2} \nu^{\frac{1}{2}}\left|\xi_{1}\right| Y_{2}} \nu^{\frac{1}{2}} \mathcal{F}\left(D_{2}^{(\nu)}(f, h)\right)\right\|_{L_{Y_{2}}^{1}}\right) \\
& +e^{-\frac{\nu(t-s)}{2} \xi_{1}^{2}} g\left(2(t-s), X_{2}\right)\left\|\xi_{1} e^{-\frac{1}{2} \nu^{\frac{1}{2}}\left|\xi_{1}\right| Y_{2}} \nu^{\frac{1}{2}} \mathcal{F}\left(D_{2}^{(\nu)}(f, h)\right)\right\|_{L_{Y_{2}}^{1}} . \quad(3.53)
\end{aligned}
$$

The terms $\left\|\xi_{1} e^{-\frac{1}{2} \nu^{1 / 2}\left|\xi_{1}\right| Y_{2}} \mathcal{F}\left(D_{1}^{(\nu)}(f, h)\right)\right\|_{L_{Y_{2}}^{1}}$ and $\left\|\xi_{1} e^{-\frac{1}{2} \nu^{1 / 2}\left|\xi_{1}\right| Y_{2}} \nu^{1 / 2} \mathcal{F}\left(D_{2}^{(\nu)}(f, h)\right)\right\|_{L_{Y_{2}}^{1}}$ are estimated in the same way as $\mathcal{F}\left(N^{(\nu)}(f, h)\right)$ in the proof of Lemma 3.3 (see (3.34)), and hence, it follows that

$$
\begin{aligned}
& \left\|e^{\frac{\mu^{\prime}}{4}\left|\xi_{1}\right|} \xi_{1}^{1+j} e^{-\frac{1}{2} \nu \frac{1}{2}\left|\xi_{1}\right| Y_{2}} \mathcal{F}\left(D_{1}^{(\nu)}(f, h)\right)\right\|_{L_{\xi_{1}}^{2} L_{Y_{2}}^{1}}+\left\|e^{\frac{\mu^{\prime}}{4}\left|\xi_{1}\right|} \xi_{1}^{1+j} e^{-\frac{1}{2} \nu^{\frac{1}{2}}\left|\xi_{1}\right| Y_{2}} \nu^{\frac{1}{2}} \mathcal{F}\left(D_{2}^{(\nu)}(f, h)\right)\right\|_{L_{\xi_{1}}^{2} L_{Y_{2}}^{1}} \\
\lesssim & \frac{1}{\mu-\mu^{\prime}}\left\{\begin{array}{c}
\|f\|_{X_{I B, 2-m(j)-n(j)}^{(\mu, 0)}} \\
\left\|R_{\frac{1}{\nu}} f\right\|_{X_{I I, 2-m(j)-n(j)}^{(\mu, 0)}}
\end{array}\right\}\left\{\begin{array}{c}
\|h\|_{X_{I B, j+(1-j) n(j)}^{(\mu, 0)}}\left\|R_{\frac{1}{\nu}} h\right\|_{X_{I I, j+(1-j) n(j)}^{(\mu, 0)}}^{(\mu)}
\end{array}\right\}
\end{aligned}
$$

for $j=0,1,2$. Thus we see from the inequality $\int_{0}^{\infty}\left(\xi_{1}^{2}+\frac{\left|\xi_{1}\right|}{\nu^{1 / 2}(t-s+\tau / \nu)^{1 / 2}}\right) e^{-\frac{\nu(t-s)+\tau}{2} \xi_{1}^{2}} \mathrm{~d} \tau \leq C$ that $\left\|\varphi_{B}^{\left(\mu^{\prime}, \rho^{\prime} / t\right)} \xi_{1}^{j} X_{2}^{k / 2} \phi_{t^{1 / 2}} \mathcal{F}\left(\Psi_{1}^{(\nu)}[f, h](t-s)\right)\right\|_{L_{\xi_{1}}^{2} L_{X_{2}}^{1+k}}$ is bounded from above by the right-hand side of (3.51). The estimate for $X_{2} \partial_{X_{2}}\left\{\phi_{t^{1 / 2}} \mathcal{F}\left(\Psi_{1}^{(\nu)}[f, h](t-s)\right)\right\}$ is proved in the same manner by using the inequality $\left|X_{2}^{l} \partial_{X_{2}}^{l} g\left(t-s+\tau / \nu, X_{2}+Y_{2}\right)\right| \lesssim g\left(5(t-s+\tau / \nu) / 4, X_{2}\right), l=1,2$. Now (3.51) has been proved. The estimate (3.52) for $\Psi_{1}^{(\nu)}[f, h]$ is proved similarly. Indeed, in this case the terms $\left\|\xi_{1} e^{-\frac{1}{2} \nu^{1 / 2}\left|\xi_{1}\right| Y_{2}} \mathcal{F}\left(D_{1}^{(\nu)}(f, h)\right)\right\|_{L_{Y_{2}}^{1}}$ and $\left\|\xi_{1} e^{-\frac{1}{2} \nu^{1 / 2}\left|\xi_{1}\right| Y_{2}} \nu^{1 / 2} \mathcal{F}\left(D_{2}^{(\nu)}(f, h)\right)\right\|_{L_{Y_{2}}^{1}}$ in (3.53) are estimated in the same way as (3.35), and we also use the inequality $\left|\xi_{1}\right| e^{-\frac{\nu(t-s)+\tau}{4} \xi_{1}^{2}} \lesssim(\nu(t-s))^{-1 / 2}$. The details are omitted here. The proof is complete.

Lemma 3.7 Let $\phi_{r}^{c}=1-\phi_{r}$, where $\phi_{r}$ is the function in Lemma 3.6. Assume that $0<2^{-1}\left(\mu-\mu^{\prime}\right)<$ $\mu^{\prime}<\mu<1$ and $0<\rho^{\prime}<\rho \leq 2^{-4}$. Let $l(1)=0$ and $l(0) \in\{0,1\}$.

(i) Let $i=1,2$, and $j=0,1,2$. Then

$\left\|\phi_{t^{\frac{1}{2}}}^{c} \Upsilon_{i}^{(\nu)}[f, h](s)\right\|_{X_{I B, j}^{\left(\mu^{\prime}, \rho^{\prime}\right)}} \lesssim \frac{1}{\mu-\mu^{\prime}}\left\{\begin{array}{l}\|f\|_{L^{\infty}\left(0, s ; X_{I B, 2-m(j)-n(j)}^{(\mu, 0)}\right)} \\ \left\|R_{\frac{1}{\nu}} f\right\|_{L^{\infty}\left(0, s ; X_{I I, 2-m(j)-n(j)}^{(\mu, 0)}\right)}\end{array}\right\}\left\{\begin{array}{l}\|h\|_{L^{\infty}\left(0, s ; X_{I B, j+(1-j) n(j)}^{(\mu, 0)}\right)} \\ \left\|R_{\frac{1}{\nu}} h\right\|_{L^{\infty}\left(0, s ; X_{I I, j+(1-j) n(j)}^{(\mu, 0)}\right)}\end{array}\right\}$.

Here $m(j)=0$ if $j=1,2$ and $m(0)=1$, and $n(2)=0, n(1)=1$, and $n(0) \in\{0,1\}$.

(ii) Let $i=1,2$, and $j=0,1$. Then

$$
\left\|\phi_{t^{\frac{1}{2}}}^{c} \Upsilon_{i}^{(\nu)}[f, h](s)\right\|_{X_{I B, j}^{\left(\mu^{\prime}, \rho_{t}^{\prime}\right)}} \lesssim \frac{1}{\nu^{\frac{1}{2}}(t-s)^{\frac{1}{2}}}\left\{\begin{array}{l}
\|f\|_{L^{\infty}\left(0, s ; X_{I B, 1-l(j)}^{(\mu, 0)}\right)} \\
\left\|R_{\frac{1}{\nu}} f\right\|_{L^{\infty}\left(0, s ; X_{I I, 1-l(j)}^{(\mu, 0)}\right)}
\end{array}\right\}\left\{\begin{array}{l}
\|h\|_{L^{\infty}\left(0, s ; X_{I B, j+l(j)}^{(\mu, 0)}\right)} \\
\left\|R_{\frac{1}{\nu}} h\right\|_{L^{\infty}\left(0, s ; X_{I I, j+l(j)}^{(\mu, 0)}\right)}
\end{array}\right\} .
$$


Proof. We give the proof only for $\Upsilon_{1}^{(\nu)}[f, h]$ since $\Upsilon_{2}^{(\nu)}[f, h]$ is estimated similarly. By the definition of $\Upsilon_{1}^{(\nu)}[f, h]$ and $B^{(\nu)}(f, h)=\nabla_{X} \cdot D^{(\nu)}(f, h)$ the integration by parts yields

$$
\begin{aligned}
\mathcal{F}\left(\Upsilon_{1}^{(\nu)}[f, h]\right)\left(\xi_{1}, X_{2}\right)= & 2 \int_{0}^{s} \int_{0}^{\infty}\left(-\nu \xi_{1}^{2}+\nu^{\frac{1}{2}}\left|\xi_{1}\right| \partial_{X_{2}}\right) e^{-\nu(s-\tau) \xi_{1}^{2}} g\left(s-\tau, X_{2}+Y_{2}\right) i \xi_{1} \mathcal{F}\left(D_{1}^{(\nu)}(f, h)\right) \mathrm{d} Y_{2} \mathrm{~d} \tau \\
& -2 \int_{0}^{s} \int_{0}^{\infty}\left(-\nu \xi_{1}^{2}+\nu^{\frac{1}{2}}\left|\xi_{1}\right| \partial_{X_{2}}\right) e^{-\nu(s-\tau) \xi_{1}^{2}} \partial_{Y_{2}} g\left(s-\tau, X_{2}+Y_{2}\right) \mathcal{F}\left(D_{2}^{(\nu)}(f, h)\right) \mathrm{d} Y_{2} \mathrm{~d} \tau .
\end{aligned}
$$

We use the inequality $\left(\nu^{1 / 2}\left|\xi_{1}\right|\right)^{l}\left|\partial_{X_{2}}^{2-l} e^{-\nu(s-\tau) \xi_{1}^{2}} g\left(s-\tau, X_{2}+Y_{2}\right)\right| \lesssim t^{-1} e^{-\frac{\nu(s-\tau)}{3} \xi_{1}^{2}-\frac{1}{2} \nu^{1 / 2}\left|\xi_{1}\right| Y_{2}} g\left(3(s-\tau), X_{2}\right)$ for $X_{2} \geq t^{1 / 2}$ and $l=0,1,2$, to get

$$
\begin{aligned}
\phi_{t^{\frac{1}{2}}}^{c}\left|\mathcal{F}\left(\Upsilon_{1}^{(\nu)}[f, h]\right)\left(\xi_{1}, X_{2}\right)\right| \lesssim \int_{0}^{s} e^{-\frac{1}{3} \nu(s-\tau) \xi_{1}^{2}} g\left(3(s-\tau), X_{2}\right) \mathrm{d} \tau \\
\cdot\left(\left\|\xi_{1} e^{-\frac{1}{2} \nu^{\frac{1}{2}}\left|\xi_{1}\right| Y_{2}} \mathcal{F}\left(D_{1}^{(\nu)}(f, h)\right)\right\|_{L_{Y_{2}}^{1}}+\left\|\xi_{1} e^{-\frac{1}{2} \nu^{\frac{1}{2}}\left|\xi_{1}\right| Y_{2}} \nu^{\frac{1}{2}} \mathcal{F}\left(D_{2}^{(\nu)}(f, h)\right)\right\|_{L_{Y_{2}}^{1}}\right) .
\end{aligned}
$$

The integrand in the right-hand side of (3.56) is estimated as in Lemma 3.6. Hence we see, for example, $\left\|\varphi_{B}^{\left(\mu^{\prime}, \frac{\rho^{\prime}}{t}\right)} \xi_{1}^{j} X_{2}^{\frac{k}{2}} \phi_{t^{\frac{1}{2}}}^{c} \mathcal{F}\left(\Upsilon_{1}^{(\nu)}[f, h]\right)\right\|_{L_{\xi_{1}}^{2} L_{X_{2}}^{1+k}} \lesssim \frac{1}{\mu-\mu^{\prime}}\left\{\begin{array}{l}\|f\|_{L^{\infty}\left(0, s ; X_{I B, \tau(j)}^{(\mu, 0)}\right)} \\ \left\|R_{\frac{1}{\nu}} f\right\|_{L^{\infty}\left(0, s ; X_{I, \tau}^{(\mu, 0)}\right)}\end{array}\right\}\left\{\begin{array}{l}\|h\|_{L^{\infty}\left(0, s ; X_{I B, \tau^{\prime}(j)}^{(\mu, 0)}\right)} \\ \left\|R_{\frac{1}{\nu}} h\right\|_{L^{\infty}\left(0, s ; X_{I I, \tau^{\prime}(j)}^{(\mu, 0)}\right)}\end{array}\right\}$.

Here we have set $\tau(j)=2-m(j)-n(j)$ and $\tau^{\prime}(j)=j+(1-j) n(j)$. The other norms are estimated in the same way. The proof is complete.

In the proofs of Lemma $3.8-3.10$ below we set $\hat{f}_{(\mu, \rho, \theta)}\left(\xi_{1}, x_{2}\right)=\varphi_{B}^{(0, \rho)}\left(\xi_{1}, x_{2} / \nu^{1 / 2}\right) \varphi_{I}^{(\mu, \theta)}\left(\xi_{1}, x_{2}\right) \hat{f}\left(\xi_{1}, x_{2}\right)$.

Lemma 3.8 Assume that $0<\mu \leq d_{E}$ and $0<\theta^{\prime}<\theta \leq 2^{-8}$. Let $j=0,1$, and let $l(1)=0$ and $l(0) \in\{0,1\}$. Then

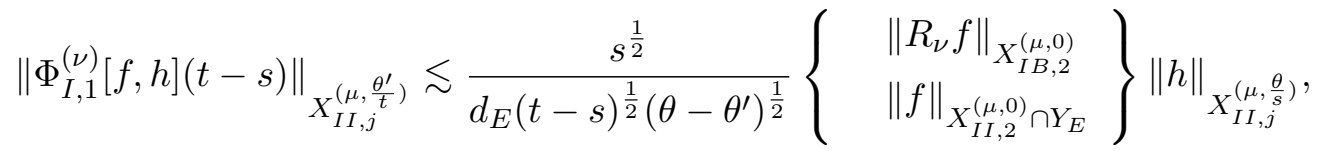

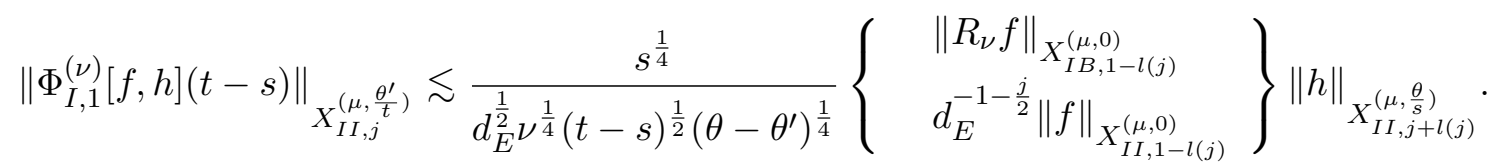

Proof. We will write $\|\hat{f}\|_{L_{x_{2}}^{p}}$ instead of $\left\|\hat{f}\left(\eta_{1}\right)\right\|_{L_{x_{2}}^{p}}$ for short. Lemma 2.4 imply for $i=1,2$,

$$
\begin{aligned}
& \left|\chi_{4 d_{E}} \mathcal{F}\left(D_{i}(f, h)\right)\left(\xi_{1}, y_{2}\right)\right| \\
\lesssim & \chi_{4 d_{E}} \int_{\mathbb{R}}\left(\int_{0}^{7 d_{E}} e^{-\left|\eta_{1}\right|\left|y_{2}-z_{2}\right|}\left|\hat{f}\left(\eta_{1}, z_{2}\right)\right| \mathrm{d} z_{2}+\int_{7 d_{E}}^{\infty} e^{-\left|\eta_{1}\right|\left|y_{2}-z_{2}\right|}\left|\hat{f}\left(\eta_{1}, z_{2}\right)\right| \mathrm{d} z_{2}\right)\left|\hat{h}\left(\xi_{1}-\eta_{1}, y_{2}\right)\right| \mathrm{d} \eta_{1} \\
\lesssim & \chi_{4 d_{E}} \int_{\mathbb{R}}\left(e^{-\frac{1}{4}\left(\mu-y_{2}\right)_{+}\left|\eta_{1}\right|}\left\|\hat{f}_{(\mu, 0,0)} \chi_{7 d_{E}}\right\|_{L_{x_{2}}^{1}}+e^{-2 d_{E}\left|\eta_{1}\right|}\left\|\hat{f} \chi_{6 d_{E}}^{c}\right\|_{L_{x_{2}}^{1}}\right)\left|\hat{h}\left(\xi_{1}-\eta_{1}, y_{2}\right)\right| \mathrm{d} \eta_{1} \\
\lesssim & e^{-\frac{1}{4}\left(\mu-y_{2}\right)+\left|\xi_{1}\right|-\frac{\theta}{\nu s}\left(6 d_{E}-y_{2}\right)_{+}^{2}} \chi_{4 d_{E}}\left\|\left(\left\|\hat{f}_{(\mu, 0,0)} \chi_{7 d_{E}}\right\|_{L_{x_{2}}^{1}}+e^{-d_{E}\left|\eta_{1}\right|}\left\|\hat{f} \chi_{6 d_{E}}^{c}\right\|_{L_{x_{2}}^{1}}\right) \mid \hat{h}_{\left(\mu, 0, \frac{\theta}{s}\right)}\left(\xi_{1}-\cdot, y_{2}\right)\right\|_{L_{\eta_{1}}^{1}} .
\end{aligned}
$$

Thus, from $\left\|\hat{f}_{(\mu, 0,0)} \chi_{7 d_{E}}\right\|_{L_{x_{2}}^{1}} \lesssim\left\|\hat{f}_{(\mu, 0,0)}\right\|_{L_{x_{2}}^{2}}$ and $\left\|\hat{f} \chi_{6 d_{E}}^{c}\right\|_{L_{x_{2}}^{1}} \leq\left\langle\eta_{1}\right\rangle^{-4}\|f\|_{Y_{E}}$ for $f \in X_{I I, 2}^{(\mu, 0)} \cap Y_{E}$, 
we have $\left\|\varphi_{I}^{(\mu, \theta / s)} \xi_{1}^{j} \chi_{4 d_{E}} \mathcal{F}\left(D_{i}(f, h)\right)\right\|_{L_{\xi_{1}}^{2} L_{y_{2}}^{2}} \lesssim\left\{\begin{array}{c}\left\|R_{\nu} f\right\|_{X_{I B, 2}^{(\mu, 0)}} \\ \|f\|_{X_{I I, 2}^{\mu, 0)} \cap Y_{E}}\end{array}\right\}\|h\|_{X_{I I, j}^{(\mu, \theta / s)}}$. Let $e^{t \Delta_{D}}$ be the heat semigroup with the unit viscosity in the half plane subject to the homogeneous Dirichlet boundary condition. From the integration by parts and $\mathcal{F}\left(D_{2}(f, h)\right)\left(\xi_{1}, 0\right)=0$ we see that $\left|\mathcal{F}\left(\Phi_{I, 1}^{(\nu)}[f, h]\right)\right|$ is bounded from above by $\left|\xi_{1} \mathcal{F}\left(e^{\nu(t-s) \Delta_{N}}\left(\chi_{4 d_{E}} D_{1}(f, h)\right)\right)\right|+\left|\partial_{2} \mathcal{F}\left(e^{\nu(t-s) \Delta_{D}}\left(\chi_{4 d_{E}} D_{2}(f, h)\right)\right)\right|$. Since (3.7) holds even if $\Delta_{N}$ is replaced by $\Delta_{D}$, by applying it with $\gamma=1$ we have for $j=0,1$,

$$
\left\|\varphi_{I}^{\left(\mu, \frac{\theta}{t}\right)} \xi_{1}^{j} \mathcal{F}\left(\Phi_{I, 1}^{(\nu)}[f, h]\right)\right\|_{L_{\xi_{1}}^{2} L_{x_{2}}^{2}} \lesssim \frac{s^{\frac{1}{2}}}{d_{E}(t-s)^{\frac{1}{2}}\left(\theta-\theta^{\prime}\right)^{\frac{1}{2}}}\left\{\begin{array}{l}
\left\|R_{\nu} f\right\|_{X_{I B, 2}^{(\mu, 0)}} \\
\|f\|_{X_{I, 2}^{(\mu, 0)} \cap Y_{E}}
\end{array}\right\}\|h\|_{X_{I I, j}^{\left(\mu, \frac{\theta}{s}\right)}}
$$

While, from the estimates $\left\|\hat{f}_{(\mu, 0,0)} \chi_{7 d_{E}}\right\|_{L_{x_{2}}^{1}} \lesssim\left\|\hat{f}_{(\mu, 0,0)}\right\|_{L_{x_{2}}^{2}},\left\|\hat{f}_{6 d_{E}}^{c}\right\|_{L_{\eta_{1}}^{\infty} L_{x_{2}}^{1}} \leq\|f\|_{X_{I I, 0}^{(0,0)}}$ for $f \in X_{I I, 0}^{(\mu, 0)}$ and $d_{E} \in(0,1 / 2)$, we see $\left\|\varphi_{I}^{(\mu, \theta / s)} \xi_{1}^{j} \chi_{4 d_{E}} \mathcal{F}\left(D_{i}(f, h)\right)\right\|_{L_{\xi_{1}}^{2} L_{x_{2}}^{2}} \lesssim\left\{\begin{array}{c}\left\|R_{\nu} f\right\|_{X_{I B, 1-l(j)}^{(\mu, 0)}} \\ d_{E}^{-1-j / 2}\|f\|_{X_{I I, 1-l(j)}^{\mu, 0)}}\end{array}\right\}\|h\|_{X_{I I, j+l(j)}^{(\mu, \theta / s)}}$ for $i=1,2$. Hence from (3.7) with $\gamma=1 / 2$ we arrive at

$$
\left\|\varphi_{I}^{\left(\mu, \theta^{\prime}\right)} \xi_{1}^{j} \mathcal{F}\left(\Phi_{I, 1}^{(\nu)}[f, h]\right)\right\|_{L^{2}} \lesssim \frac{s^{\frac{1}{4}}}{\nu^{\frac{1}{4}} d_{E}^{\frac{1}{2}}(t-s)^{\frac{1}{2}}\left(\theta-\theta^{\prime}\right)^{\frac{1}{4}}}\left\{\begin{array}{c}
\left\|R_{\frac{1}{\nu}} f\right\|_{X_{I B, 1-l(j)}^{(\mu, 0)}} \\
d_{E}^{-1-\frac{j}{2}}\|f\|_{X_{I I, 1-l(j)}^{(\mu, 0)}}
\end{array}\right\}\|h\|_{X_{I I, j \rightarrow l(j)}^{\left(\mu, \frac{\theta}{s}\right)}},
$$

for $j=0,1$. To estimate $\partial_{2} \mathcal{F}\left(\Phi_{I, 1}^{(\nu)}[f, h]\right)$ we observe from the similar calculations as above that

$$
\begin{aligned}
& \varphi_{I}^{\left(\mu, \frac{\theta}{s}\right)}\left|\mathcal{F}\left(B_{1}\left(f, \chi_{4 d_{E}} h\right)\right)\left(\xi_{1}, y_{2}\right)\right| \\
\lesssim & \chi_{4 d_{E}} \int_{\mathbb{R}}\left(\left\|\hat{f}_{(\mu, 0,0)} \chi_{7 d_{E}}\right\|_{L_{x_{2}}^{1}}+e^{-d_{E}\left|\eta_{1}\right|}\left\|\hat{f} \chi_{6 d_{E}}^{c}\right\|_{L_{x_{2}}^{1}}\right)\left|\left(\xi_{1}-\eta_{1}\right) \hat{h}_{\left(\mu, 0, \frac{\theta}{s}\right)}\left(\xi_{1}-\eta_{1}, y_{2}\right)\right| \mathrm{d} \eta_{1},
\end{aligned}
$$

and

$$
\begin{aligned}
\varphi_{I}^{\left(\mu, \frac{\theta}{s}\right)}\left|\mathcal{F}\left(B_{2}\left(f, \chi_{4 d_{E}} h\right)\right)\left(\xi_{1}, y_{2}\right)\right| \lesssim \int_{\mathbb{R}}\left(\left\|\hat{f}_{(\mu, 0,0)} \chi_{7 d_{E}}\right\|_{L_{x_{2}}^{1}}+e^{-d_{E}\left|\eta_{1}\right|}\left\|\hat{f} \chi_{6 d_{E}}^{c}\right\|_{L_{x_{2}}^{1}}\right) \\
\cdot\left(\left|\eta_{1} y_{2} \partial_{2} \chi_{4 d_{E}} \hat{h}_{\left(\mu, 0, \frac{\theta}{s}\right)}\left(\xi_{1}-\eta_{1}, y_{2}\right)\right|+\chi_{4 d_{E}}\left|\partial_{2} \hat{h}_{\left(\mu, 0, \frac{\theta}{s}\right)}\left(\xi_{1}-\eta_{1}, y_{2}\right)\right|\right) \mathrm{d} \eta_{1} .
\end{aligned}
$$

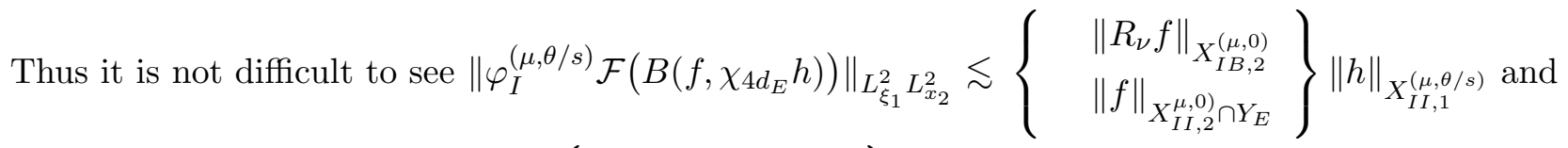
$\left\|\varphi_{I}^{(\mu, \theta / s)} \mathcal{F}\left(B\left(f, \chi_{4 d_{E}} h\right)\right)\right\|_{L_{\xi_{1}}^{2} L_{x_{2}}^{2}} \lesssim\left\{\begin{array}{c}\left\|R_{\nu} f\right\|_{X_{I B, 1}^{(\mu, 0)}} \\ d_{E}^{-3 / 2}\|f\|_{X_{I I, 1}^{\mu, 0)}}\end{array}\right\}\|h\|_{X_{I I, 1}^{(\mu, \theta / s)}}$. So as in the proof of (3.59) and (3.60), we get the desired estimates for $\left\|\varphi_{I}^{\left(\mu, \theta^{\prime} / t\right)} \partial_{2} \mathcal{F}\left(\Phi_{I, 1}^{(\nu)}[f, h]\right)\right\|_{L^{2}}$. Finally let us estimate the $L^{1}$ norm of $\varphi_{I}^{\left(0, \theta^{\prime} / t\right)} \Phi_{I, 1}^{(\nu)}[f, h]$. From the definition of $D(f, h)$ and the integration by parts we have

$$
\begin{aligned}
\left|\Phi_{I, 1}^{(\nu)}[f, h](t-s)(x)\right| & \lesssim \frac{1}{\nu^{\frac{1}{2}}(t-s)^{\frac{1}{2}}} \int_{\mathbb{R}_{+}^{2}} G(2 \nu(t-s), x-y)\left|D\left(f, \chi_{4 d_{E}} h\right)(y)\right| \mathrm{d} y \\
& \lesssim \frac{s^{\frac{1}{2}}}{d_{E}(t-s)^{\frac{1}{2}}\left(\theta-\theta^{\prime}\right)^{\frac{1}{2}}} \int_{\mathbb{R}_{+}^{2}} G(2 \nu(t-s), x-y) \varphi_{I}^{\left(0, \frac{\theta-\theta^{\prime}}{s}\right)}\left|\chi_{4 d_{E}} h J(f)(y)\right| \mathrm{d} y .
\end{aligned}
$$


Thus we have from (3.13) and by applying Lemma 2.5 after $\left\|J(f) h_{(0,0, \theta / s)}\right\|_{L^{1}} \leq\|J(f)\|_{L^{4}}\left\|h_{(0,0, \theta / s)}\right\|_{L^{4 / 3}}$,

$$
\left\|\varphi_{I}^{\left(0, \frac{\theta^{\prime}}{t}\right)} \Phi_{I, 1}^{(\nu)}[f, h]\right\|_{L^{1}} \lesssim \frac{s^{\frac{1}{2}}\left\|J(f) h_{\left(0,0, \frac{\theta}{s}\right)}\right\|_{L^{1}}}{d_{E}(t-s)^{\frac{1}{2}}\left(\theta-\theta^{\prime}\right)^{\frac{1}{2}}} \lesssim \frac{s^{\frac{1}{2}}}{d_{E}(t-s)^{\frac{1}{2}}\left(\theta-\theta^{\prime}\right)^{\frac{1}{2}}}\left\{\begin{array}{c}
\left\|R_{\nu} f\right\|_{X_{I B, 0}^{(0,0)}} \\
\|f\|_{X_{I I, 0}^{(0,0)}}
\end{array}\right\}\|h\|_{X_{I I, 0}^{\left(0, \frac{\theta}{s}\right)}} .
$$

Similarly, one can derive

$$
\left\|\varphi_{I}^{\left(0, \frac{\theta^{\prime}}{t}\right)} \Phi_{I, 1}^{(\nu)}[f, h]\right\|_{L^{1}} \lesssim \frac{s^{\frac{1}{4}}}{\nu^{\frac{1}{4}} d_{E}^{\frac{1}{2}}(t-s)^{\frac{1}{2}}\left(\theta-\theta^{\prime}\right)^{\frac{1}{4}}}\left\{\begin{array}{c}
\left\|R_{\nu} f\right\|_{X_{I B, 0}^{(0,0)}} \\
\|f\|_{X_{I I, 0}^{(0,0)}}
\end{array}\right\}\|h\|_{X_{I I, 0}^{\left(0, \frac{\theta}{s}\right)}} .
$$

The details are omitted here. This completes the proof.

Lemma 3.9 Assume that $0<\mu \leq d_{E}$ and $0<\theta \leq 2^{-8}$. Let $j=0,1$, and let $l(1)=0$ and $l(0) \in\{0,1\}$. Then

$$
\left\|\Phi_{I, 2}^{(\nu)}[f, h](t-s)\right\|_{X_{I I, j}^{\left(\mu, \frac{\theta}{t}\right)}} \lesssim \frac{1}{\nu^{\frac{1}{2}}(t-s)^{\frac{1}{2}}} e^{\frac{1}{64 \nu s} d_{E}^{2}}\left\{\begin{array}{l}
\left\|R_{\nu} f\right\|_{X_{I B, 1-l(j)}^{(0,0)}} \\
d_{E}^{-1-\frac{j}{2}}\|f\|_{X_{I I, 1-l(j)}^{(0,0)}}
\end{array}\right\}\left\|\chi_{4 d_{E}}^{c} \chi_{8 d_{E}} h\right\|_{W^{j+l(j), 2} \cap L^{\frac{4}{3}}} .
$$

Proof. From

$$
e^{-\frac{1}{2} \nu(t-s) \xi_{1}^{2}-\frac{\left(x_{2}-y_{2}\right)^{2}}{8 \nu(t-s)}} \leq e^{-\frac{1}{2} \nu^{\frac{1}{2}}\left|x_{2}-y_{2}\right|\left|\xi_{1}\right|} \leq e^{-\frac{1}{2} \nu^{\frac{1}{2}}\left(d_{E}-x_{2}\right)+\left|\xi_{1}\right|} \quad y_{2} \geq d_{E},
$$

by arguing as in the proof of Lemma 3.8 we have with $\chi=\chi_{4 d_{E}}^{c} \chi_{8 d_{E}}$,

$$
\begin{aligned}
\left|\mathcal{F}\left(\Phi_{I, 2}^{(\nu)}[f, h](t-s)\right)\left(\xi_{1}, x_{2}\right)\right| \lesssim & \frac{1}{\nu^{\frac{1}{2}}(t-s)^{\frac{1}{2}}} e^{-\frac{1}{4} \nu(t-s) \xi_{1}^{2}-\frac{1}{2}\left(d_{E}-x_{2}\right)_{+}\left|\xi_{1}\right|} \int_{0}^{\infty} g\left(4 \nu(t-s), x_{2}-y_{2}\right) \\
& \cdot \int_{\mathbb{R}}\left(\left\|\hat{f} \chi_{11 d_{E}}\right\|_{L_{x_{2}}^{1}}+e^{-d_{E}\left|\eta_{1}\right|}\left\|\hat{f} \chi_{10 d_{E}}^{c}\right\|_{L_{x_{2}}^{1}}\right)\left|\hat{h}\left(\xi_{1}-\eta_{1}, y_{2}\right)\right| \chi \mathrm{d} \eta_{1} \mathrm{~d} y_{2} .
\end{aligned}
$$

Thus, as in the proof of (3.6), one can derive

$$
\begin{aligned}
&\left\|\varphi_{I}^{\left(\mu^{\prime}, \theta^{\prime}\right)} \xi_{1}^{j} \mathcal{F}\left(\Phi_{I, 2}^{(\nu)}[f, h]\right)\right\|_{L_{\xi_{1}}^{2} L_{x_{2}}^{2}} \lesssim \frac{1}{\nu^{\frac{1}{2}}(t-s)^{\frac{1}{2}}}\left\{\begin{array}{c}
\left\|R_{\nu} f\right\|_{X_{I B, 1-l(j)}^{(0,0)}} \\
d_{E}^{-1-\frac{j}{2}}\|f\|_{X_{I I, 1-l(j)}^{(0,0)}}
\end{array}\right\}\left\|\left\langle\xi_{1}\right\rangle^{j+l(j)} \hat{h}_{(0,0, \theta)} \chi\right\|_{L_{\xi_{1}}^{2} L_{x_{2}}^{2}} \\
& \lesssim \frac{1}{\nu^{\frac{1}{2}}(t-s)^{\frac{1}{2}}} e^{\frac{4 \theta}{\nu s} d_{E}^{2}}\left\{\begin{array}{c}
\left\|R_{\nu} f\right\|_{X_{I B, 1-l(j)}^{(0,0)}} \\
d_{E}^{-1-\frac{j}{2}}\|f\|_{X_{I I, 1-l(j)}^{(0,0)}}
\end{array}\right\}\|\chi h\|_{W^{j+l(j), 2}}
\end{aligned}
$$

Here we have used $\left\|\hat{f} \chi_{10 d_{E}}^{c}\right\|_{L_{\eta_{1}}^{\infty} L_{x_{2}}^{1}} \leq\|f\|_{L^{1}}$ for $f \in X_{I I, 0}^{(0,0)}$, and also used the Parseval equality and $\varphi_{I}^{(0, \theta / s)} \leq e^{\frac{4 \theta}{\nu s} d_{E}^{2}}$ for $y_{2} \geq 4 d_{E}$ in the last line. The similar argument using the estimate like (3.63) yields

$$
\left\|\varphi_{I}^{(\mu, \theta)} \Phi_{I, 2}^{(\nu)}[f, h]\right\|_{L^{1}} \lesssim \frac{1}{\nu^{\frac{1}{2}}(t-s)^{\frac{1}{2}}} e^{\frac{4 \theta}{\nu s} d_{E}^{2}}\left\{\begin{array}{l}
\left\|R_{\nu} f\right\|_{X_{I B, 0}^{(0,0)}} \\
\|f\|_{X_{I I, 0}^{(0,0)}}
\end{array}\right\}\left\|\chi_{4 d_{E}}^{c} \chi_{8 d_{E}} h\right\|_{L^{\frac{4}{3}}} .
$$

In particular, (3.64) with $j=0$ follows from $\theta \leq 2^{-8}$. From the definition of $B(f, h)$ and (3.65) the term $\partial_{2} \mathcal{F}\left(\Phi_{I, 2}^{(\nu)}[f, h]\right)$ is estimated in the same way (see also the arguments in (3.61) - (3.62)). The details are omitted. The proof is complete. 
Lemma 3.10 Assume that $0<\mu \leq d_{E}, \rho>0$, and $0<\theta \leq 2^{-8}$. Let $j=0,1$, and let $l(1)=0$ and $l(0) \in\{0,1\}$. Then

$$
\begin{aligned}
& \left\|\Phi_{I, 3}^{(\nu)}[f, h](t-s)\right\|_{X_{I, j}^{\left(\mu, \frac{\theta}{t}\right)}} \\
\lesssim & \left\{\begin{array}{l}
\left(d_{E}^{-1-\frac{j}{2}}\left(\frac{\nu s}{\rho}\right)^{\frac{1}{2}}+j d_{E}^{-1} e^{-\frac{\rho}{\nu s} d_{E}^{2}}\right)\left\|R_{\nu} f\right\|_{X_{I B, 1-l(j)}^{\left(0, \frac{\rho}{s}\right)}} \\
\|f\|_{X_{I I, 1-l(j)}^{(0,0)}}
\end{array}\right\}\left(\left\|\nabla\left(\chi_{8 d_{E}}^{c} h\right)\right\|_{W^{j+l(j), 2}}+\left\|\nabla\left(\chi_{8 d_{E}}^{c} h\right)\right\|_{L^{\frac{4}{3}}}\right) .
\end{aligned}
$$

Proof. First we note that $\varphi_{I}^{(\mu, \theta / t)} \mathcal{F}\left(B\left(f, \chi_{8 d_{E}}^{c} h\right)\right)=\mathcal{F}\left(B\left(f, \chi_{8 d_{E}}^{c} h\right)\right)$. Combining this with (3.6), we get

$$
\left\|\varphi_{I}^{\left(\mu, \frac{\theta}{t}\right)} \xi_{1}^{j} \mathcal{F}\left(\Phi_{I, 3}^{(\nu)}[f, h](t-s)\right)\right\|_{L_{\xi_{1}}^{2} L_{x_{2}}^{2}} \lesssim\left\|\xi_{1}^{j} \mathcal{F}\left(B\left(f, \chi_{8 d_{E}}^{c} h\right)\right)\right\|_{L_{\xi_{1}}^{2} L_{x_{2}}^{2}}=\left\|\partial_{1}^{j} B\left(f, \chi_{8 d_{E}}^{c} h\right)\right\|_{L_{x}^{2}} .
$$

When $j=0$ by using Lemma 2.5 the last term is estimated in two ways:

$$
\begin{aligned}
& \left\|B\left(f, \chi_{8 d_{E}}^{c} h\right)\right\|_{L_{x}^{2}} \lesssim\left\|\chi_{4 d_{E}}^{c} J(f)\right\|_{L^{\infty}}\left\|\nabla\left(\chi_{8 d_{E}}^{c} h\right)\right\|_{L^{2}} \lesssim\left\{\begin{array}{l}
d_{E}^{-\frac{3}{2}}\left(\frac{\nu s}{\rho}\right)^{\frac{1}{2}}\left\|R_{\nu} f\right\|_{X_{I B, 1}^{\left(0, \frac{\rho}{s}\right)}} \\
\|f\|_{X_{I I, 1}^{(0,0)}}^{(0,0)}
\end{array}\right\}\left\|\nabla\left(\chi_{8 d_{E}}^{c} h\right)\right\|_{L^{2}}, \\
& \left\|B\left(f, \chi_{8 d_{E}}^{c} h\right)\right\|_{L_{x}^{2}} \lesssim\left\|\chi_{4 d_{E}}^{c} J(f)\right\|_{L^{4}}\left\|\nabla\left(\chi_{8 d_{E}}^{c} h\right)\right\|_{L^{4}} \lesssim\left\{\begin{array}{l}
d_{E}^{-1}\left(\frac{\nu s}{\rho}\right)^{\frac{1}{2}}\left\|R_{\nu} f\right\|_{X_{I B, 0}^{\left(0, \frac{\rho}{s}\right)}} \\
\|f\|_{X_{I I, 0}^{(0,0)}}
\end{array}\right\}\left\|\nabla\left(\chi_{8 d_{E}}^{c} h\right)\right\|_{W^{1,2},} .
\end{aligned}
$$

When $j=1$ it follows again from Lemma 2.5 that

$$
\begin{aligned}
\left\|\partial_{1} B\left(f, \chi_{8 d_{E}}^{c} h\right)\right\|_{L_{x}^{2}} & \lesssim\left\|\chi_{4 d_{E}}^{c} J\left(\partial_{1} f\right)\right\|_{L^{4}}\left\|\nabla\left(\chi_{8 d_{E}}^{c} h\right)\right\|_{L^{4}}+\left\|\chi_{4 d_{E}}^{c} J(f)\right\|_{L^{\infty}}\left\|\partial_{1} \nabla\left(\chi_{8 d_{E}}^{c} h\right)\right\|_{L^{2}} \\
& \lesssim\left\{\begin{array}{l}
d_{E}^{-\frac{3}{2}}\left(\frac{\nu s}{\rho}\right)^{\frac{1}{2}}\left\|R_{\nu} f\right\|_{X_{I B, 1}^{\left(0, \frac{\rho}{s}\right)}} \\
\|f\|_{X_{I I, 1}^{(0,0)}}
\end{array}\right\}\left\|\nabla\left(\chi_{8 d_{E}}^{c} h\right)\right\|_{W^{1,2} .}
\end{aligned}
$$

By using (3.13) and $\left\|B\left(f, \chi_{8 d_{E}}^{c} h\right)\right\|_{L^{1}} \leq\left\|\chi_{4 d_{E}}^{c} J(f)\right\|_{L^{4}}\left\|\nabla\left(\chi_{8 d_{E}}^{c} h\right)\right\|_{L^{4 / 3}}$ the $L^{1}$ norm is estimated as

$$
\left\|\varphi_{I}^{\left(0, \frac{\theta}{t}\right)} \Phi_{I, 3}^{(\nu)}[f, h](t-s)\right\|_{L^{1}} \lesssim\left\|B\left(f, \chi_{8 d_{E}}^{c} h\right)\right\|_{L^{1}} \lesssim\left\{\begin{array}{l}
d_{E}^{-1}\left(\frac{\nu s}{\rho}\right)^{\frac{1}{2}}\left\|R_{\nu} f\right\|_{X_{I B, 0}^{(0,0)}} \\
\|f\|_{X_{I I, 0}^{(0,0)}}
\end{array}\right\}\left\|\nabla\left(\chi_{8 d_{E}}^{c} h\right)\right\|_{L^{\frac{4}{3}}} .
$$

Again Lemma 2.5 is used. Thus (3.66) has been proved for $j=0$. To complete the proof for the case $j=1$ we use the equality $\partial_{2} \Phi_{I, 3}^{(\nu)}[f, h]=-e^{\nu(t-s) \Delta_{D}} \partial_{2} B\left(f, \chi_{8 d_{E}}^{c} h\right)$. Then the above argument implies

$$
\begin{aligned}
\left\|\varphi_{I}^{\left(\mu, \frac{\theta}{t}\right)} \partial_{2} \mathcal{F}\left(\Phi_{I, 3}^{(\nu)}[f, h](t-s)\right)\right\|_{L_{\xi_{1}}^{2} L_{x_{2}}^{2}} & \lesssim\left\|\partial_{2} B\left(f, \chi_{8 d_{E}}^{c} h\right)\right\|_{L^{2}} \\
& \lesssim\left(\left\|\chi_{7 d_{E}}^{c} \partial_{2} J(f)\right\|_{L^{4}}+\left\|\chi_{4 d_{E}}^{c} J(f)\right\|_{L^{\infty}}\right)\left\|\nabla\left(\chi_{8 d_{E}}^{c} h\right)\right\|_{W^{1,2}} .
\end{aligned}
$$

Since $\left\|\chi_{4 d_{E}}^{c} J(f)\right\|_{L^{\infty}}$ is estimated from Lemma 2.5 as above, we focus on $\left\|\chi_{7 d_{E}}^{c} \partial_{2} J(f)\right\|_{L^{4}}$. If $f \in X_{I I, 1}^{(0,0)}$ then the desired estimate follows from Lemma 2.5. On the other hand, if $R_{\nu} f \in X_{I B, 1}^{(0, \rho / s)}$ then we have from the equality $\partial_{2} J_{1}(f)=-f+\partial_{1} J_{2}(f)$ and $\partial_{2} J_{2}(f)=-\partial_{1} J_{1}(f)$,

$$
\begin{aligned}
\left\|\chi_{7 d_{E}}^{c} \partial_{2} J(f)\right\|_{L^{4}} \leq\left\|\chi_{7 d_{E}}^{c} f\right\|_{L^{4}}+\left\|\chi_{4 d_{E}}^{c} J\left(\partial_{1} f\right)\right\|_{L^{4}} & \lesssim\left\|\chi_{4 d_{E}}^{c} f\right\|_{L^{2}}^{\frac{1}{2}}\left\|\nabla\left(\chi_{4 d_{E}}^{c} f\right)\right\|_{L^{2}}^{\frac{1}{2}}+\left\|\chi_{4 d_{E}}^{c} J\left(\partial_{1} f\right)\right\|_{L^{4}} \\
& \lesssim d_{E}^{-1} e^{-\frac{\rho}{\nu s} d_{E}^{2}}\left\|R_{\nu} f\right\|_{X_{I B, 1}^{\left(0, \frac{\rho}{s}\right)}}+d_{E}^{-1}\left(\frac{\nu s}{\rho}\right)^{\frac{1}{2}}\left\|R_{\nu} f\right\|_{X_{I B, 1}^{\left(0, \frac{\rho}{s}\right)}} .
\end{aligned}
$$

The proof is complete. 


\subsection{Estimates for solutions of heat-transport equations}

We consider the heat-transport equations with the homogeneous Neumann boundary condition:

$$
\left\{\begin{array}{rll}
\partial_{t} H-\nu \Delta H+u \cdot \nabla H=f & t>0, & x \in \mathbb{R}_{+}^{2}, \\
\partial_{2} H=0 & t>0, & x \in \partial \mathbb{R}_{+}^{2}, \\
\left.H\right|_{t=0}=0 & & x \in \mathbb{R}_{+}^{2} .
\end{array}\right.
$$

Here the velocity $u$ is given by

$$
u=J\left(\omega_{E}+R_{\frac{1}{\nu}} w_{B}+R_{\frac{1}{\nu}} w_{I B}+w_{I I}\right)
$$

for some $\omega_{E} \in L^{\infty}\left(0, T ; X_{E}^{(0, \theta)} \cap Y_{E}\right), w_{B} \in L^{\infty}\left(0, T ; X_{B}^{(0, \rho)}\right), w_{I B} \in L^{\infty}\left(0, T ; X_{I B, 1}^{(0, \rho)}\right)$, and $w_{I I} \in$ $L^{\infty}\left(0, T ; X_{I I, 1}^{(0, \theta)}\right)$. Fix $\omega_{E}$ and $\omega_{B}$, and we will use the notation $\left\|(t-\cdot)^{-\kappa} f\right\|_{L^{1}(0, t ; Z)}=\int_{0}^{t}(t-s)^{-\kappa}\|f(s)\|_{Z} \mathrm{~d} s$. Then for $w_{I}=\left(w_{I B}, w_{I I}\right)$ we set

$$
\begin{aligned}
& A_{1, \nu}\left(t, w_{I}\right)=\left\|\nabla J\left(\omega_{E}\right)\right\|_{L^{1}\left(0, t ; L^{\infty}\right)}+\nu^{-\frac{1}{4}}\left\|(t-\cdot)^{-\frac{1}{4}} \chi_{\left\{x_{2} \geq 4 d_{E}\right\}} \nabla J\left(R_{\frac{1}{\nu}} w_{B}+R_{\frac{1}{\nu}} w_{I B}+w_{I I}\right)\right\|_{L^{1}\left(0, t ; L^{4}\right)}, \\
& A_{2, \nu}\left(t, w_{I}\right)=\left\|\nabla^{2} J\left(\omega_{E}\right)\right\|_{L^{1}\left(0, t ; L^{4}\right)}+\nu^{-\frac{1}{4}}\left\|(t-\cdot)^{-\frac{1}{4}} \chi_{\left\{x_{2} \geq 4 d_{E}\right\}} \nabla^{2} J\left(R_{\frac{1}{\nu}} w_{B}+R_{\frac{1}{\nu}} w_{I B}+w_{I I}\right)\right\|_{L^{1}\left(0, t ; L^{2}\right)}, \\
& A_{3, \nu}\left(t, w_{I}\right)=t\|u\|_{L^{\infty}\left(0, t ; L^{\infty}\right)}, \\
& A_{4, \nu}\left(t, w_{I}\right)=e^{-\frac{1}{32 \nu t} d_{E}^{2}}\left(\left\|\chi_{\left\{4 d_{E} \leq x_{2} \leq 5 d_{E}\right\}} \nabla u_{2}\right\|_{L^{\infty}\left(0, t ; L^{2}\right)}+\|u\|_{L^{\infty}\left(0, t ; L^{\infty}\right)}+1\right) .
\end{aligned}
$$

Proposition 3.11 Let $T \in\left(0, d_{E}\right)$. Assume that $f \in L^{\infty}\left(0, T ; W^{2,1}\left(\mathbb{R}_{+}^{2}\right) \cap W^{2,2}\left(\mathbb{R}_{+}^{2}\right)\right)$ and

$$
\begin{aligned}
& \cup_{0<t<T} \operatorname{supp} f(t) \subset\left\{x \in \mathbb{R}_{+}^{2} \mid x_{2} \geq m d_{E}\right\} \quad \text { for some } 12 \leq m \leq 32, \\
& \sup _{0<t<T}\|u(t)\|_{L^{\infty}} \leq \frac{d_{E}}{T}, \quad \sum_{j=2}^{4} \sup _{0<t<T} A_{j, \nu}\left(t, w_{I}\right) \leq 1 .
\end{aligned}
$$

Then there is $\delta>0$ independent of $\nu \in\left(0, d_{E}^{2}\right)$ and $d_{E}$ such that if $\sup _{0<t<T} A_{1, \nu}\left(t, w_{I}\right) \leq \delta$ then the solution $H \in C\left([0, T) ; L^{1}\left(\mathbb{R}_{+}^{2}\right) \cap L^{\infty}\left(\mathbb{R}_{+}^{2}\right)\right)$ of (HT) satisfies the following estimates for $0<t<T$.

$$
\begin{array}{rlrl}
\|H(t)\|_{L^{p}} & \lesssim \nu^{-\frac{1}{q}+\frac{1}{p}}\left\|(t-\cdot)^{-\frac{1}{q}+\frac{1}{p}} f\right\|_{L^{1}\left(0, t ; L^{q}\right)} & & 1 \leq q \leq p \leq \infty, \\
\left\|\chi_{\left\{x_{2} \leq(m-4) d_{E}\right\}} H(t)\right\|_{L^{p}} & \lesssim e^{-\frac{1}{8 \nu t} d_{E}^{2}}\|f\|_{L^{1}\left(0, t ; L^{1}\right)} & & 1 \leq p \leq \infty, \\
\left\|\chi_{\left\{x_{2} \leq(m-10) d_{E}\right\}} \nabla H(t)\right\|_{L^{p}} & \lesssim e^{-\frac{1}{16 \nu t} d_{E}^{2}}\|f\|_{L^{1}\left(0, t ; L^{1}\right)} & & 1<p<\infty, \\
\left\|\chi_{\left\{x_{2} \leq(m-10) d_{E}\right\}} \nabla^{2} H\right\|_{L^{q}\left(0, t ; L^{p}\right)} \lesssim e^{-\frac{1}{16 \nu t} d_{E}^{2}}\|f\|_{L^{1}\left(0, t ; L^{1}\right)} & & 1<p, q<\infty .
\end{array}
$$

Moreover, if $4 / 3 \leq p \leq 4$ and $1 \leq q \leq p$ then

$$
\begin{aligned}
\left\|\nabla\left(\chi_{4 d_{E}}^{c} H(t)\right)\right\|_{L^{p}} & \lesssim \nu^{-\frac{1}{q}+\frac{1}{p}}\left\|(t-\cdot)^{-\frac{1}{q}+\frac{1}{p}} \nabla f\right\|_{L^{1}\left(0, t ; L^{q}\right)}+\|f\|_{L^{1}\left(0, t ; L^{1}\right)}, \\
\left\|\nabla^{2}\left(\chi_{4 d_{E}}^{c} H(t)\right)\right\|_{L^{2}} & \lesssim\left\|\nabla^{2} f\right\|_{L^{1}\left(0, t ; L^{2}\right)}+\|\nabla f\|_{L^{1}\left(0, t ; L^{4}\right)}+\|f\|_{L^{1}\left(0, t ; L^{1}\right)} .
\end{aligned}
$$

Proof. Let $P_{u}^{(\nu)}(t, s)$ be the evolution operator associated with (HT). Since $H(t)=\int_{0}^{t} P_{u}^{(\nu)}(t, s) f(s) \mathrm{d} s$ the estimate (3.70) follows from (7.4) in Lemma 7.2. Next we prove (3.71). If $x_{2} \leq(m-4) d_{E}$ and $y_{2} \geq m d_{E}$, then we have from (3.69),

$$
|x-y|-\int_{s}^{t}\|u(\tau)\|_{L^{\infty}} \mathrm{d} \tau \geq|x-y|-T \sup _{0<t<T}\|u(t)\|_{L^{\infty}} \geq \frac{|x-y|}{2}+d_{E}
$$


for $0 \leq s<t \leq T$. Thus (7.6) yields $0 \leq P_{u}^{(\nu)}(t, x ; s, y) \lesssim(\nu(t-s))^{-1} \exp \left(-\frac{d_{E}^{2}}{4 \nu(t-s)}-\frac{|x-y|^{2}}{16 \nu(t-s)}\right)$ for $x_{2} \leq(m-4) d_{E}, y_{2} \geq m d_{E}$, and $0 \leq s<t \leq T$. Hence the Young inequality implies

$$
\left\|\chi_{\left\{x_{2} \leq(m-4) d_{E}\right\}} H(t)\right\|_{L^{p}} \lesssim \int_{0}^{t} \frac{1}{(\nu(t-s))^{1-\frac{1}{p}}} e^{-\frac{d_{E}^{2}}{4 \nu(t-s)}}\|f(s)\|_{L^{1}} \mathrm{~d} s \lesssim d_{E}^{-2\left(1-\frac{1}{p}\right)} e^{-\frac{1}{6 \nu t} d_{E}^{2}}\|f\|_{L^{1}\left(0, t ; L^{1}\right)},
$$

which proves (3.71) by $0<\nu<d_{E}^{2}$ and $0<t<d_{E}$. To show (3.77) we use the formula

$$
H(t)=-\int_{0}^{t} \partial_{1} e^{\nu(t-s) \Delta_{N}} u_{1} H \mathrm{~d} s-\int_{0}^{t} \partial_{2} e^{\nu(t-s) \Delta_{D}} u_{2} H \mathrm{~d} s+\int_{0}^{t} e^{\nu(t-s) \Delta_{N}} f \mathrm{~d} s=\sum_{i=1}^{3} H_{i}(t) .
$$

We decompose $H_{1}$ as $H_{1}=H_{1,1}+H_{1,2}$, where $H_{1,1}(t)=-\int_{0}^{t} \partial_{1} e^{\nu(t-s) \Delta_{N}}\left(u_{1} H \chi_{\left\{x_{2} \leq(m-4) d_{E}\right\}}\right) \mathrm{d} s$ and $H_{1,2}(t)=-\int_{0}^{t} \partial_{1} e^{\nu(t-s) \Delta_{N}}\left(u_{1} H \chi_{\left\{x_{2} \geq(m-4) d_{E}\right\}}\right) \mathrm{d} s$. By the maximal regularity we have for $1<p, q<\infty$, $\left\|\nabla H_{1,1}\right\|_{L^{q}\left(0, t ; L^{p}\right)} \lesssim \nu^{-1}\left\|u_{1} H \chi_{\left\{x_{2} \leq(m-4) d_{E}\right\}}\right\|_{L^{q}\left(0, t ; L^{p}\right)} \lesssim(\nu t)^{-1} A_{3, \nu}\left(t, w_{I}\right)\left\|H \chi_{\left\{x_{2} \leq(m-4) d_{E}\right\}}\right\|_{L^{q}\left(0, t ; L^{p}\right)}$, and thus, (3.69) and (3.71) yield $\left\|\nabla H_{1,1}\right\|_{L^{q}\left(0, t ; L^{p}\right)} \lesssim e^{-\frac{1}{12 \nu t} d_{E}^{2}}\|f\|_{L^{1}\left(0, t ; L^{1}\right)}$. On the other hand, since $|x-y| \geq$ $|x-y| / 2+d_{E}$ if $x_{2} \leq(m-6) d_{E}$ and $y_{2} \geq(m-4) d_{E}$ we have as in the proof of (3.71), by using (3.70),

$$
\left\|\chi_{\left\{x_{2} \leq(m-6) d_{E}\right\}} \nabla H_{1,2}(t)\right\|_{L^{p}} \lesssim \int_{0}^{t} \frac{e^{-\frac{1}{4 \nu(t-s)} d_{E}^{2}}\left\|u_{1} H\right\|_{L^{1}}}{(\nu(t-s))^{2-\frac{1}{p}}} \mathrm{~d} s \lesssim d_{E}^{-2\left(2-\frac{1}{p}\right)} e^{-\frac{1}{6 \nu t} d_{E}^{2}} A_{3, \nu}\left(t, w_{I}\right)\|f\|_{L^{1}\left(0, t ; L^{1}\right)},
$$

which implies $\left\|\chi_{\left\{x_{2} \leq(m-6) d_{E}\right\}} \nabla H_{1,2}\right\|_{L^{q}\left(0, t ; L^{p}\right)} \lesssim e^{-\frac{1}{8 \nu t} d_{E}^{2}}\|f\|_{L^{1}\left(0, t ; L^{1}\right)}$. The term $H_{2}$ is estimated similarly and the details are omitted. As for $H_{3}$, the representation of the heat kernel and (3.68) lead to $\left\|\chi_{\left\{x_{2} \leq(m-4) d_{E}\right\}} \nabla H_{3}(t)\right\|_{L^{p}} \lesssim e^{-\frac{1}{8 \nu t} d_{E}^{2}}\|f\|_{L^{1}\left(0, t ; L^{1}\right)}$. Collecting these, we get

$$
\left\|\chi_{\left\{x_{2} \leq(m-6) d_{E}\right\}} \nabla H\right\|_{L^{q}\left(0, t ; L^{p}\right)} \lesssim e^{-\frac{1}{12 \nu t} d_{E}^{2}}\|f\|_{L^{1}\left(0, t ; L^{1}\right)} \quad 1<p, q<\infty .
$$

To prove (3.72) and (3.73) we decompose $H$ as $H=\sum_{i=1}^{3} H_{i}^{\prime}$, where

$\sum_{i=1}^{3} H_{i}^{\prime}(t)=-\int_{0}^{t} e^{\nu(t-s) \Delta_{N}} u \cdot \nabla\left(H \chi_{(m-7) d_{E}}\right) \mathrm{d} s-\int_{0}^{t} e^{\nu(t-s) \Delta_{N}} u \cdot \nabla\left(H \chi_{(m-7) d_{E}}^{c}\right) \mathrm{d} s+\int_{0}^{t} e^{\nu(t-s) \Delta_{N}} f \mathrm{~d} s$.

The first term is estimated from (3.71) and (3.77) as

$$
\begin{aligned}
\left\|\chi_{\left\{x_{2} \leq(m-9) d_{E}\right\}} \nabla H_{1}^{\prime}(t)\right\|_{L^{p}} \lesssim \int_{0}^{t} \frac{\left\|u \cdot \nabla\left(H \chi_{(m-7) d_{E}}\right)\right\|_{L^{p}}}{(\nu(t-s))^{\frac{1}{2}}} \mathrm{~d} s & \lesssim \frac{t^{\frac{1}{4}}}{\nu^{\frac{1}{2}}}\|u\|_{L^{\infty}\left(0, t ; L^{\infty}\right)}\left\|\nabla\left(H \chi_{(m-7) d_{E}}\right)\right\|_{L^{4}\left(0, t ; L^{p}\right)} \\
& \lesssim e^{-\frac{1}{16 \nu t} d_{E}^{2}} A_{3, \nu}\left(t, w_{I}\right)\|f\|_{L^{1}\left(0, t ; L^{1}\right)},
\end{aligned}
$$

and by the maximal regularity together with (3.71) and (3.77) we also have for $1<p, q<\infty$,

$$
\left\|\nabla^{2} H_{1}^{\prime}\right\|_{L^{q}\left(0, t ; L^{p}\right)} \lesssim \nu^{-1}\left\|u \cdot \nabla\left(H \chi_{(m-7) d_{E}}\right)\right\|_{L^{q}\left(0, t ; L^{p}\right)} \lesssim A_{3, \nu}\left(t, w_{I}\right) e^{-\frac{1}{16 \nu t} d_{E}^{2}}\|f\|_{L^{1}\left(0, t ; L^{1}\right)} .
$$

By using $u \cdot \nabla\left(H \chi_{(m-7) d_{E}}\right)=\nabla \cdot\left(u H \chi_{(m-7) d_{E}}\right)$ and (3.70) the term $H_{2}^{\prime}$ is estimated as

$$
\left\|\chi_{\left\{x_{2} \leq(m-9) d_{E}\right\}} \nabla H_{2}^{\prime}(t)\right\|_{L^{p}} \lesssim \int_{0}^{t} \frac{e^{-\frac{1}{4 \nu(t-s)} d_{E}^{2}}}{(\nu(t-s))^{\frac{3}{2}-\frac{1}{p}}}\|u H\|_{L^{1}} \mathrm{~d} s \lesssim d_{E}^{-2\left(\frac{3}{2}-\frac{1}{p}\right)} e^{-\frac{1}{6 \nu t} d_{E}^{2}} A_{3, \nu}\left(t, w_{I}\right)\|f\|_{L^{1}\left(0, t ; L^{1}\right)}
$$


and

$$
\left\|\chi_{\left\{x_{2} \leq(m-9) d_{E}\right\}} \nabla^{2} H_{2}^{\prime}(t)\right\|_{L^{p}} \lesssim \int_{0}^{t} \frac{e^{-\frac{1}{4 \nu(t-s)} d_{E}^{2}}}{(\nu(t-s))^{\frac{5}{2}-\frac{1}{p}}}\|u H\|_{L^{1}} \mathrm{~d} s \lesssim d_{E}^{-2\left(\frac{5}{2}-\frac{1}{p}\right)} e^{-\frac{1}{6 \nu t} d_{E}^{2}} A_{3, \nu}\left(t, w_{I}\right)\|f\|_{L^{1}\left(0, t ; L^{1}\right)} .
$$

It is easy to estimate $\left\|\chi_{\left\{x_{2} \leq(m-9) d_{E}\right\}} \nabla H_{3}^{\prime}(t)\right\|_{L^{p}}$ and $\left\|\chi_{\left\{x_{2} \leq(m-4) d_{E}\right\}} \nabla^{2} H_{3}^{\prime}\right\|_{L^{2}\left(0, t ; L^{2}\right)}$, so we omit the details. The proof of (3.72) and (3.73) is complete. To show (3.74) set $H_{E}=H \chi_{4 d_{E}}^{c}$. Then $\nabla H_{E}$ satisfies

$$
\partial_{t} \nabla H_{E}-\nu \Delta \nabla H_{E}+u \cdot \nabla \nabla H_{E}=-\nabla u \cdot \nabla H_{E}-\nabla\left(2 \nu \partial_{2} \chi_{4 d_{E}}^{c} \partial_{2} H+\nu \partial_{2}^{2} \chi_{4 d_{E}}^{c} H-u_{2} H \partial_{2} \chi_{4 d_{E}}^{c}\right)+\nabla f
$$

with $\partial_{2} \nabla H_{E}=0$ on $\partial_{2} \mathbb{R}_{+}^{2}$. By using (7.4), (3.71), (3.72), and (3.73) we have for $4 / 3 \leq p<\infty$,

$$
\begin{aligned}
& \left\|\nabla H_{E}(t)\right\|_{L^{p}} \lesssim\left\|\nabla J\left(\omega_{E}\right) \cdot \nabla H_{E}\right\|_{L^{1}\left(0, t ; L^{p}\right)}+\int_{0}^{t} \frac{\left\|\nabla J\left(R_{\frac{1}{\nu}} w_{B}+R_{\frac{1}{\nu}} w_{I B}+w_{I I}\right) \cdot \nabla H_{E}\right\|_{L^{\frac{4 p}{4} p}}}{d s} \mathrm{~d} s \\
& +\nu\left\|\nabla\left(\partial_{2} \chi_{4 d_{E}}^{c} \partial_{2} H\right)\right\|_{L^{1}\left(0, t ; L^{p}\right)}+\nu\left\|\nabla\left(\partial_{2}^{2} \chi_{4 d_{E}}^{c} H\right)\right\|_{L^{1}\left(0, t ; L^{p}\right)}+\int_{0}^{t} \frac{\left\|\nabla\left(u_{2} H \partial_{2} \chi_{4 d_{E}}^{c}\right)\right\|_{L^{\frac{4}{3}}}}{(\nu(t-s))^{\frac{3}{4}-\frac{1}{p}}} \frac{\|\nabla f\|_{L^{q}}}{(\nu(t-s))^{\frac{1}{q}-\frac{1}{p}}} \mathrm{~d} s \\
& \lesssim A_{1, \nu}\left(t, w_{I}\right) \sup _{0<s<t}\left\|\nabla H_{E}(s)\right\|_{L^{p}}+A_{4, \nu}\left(t, w_{I}\right)\|f\|_{L^{1}\left(0, t ; L^{1}\right)}+\left\|(\nu(t-\cdot))^{-\frac{1}{q}+\frac{1}{p}} \nabla f\right\|_{L^{1}\left(0, t ; L^{q}\right)} .
\end{aligned}
$$

Thus (3.74) follows if $\sup _{0<t<T} A_{1, \nu}\left(t, w_{I}\right) \leq \delta \ll 1$. The estimate of $\nabla^{2} H_{E}$ is obtained from the equation

$$
\begin{aligned}
& \partial_{t} \nabla^{2} H_{E}-\nu \Delta \nabla^{2} H_{E}+u \cdot \nabla \nabla^{2} H_{E} \\
= & -\nabla u \cdot \nabla \nabla H_{E}-\nabla\left(\nabla u \cdot \nabla H_{E}\right)-\nabla^{2}\left(2 \nu \partial_{2} \chi_{4 d_{E}}^{c} \partial_{2} H+\nu \partial_{2}^{2} \chi_{4 d_{E}}^{c} H-u_{2} H \partial_{2} \chi_{4 d_{E}}^{c}\right)+\nabla^{2} f
\end{aligned}
$$

with $\partial_{2} \nabla^{2} H_{E}=0$ on $\partial \mathbb{R}_{+}^{2}$. Then from (7.4), (7.5), and the Hölder inequality, we have

$$
\begin{aligned}
& \left\|\nabla^{2} H_{E}(t)\right\|_{L^{2}} \lesssim A_{1, \nu}\left(t, w_{I}\right) \sup _{0<s<t}\left\|\nabla^{2} H_{E}(s)\right\|_{L^{2}}+A_{2, \nu}\left(t, w_{I}\right) \sup _{0<s<t}\left\|\nabla H_{E}(s)\right\|_{L^{4}}+\left\|\nabla^{2} f\right\|_{L^{1}\left(0, t ; L^{2}\right)} \\
& \quad+\nu^{\frac{1}{2}}\left\|\nabla\left(\partial_{2} \chi_{4 d_{E}}^{c} \partial_{2} H\right)\right\|_{L^{2}\left(0, t ; L^{2}\right)}+\nu^{\frac{1}{2}}\left\|\nabla\left(\partial_{2}^{2} \chi_{4 d_{E}}^{c} H\right)\right\|_{L^{2}\left(0, t ; L^{2}\right)}+\nu^{-\frac{1}{2}}\left\|\nabla\left(u_{2} H \partial_{2} \chi_{4 d_{E}}^{c}\right)\right\|_{L^{2}\left(0, t ; L^{2}\right)} .
\end{aligned}
$$

From (3.71), (3.72), (3.73), and (3.77), it is not difficult to see

$$
\begin{aligned}
& \nu^{\frac{1}{2}}\left\|\nabla\left(\partial_{2} \chi_{4 d_{E}}^{c} \partial_{2} H\right)\right\|_{L^{2}\left(0, t ; L^{2}\right)}+\nu^{\frac{1}{2}}\left\|\nabla\left(\partial_{2}^{2} \chi_{4 d_{E}}^{c} H\right)\right\|_{L^{2}\left(0, t ; L^{2}\right)}+\nu^{-\frac{1}{2}}\left\|\nabla\left(u_{2} H \partial_{2} \chi_{4 d_{E}}^{c}\right)\right\|_{L^{2}\left(0, t ; L^{2}\right)} \\
\lesssim & A_{4, \nu}\left(t, w_{I}\right)\|f\|_{L^{1}\left(0, t ; L^{1}\right)},
\end{aligned}
$$

Hence (3.75) follows from (3.74) with $p=4$ if $\sup _{0<t<T} A_{1, \nu}\left(t, w_{I}\right) \leq \delta \ll 1$. This completes the proof.

In the construction of $\omega_{I}$ we also need the estimates for solutions of the equation

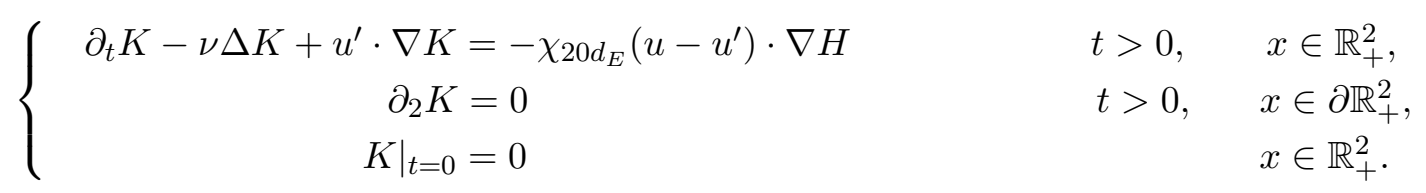

Here $H$ is the solution of (HT) and the velocity fields $u, u^{\prime}$ are given as

$$
u=J\left(\omega_{E}+R_{\frac{1}{\nu}} w_{B}+R_{\frac{1}{\nu}} w_{I B}+w_{I I}\right), \quad u^{\prime}=J\left(\omega_{E}+R_{\frac{1}{\nu}} w_{B}+R_{\frac{1}{\nu}} w_{I B}^{\prime}+w_{I I}^{\prime}\right)
$$

for some $\omega_{E} \in L^{\infty}\left(0, T ; X_{E}^{(0, \theta)}\right), w_{B} \in L^{\infty}\left(0, T ; X_{B}^{(0, \rho)}\right), w_{I B}, w_{I B}^{\prime} \in L^{\infty}\left(0, T ; X_{I B, 1}^{(0, \rho)}\right)$, and $w_{I I}, w_{I I}^{\prime} \in$ $L^{\infty}\left(0, T ; X_{I I, 1}^{(0, \theta)}\right)$. For $f_{I}=\left(f_{I B}, f_{I I}\right)$ we set $\left\|f_{I}\right\|_{X_{I, 0}^{(\mu, \rho, \theta)}}=\left\|f_{I B}\right\|_{X_{I B, 0}^{(\mu, \rho)}}+\left\|f_{I I}\right\|_{X_{I I, 0}^{(\mu, \theta)}}$. 
Proposition 3.12 Let $T \in\left(0, d_{E}\right)$. Assume that $\sup _{0<t<T} A_{3, \nu}\left(t, w_{I}^{\prime}\right) \leq 1$ and that $H$ satisfies the estimates in Proposition 3.11 with $m=32$. Let $K \in C\left([0, T) ; L^{1}\left(\mathbb{R}_{+}^{2}\right) \cap L^{\infty}\left(\mathbb{R}_{+}^{2}\right)\right)$ be the solution of (3.78). If $4 / 3 \leq p<\infty$ then

$$
\begin{aligned}
\|K(t)\|_{L^{p}} & \lesssim e^{-\frac{1}{16 \nu t} d_{E}^{2}}\|f\|_{L^{1}\left(0, t ; L^{1}\right)} \sup _{0<s<t}\left\|w_{I}(s)-w_{I}^{\prime}(s)\right\|_{X_{I, 0}^{(0,0,0)}}, \\
\|\nabla K(t)\|_{L^{p}} & \lesssim e^{-\frac{1}{32 \nu t} d_{E}^{2}}\|f\|_{L^{1}\left(0, t ; L^{1}\right)} \sup _{0<s<t}\left\|w_{I}(s)-w_{I}^{\prime}(s)\right\|_{X_{I, 0}^{(0,0,0)} .}
\end{aligned}
$$

Proof. From (7.4) we have

$$
\|K(t)\|_{L^{p}} \lesssim \int_{0}^{t} \frac{\left\|\chi_{20 d_{E}}\left(u-u^{\prime}\right) \cdot \nabla H\right\|_{L^{\frac{4 p}{4+p}}}}{(\nu(t-s))^{\frac{1}{4}}} \mathrm{~d} s \lesssim \nu^{-\frac{1}{4}} t^{\frac{1}{2}}\left\|u-u^{\prime}\right\|_{L^{\infty}\left(0, t ; L^{4}\right)}\left\|\chi_{20 d_{E}} \nabla H\right\|_{L^{4}\left(0, t ; L^{p}\right)} .
$$

Thus (3.80) follows from (3.77) and Lemma 2.5. Next we use the formula

$$
K(t)=-\int_{0}^{t} e^{\nu(t-s) \Delta_{N}} u^{\prime} \cdot \nabla K \mathrm{~d} s-\int_{0}^{t} e^{\nu(t-s) \Delta_{N}} \chi_{20 d_{E}}\left(u-u^{\prime}\right) \cdot \nabla H \mathrm{~d} s=K_{1}(t)+K_{2}(t) .
$$

Since $\nabla \cdot u^{\prime}=0$ the first term is estimated from the maximal regularity and (3.80) as

$$
\begin{aligned}
\left\|\nabla K_{1}\right\|_{L^{q}\left(0, t ; L^{p}\right)} & \lesssim \nu^{-1}\left\|u^{\prime} K\right\|_{L^{q}\left(0, t ; L^{p}\right)} \lesssim \nu^{-1}\left\|u^{\prime}\right\|_{L^{\infty}\left(0, t ; L^{\infty}\right)}\|K\|_{L^{q}\left(0, t ; L^{p}\right)} \\
& \lesssim A_{3, \nu}\left(t, w_{I}^{\prime}\right) e^{-\frac{1}{24 \nu t} d_{E}^{2}}\|f\|_{L^{1}\left(0, t ; L^{1}\right)} \sup _{0<s<T}\left\|w_{I}(s)-w_{I}^{\prime}(s)\right\|_{X_{I, 0}^{(0,0,0)}} .
\end{aligned}
$$

As for $K_{2}$, we have from (3.72),

$$
\begin{aligned}
\left\|\nabla K_{2}(t)\right\|_{L^{p}} \lesssim \int_{0}^{t} \frac{\left\|\chi_{20 d_{E}}\left(u-u^{\prime}\right) \cdot \nabla H\right\|_{L^{\frac{4 p}{4+p}}}}{(\nu(t-s))^{\frac{3}{4}}} \mathrm{~d} s & \lesssim\left\|u-u^{\prime}\right\|_{L^{\infty}\left(0, t ; L^{4}\right)} \int_{0}^{t} \frac{\left\|\chi_{20 d_{E}} \nabla H\right\|_{L^{p}}}{(\nu(t-s))^{\frac{3}{4}}} \mathrm{~d} s \\
& \lesssim e^{-\frac{1}{24 \nu t} d_{E}^{2}}\left\|u-u^{\prime}\right\|_{L^{\infty}\left(0, t ; L^{4}\right)}\|f\|_{L^{1}\left(0, t ; L^{1}\right)} .
\end{aligned}
$$

Again from the definition of $K_{1}$ we have $\left\|\nabla K_{1}(t)\right\|_{L^{p}} \lesssim\left\|u^{\prime}\right\|_{L^{\infty}\left(0, t ; L^{\infty}\right)}\left\|(\nu(t-\cdot))^{-1 / 2} \nabla K\right\|_{L^{1}\left(0, t ; L^{p}\right)}$. Then (3.83), (3.84), and Lemma 2.5 yield (3.81). The details are omitted here. This completes the proof.

The following proposition is used to verify the conditions in Proposition 3.11.

Proposition 3.13 Let $\omega_{E}$ be the solution of $\left(\mathrm{V}_{\mathrm{E}}\right)$ and let $T_{0}$ be the time in $(2.3)$. Then there is $T_{0}^{\prime} \in$ $\left(0, T_{0}\right)$ such that if $0<\nu<d_{E}^{5}, T \in\left(0, T_{0}^{\prime}\right]$, and

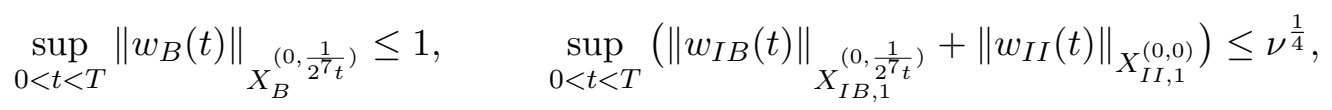

then we have for $u=J\left(\omega_{E}+R_{1 / \nu} w_{B}+R_{1 / \nu} w_{I B}+w_{I I}\right)$,

$$
\sup _{0<t<T}\|u(t)\|_{L^{\infty}} \leq \frac{d_{E}}{T}, \quad \sup _{0<t<T} A_{1, \nu}\left(t, w_{I}\right) \leq \delta, \quad \sum_{j=2}^{4} \sup _{0<t<T} A_{j, \nu}\left(t, w_{I}\right) \leq 1 .
$$

Here $\delta>0$ is the number in Proposition 3.11 and $T_{0}^{\prime}$ is taken so that $T_{0}^{\prime} \geq c^{\prime} d_{E}$ for some constant depending only on $\|b\|_{W^{4,1} \cap W^{4,2} \text {. }}$ 
Proof. The assertion follows from Lemma 2.5 and the definitions of $A_{i, \nu}\left(t, w_{I}\right)$. The requirement of $\nu<d_{E}^{5}$ is to ensure the lower bound $T_{0}^{\prime} \geq c^{\prime} d_{E}$, which comes from the term $A_{2, \nu}\left(t, w_{I}\right)$ and the application of (2.20). We skip the details. The proof is complete.

Let $H$ be the solution of (HT) with $u=J\left(\omega_{E}+R_{1 / \nu} w_{B}+R_{1 / \nu} w_{I B}+w_{I I}\right)$, and let $H^{\prime}$ be the solution of (HT) with $u^{\prime}=J\left(\omega_{E}+R_{1 / \nu} w_{B}+R_{1 / \nu} w_{I B}^{\prime}+w_{I I}^{\prime}\right)$ and $f$ replaced by $f^{\prime}$. Then we have

Proposition 3.14 Suppose that $f, f^{\prime} \in L^{\infty}\left(0, T_{0} ; W^{2,1}\left(\mathbb{R}_{+}^{2}\right) \cap W^{2,2}\left(\mathbb{R}_{+}^{2}\right)\right)$ and that $f, f^{\prime}$ satisfy (3.68) with $m=32$. Assume that $w_{B}, w_{I}=\left(w_{I B}, w_{I I}\right)$, and $w_{I}^{\prime}=\left(w_{I B}^{\prime}, w_{I I}^{\prime}\right)$ satisfy (3.85). Let $0<\nu<d_{E}^{5}$ and let $T_{0}^{\prime}>0$ be the number in Proposition 3.13. Then for $0<t \leq T_{0}^{\prime}$ and $4 / 3 \leq p \leq 2$ it follows that

$$
\begin{aligned}
& \left\|\chi_{\left\{x_{2} \leq 16 d_{E}\right\}}\left(H(t)-H^{\prime}(t)\right)\right\|_{L^{p}} \\
\lesssim & e^{-\frac{1}{32 \nu t} d_{E}^{2}}\left(\sup _{0<s<t}\left\|w_{I}(s)-w_{I}^{\prime}(s)\right\|_{X_{I, 0}^{(0,0,0)}}\left(\|\nabla f\|_{L^{1}\left(0, t ; L^{\frac{4}{3}}\right)}+\|f\|_{L^{1}\left(0, t ; L^{1}\right)}\right)+\left\|f-f^{\prime}\right\|_{L^{1}\left(0, t ; L^{1}\right)}\right), \\
& \left\|\nabla\left(\chi_{8 d_{E}}^{c} H(t)-\chi_{8 d_{E}}^{c} H^{\prime}(t)\right)\right\|_{L^{p}} \\
\lesssim & \nu^{-\frac{3}{4}+\frac{1}{p}} \sup _{0<s<t}\left\|w_{I}(s)-w_{I}^{\prime}(s)\right\|_{X_{I, 0}^{\left(0, \frac{1}{2^{7}}, 0\right)}}\left(\|\nabla f\|_{L^{1}\left(0, t ; W^{1,2}\right)}+\|\nabla f\|_{L^{1}\left(0, t ; L^{\frac{4}{3}}\right)}+\|f\|_{L^{1}\left(0, t ; L^{1}\right)}\right) \\
& +\left\|\nabla\left(f-f^{\prime}\right)\right\|_{L^{1}\left(0, t ; L^{p}\right)}+\left\|f-f^{\prime}\right\|_{L^{1}\left(0, t ; L^{1}\right)} .
\end{aligned}
$$

Proof. We decompose $H-H^{\prime}$ as $H-H^{\prime}=K+L$, where $K$ is the solution of (3.78) and $L$ solves

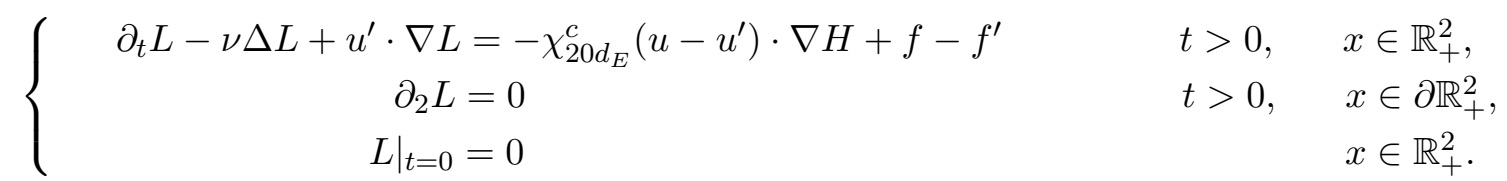

Then by applying Proposition 3.11 to (3.89) with $m=20$ we have

$$
\begin{aligned}
\left\|\chi_{\left\{x_{2} \leq 16 d_{E}\right\}} L(t)\right\|_{L^{p}} & \lesssim e^{-\frac{1}{8 \nu t} d_{E}^{2}}\left(\left\|\chi_{20 d_{E}}^{c}\left(u-u^{\prime}\right) \cdot \nabla H\right\|_{L^{1}\left(0, t ; L^{1}\right)}+\left\|f-f^{\prime}\right\|_{L^{1}\left(0, t ; L^{1}\right)}\right), \\
\left\|\nabla\left(\chi_{8 d_{E}}^{c} L(t)\right)\right\|_{L^{p}} \lesssim & \int_{0}^{t}(\nu(t-s))^{-\frac{3}{4}+\frac{1}{p}}\left\|\nabla\left(\chi_{20 d_{E}}^{c}\left(u-u^{\prime}\right) \cdot \nabla H\right)\right\|_{L^{\frac{4}{3}}} \mathrm{~d} s \\
& +\left\|\nabla\left(f-f^{\prime}\right)\right\|_{L^{1}\left(0, t ; L^{p}\right)}+\left\|\chi_{20 d_{E}}^{c}\left(u-u^{\prime}\right) \cdot \nabla H\right\|_{L^{1}\left(0, t ; L^{1}\right)}+\left\|f-f^{\prime}\right\|_{L^{1}\left(0, t ; L^{1}\right)}
\end{aligned}
$$

for $4 / 3 \leq p \leq 2$. The Hölder inequality, Lemma 2.5, and Proposition 3.11 imply

$$
\begin{aligned}
\left\|\chi_{20 d_{E}}^{c}\left(u(s)-u^{\prime}(s)\right) \cdot \nabla H(s)\right\|_{L^{1}} & \leq\left\|u(s)-u^{\prime}(s)\right\|_{L^{4}}\left\|\nabla\left(\chi_{8 d_{E}}^{c} H(s)\right)\right\|_{L^{\frac{4}{3}}} \\
& \lesssim\left\|w_{I}(s)-w_{I}^{\prime}(s)\right\|_{X_{I, 0}^{(0,0,0)}}\left(\|\nabla f\|_{L^{1}\left(0, s ; L^{\frac{4}{3}}\right)}+\|f\|_{L^{1}\left(0, s ; L^{1}\right)}\right),
\end{aligned}
$$

and

$$
\begin{aligned}
& \left\|\nabla\left(\chi_{20 d_{E}}^{c}\left(u(s)-u^{\prime}(s)\right) \cdot \nabla H(s)\right)\right\|_{L^{\frac{4}{3}}} \\
\leq & \left\|u(s)-u^{\prime}(s)\right\|_{L^{4}}\left\|\nabla\left(\chi_{20 d_{E}}^{c} \nabla H(s)\right)\right\|_{L^{2}}+\left\|\chi_{4 d_{E}}^{c} \nabla\left(u(s)-u^{\prime}(s)\right)\right\|_{L^{2}}\left\|\chi_{20 d_{E}}^{c} \nabla H(s)\right\|_{L^{4}} \\
\lesssim & \left\|w_{I}(s)-w_{I}^{\prime}(s)\right\|_{X_{I, 0}^{(0,0,0)}}\left(\left\|\nabla^{2} f\right\|_{L^{1}\left(0, s ; L^{2}\right)}+\|\nabla f\|_{L^{1}\left(0, s ; L^{4}\right)}+\|f\|_{L^{1}\left(0, s ; L^{1}\right)}\right) \\
& +\left(\left(\frac{\nu s}{d_{E}^{3} \rho}\right)^{\frac{1}{2}}\left\|w_{I B}(s)-w_{I B}^{\prime}(s)\right\|_{X_{I B, 0}^{(0, \rho)}}+\left\|w_{I I}(s)-w_{I I}^{\prime}(s)\right\|_{X_{I I, 0}^{(0,0)}}\right)\left(\|\nabla f\|_{L^{1}\left(0, s ; L^{4}\right)}+\|f\|_{L^{1}\left(0, s ; L^{1}\right)}\right) \\
\lesssim & \rho^{-\frac{1}{2}}\left\|w_{I}(s)-w_{I}^{\prime}(s)\right\|_{X_{I, 0}^{\left(0, \frac{\rho}{s}, 0\right)}}\left(\|\nabla f\|_{L^{1}\left(0, s ; W^{1,2}\right)}+\|f\|_{L^{1}\left(0, s ; L^{1}\right)}\right)
\end{aligned}
$$

for $0<\nu<d_{E}^{5}$. Take $\rho=2^{-7}$. Collecting these, we observe that $\left\|\chi_{\left\{x_{2} \leq 16 d_{E}\right\}} L(t)\right\|_{L^{p}}$ and $\left\|\nabla\left(\chi_{8 d_{E}}^{c} L(t)\right)\right\|_{L^{p}}$ are bounded from above by the right-hand side of (3.87) and (3.88), respectively. Since $K$ is already estimated in Proposition 3.12, the proof has been completed. 


\section{Construction of solutions}

In this section we construct the solution $\omega_{B}=R_{1 / \nu} w_{B}$ of $\left(\mathrm{V}_{\mathrm{B}_{\nu}}\right)$ and the solution $\omega_{I}=R_{1 / \nu} w_{I B}+w_{I I}$ of $\left(\mathrm{V}_{\mathrm{I}_{\nu}}\right)$ by solving the associated integral equations. Lemmas 2.1, 2.2, and 2.3 imply the integral equation for $w_{B}$ such as

$$
w_{B}(t)=\int_{0}^{t} \Lambda_{B}^{(\nu)}\left(t, s, w_{B}\right) \mathrm{d} s+\int_{0}^{t} F_{B}^{(\nu)}(t, s) \mathrm{d} s,
$$

where

$$
\begin{aligned}
\Lambda_{B}^{(\nu)}\left(t, s, w_{B}\right)= & \sum_{i=1}^{2} \Phi_{B, i}^{(\nu)}\left[R_{\nu} \omega_{E}+w_{B}, w_{B}\right](t-s)+\sum_{i=1}^{2} \phi_{t^{\frac{1}{2}}} \Psi_{i}^{(\nu)}\left[R_{\nu} \omega_{E}+w_{B}, w_{B}\right](t-s) \\
& +\sum_{i=1}^{2} \phi_{t^{\frac{1}{2}}}^{c} \Upsilon_{i}^{(\nu)}\left[R_{\nu} \omega_{E}+w_{B}, w_{B}\right](s), \\
F_{B}^{(\nu)}(t, s)= & \Phi_{B, 2}\left[R_{\nu} \omega_{E}, R_{\nu} \omega_{E}\right](t-s)+\Upsilon_{2}^{(\nu)}\left[R_{\nu} \omega_{E}, R_{\nu} \omega_{E}\right](s) .
\end{aligned}
$$

Precisely speaking, $\Phi_{B, i}^{(\nu)}\left[R_{\nu} \omega_{E}+w_{B}, w_{B}\right]$ should be expressed as $\Phi_{B, i}^{(\nu)}\left[R_{\nu} \omega_{E}(s)+w_{B}(s), w_{B}(s)\right]$, but we will use the abbreviated style for simplicity of notations. The similar remark is added for the other terms.

The system for the remainder part $w_{I}=\left(w_{I B}, w_{I I}\right)$ is described as

$$
w_{I B}(t)=\int_{0}^{t} \Lambda_{I B}^{(\nu)}\left(t, s, w_{I}\right) \mathrm{d} s+\int_{0}^{t} F_{I B}^{(\nu)}(t, s) \mathrm{d} s, \quad w_{I I}(t)=\int_{0}^{t} \Lambda_{I I}^{(\nu)}\left(t, s, w_{I}\right) \mathrm{d} s+\int_{0}^{t} F_{I I}^{(\nu)}(t, s) \mathrm{d} s,
$$

where, by setting $\omega=\omega_{E}+R_{1 / \nu} w_{B}+R_{1 / \nu} w_{I B}+w_{I I}$, each term is defined by

$$
\begin{aligned}
\Lambda_{I B}^{(\nu)}\left(t, s, w_{I}\right)= & \sum_{i=1}^{2} \Phi_{B, i}^{(\nu)}\left[R_{\nu} \omega, w_{I B}\right](t-s)+\sum_{i=1}^{2} \Phi_{B, i}^{(\nu)}\left[w_{I B}+R_{\nu} w_{I I}, w_{B}\right](t-s) \\
& +\Phi_{B, 2}^{(\nu)}\left[R_{\nu} \omega, R_{\nu} w_{I I}\right](t-s)+\Phi_{B, 2}^{(\nu)}\left[w_{I B}+R_{\nu} w_{I I}, R_{\nu} \omega_{E}\right](t-s) \\
& +\sum_{i=1}^{2} \phi_{t^{\frac{1}{2}}} \Psi_{i}^{(\nu)}\left[R_{\nu} \omega, w_{I B}+R_{\nu} w_{I I}\right](t-s)+\sum_{i=1}^{2} \phi_{t^{\frac{1}{2}}} \Psi_{i}^{(\nu)}\left[w_{I B}+R_{\nu} w_{I I}, R_{\nu} \omega_{E}+w_{B}\right](t-s) \\
& +\sum_{i=1}^{2} \phi_{t^{\frac{1}{2}}}^{c} \Upsilon_{i}^{(\nu)}\left[R_{\nu} \omega, w_{I B}+R_{\nu} w_{I I}\right](s)+\sum_{i=1}^{2} \phi_{t^{\frac{1}{2}}}^{c} \Upsilon_{i}^{(\nu)}\left[w_{I B}+R_{\nu} w_{I I}, R_{\nu} \omega_{E}+w_{B}\right](s), \quad(4.5) \\
F_{I B}^{(\nu)}(t, s)= & \Phi_{B, 2}^{(\nu)}\left[w_{B}, R_{\nu} \omega_{E}\right](t-s)-\nu R_{\nu} e^{\nu(t-s) \Delta_{N}}\left(J_{1}\left(\Delta \omega_{E}\right) \mathcal{H}_{\left\{X_{2}=0\right\}}^{1}\right) \\
& +\sum_{i=1}^{2} \Upsilon_{i}^{(\nu)}\left[w_{B}, R_{\nu} \omega_{E}\right](s)-\nu^{2} R_{\nu} \int_{0}^{s} \Xi G(\nu(s-\tau)) \star\left(\Delta \omega_{E}-J_{1}\left(\Delta \omega_{E}\right) \mathcal{H}_{\left\{X_{2}=0\right\}}^{1}\right) \mathrm{d} \tau, \\
\Lambda_{I I}^{(\nu)}\left(t, s, w_{I}\right)= & \Phi_{I}^{(\nu)}\left[R_{\frac{1}{\nu}} w_{I B}+w_{I I}, \omega_{E}\right](t-s)+\Phi_{I, 1}^{(\nu)}\left[\omega, w_{I I}\right](t-s)+\sum_{i=2}^{3} \Phi_{I, i}^{(\nu)}\left[\omega, H^{(\nu)}\left[w_{I}\right]\right](t-s), \quad(4.6) \\
F_{I I}^{(\nu)}(t, s)= & \Phi_{I}^{(\nu)}\left[R_{\frac{1}{\nu}} w_{B}, \omega_{E}\right](t-s)+\nu e^{\nu(t-s) \Delta_{N}} \Delta \omega_{E} .
\end{aligned}
$$

Here $H^{(\nu)}\left[w_{I}\right]$ is defined by

$$
H^{(\nu)}\left[w_{I}\right](t)=-\int_{0}^{t} P_{u}^{(\nu)}(t, s)\left(B\left(R_{\frac{1}{\nu}} w_{B}+R_{\frac{1}{\nu}} w_{I B}+w_{I I}, \omega_{E}\right)-\nu \Delta \omega_{E}\right) \mathrm{d} s,
$$


where $P_{u}^{(\nu)}(t, s)$ is the propagator for the heat-transport equations (HT) with

$$
u=J(\omega)=J\left(\omega_{E}+R_{\frac{1}{\nu}} w_{B}+R_{\frac{1}{\nu}} w_{I B}+w_{I I}\right) .
$$

The use of $H^{(\nu)}\left[w_{I}\right]$ rather than $w_{I I}$ in (4.6) is essential in order to overcome the difficulty arising from the lack of the analyticity in the region away from the boundary.

\subsection{Solutions of $\left(\mathrm{V}_{\mathrm{B}_{\nu}}\right)$}

To apply the ACK theorem to (4.1) we set the iteration sequence $\left\{w_{B}^{(k)}\right\}_{k=0}^{\infty}$ by

$$
w_{B}^{(0)}(t)=\int_{0}^{t} F_{B}^{(\nu)}(t, s) \mathrm{d} s, \quad w_{B}^{(k+1)}(t)=\int_{0}^{t} \Lambda_{B}^{(\nu)}\left(t, s, w_{B}^{(k)}\right) \mathrm{d} s+w_{B}^{(0)}(t) .
$$

We also set

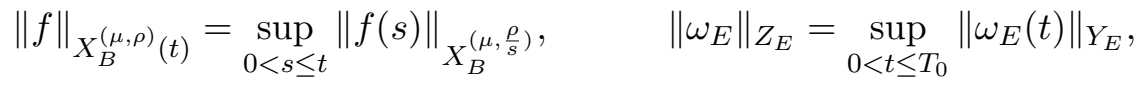

where $T_{0}>0$ is the number in $(2.3)$.

Lemma 4.1 Assume that $0<2^{-1}\left(\mu-\mu^{\prime}\right)<\mu^{\prime}<\mu<d_{E}, 0<\rho^{\prime}<\rho \leq 2^{-4}$, and $0<s<t<T_{0}$, where $T_{0} \in\left(0, d_{E}\right)$ is the number in (2.3). Then

$$
\begin{aligned}
& \left\|\Lambda_{B}^{(\nu)}(t, s, f)-\Lambda_{B}^{(\nu)}(t, s, h)\right\|_{X_{B}^{\left(\mu^{\prime}, \frac{\rho^{\prime}}{t}\right)}} \\
\lesssim & \left(\frac{1}{\mu-\mu^{\prime}}+\frac{s^{\frac{1}{2}}}{\mu^{\prime}(t-s)^{\frac{1}{2}}\left(\rho-\rho^{\prime}\right)^{\frac{1}{2}}}\right)\left(\left\|\omega_{E}\right\|_{Z_{E}}+\|f\|_{X_{B}^{(\mu, \rho)}(s)}+\|h\|_{X_{B}^{(\mu, \rho)}(s)}\right)\|f-h\|_{X_{B}^{(\mu, \rho)}(s)} .
\end{aligned}
$$

Proof. From $\cup_{0 \leq t \leq T_{0}} \operatorname{supp} \omega_{E}(t) \subset\left\{x \in \mathbb{R}_{+}^{2} \mid x_{2} \geq 32 d_{E}\right\}$ we have $\sup _{0 \leq t \leq T_{0}}\left\|\omega_{E}(t)\right\|_{X_{I I, 2}^{(\mu, N)} \cap Y_{E}} \leq 2\|\omega\|_{Z_{E}}$ for $0<\mu \leq 32 d_{E}$ and $N \geq 0$. Then, since $\Lambda_{B}^{(\nu)}(t, s, f)$ consists of the linear terms and the nonlinear terms which are bilinear forms, (4.11) follows from (3.36), (3.49), (3.51), and (3.54). The proof is complete.

Lemma 4.2 Assume that $0<\mu<d_{E}, 0<\rho \leq 2^{-4}$, and $0<s<t<T_{0}$, where $T_{0} \in\left(0, d_{E}\right)$ is the number in (2.3). Then

$$
\left\|F_{B}^{(\nu)}(t, s)\right\|_{X_{B}^{(\mu, \rho)}} \lesssim d_{E}^{-1}\left\|\omega_{E}\right\|_{Z_{E}}^{2}
$$

Proof. It is not difficult to see from $\cup_{0 \leq t \leq T_{0}}$ supp $\omega_{E}(t) \subset\left\{x \in \mathbb{R}_{+}^{2} \mid x_{2} \geq 32 d_{E}\right\}$ that the proof of (3.54) actually implies $\left\|\Upsilon_{2}^{(\nu)}\left(R_{\nu} \omega_{E}, R_{\nu} \omega_{E}\right)\right\|_{X_{B}^{\left(d_{E}, \rho / t\right)}} \lesssim d_{E}^{-1}\left\|\omega_{E}\right\|_{L^{\infty}\left(0, s ; X_{I I, 2}^{\left(2 d_{E}, 0\right)}\right)}^{2} \lesssim d_{E}^{-1}\left\|\omega_{E}\right\|_{Z_{E}}^{2}$. This estimate and (3.49) yield (4.12). The proof is complete.

For $\gamma_{0}>0$ we set

$$
\gamma_{k+1}=\gamma_{k}\left(1-(k+2)^{-2}\right), \quad \gamma=\lim _{k \rightarrow \infty} \gamma_{k}=\gamma_{0} \Pi_{k=0}^{\infty}\left(1-(k+2)^{-2}\right)>0 .
$$

A simple modification of the arguments in $[27,11]$ for the ACK theorem (see also $[26,31]$ ) leads to 
Lemma 4.3 Let $0<\nu<d_{E}$. Let $\mu_{0}=d_{E}, \rho_{0}=2^{-4}, \sigma_{0}=\left(\mu_{0}, \rho_{0}\right)$, and $0<\gamma_{0}<T_{0}$. Set

$$
\begin{aligned}
\lambda_{k} & =\sup _{\frac{1}{2} \leq \kappa<1} \sup _{0<t<\gamma_{k}(1-\kappa)}\left\|w_{B}^{(k)}\right\|_{X_{B}^{\kappa \sigma}(t)}, \\
\zeta_{k} & =\sup _{\frac{1}{2} \leq \kappa<1} \sup _{0<t<\gamma_{k}(1-\kappa)}\left\|w_{B}^{(k+1)}-w_{B}^{(k)}\right\|_{X_{B}^{\kappa \sigma}(t)}\left(\frac{\gamma_{k}(1-\kappa)}{t}-1\right) .
\end{aligned}
$$

If $\gamma_{0}$ is sufficiently small then it follows that $\lambda_{k} \leq 1$ and $\zeta_{k} \leq \delta^{k} \zeta_{0}$ for all $k \in \mathbb{N}$ and for some $\delta \in(0,1)$. Moreover, $\gamma_{0}$ is taken so that $\gamma_{0} \geq c_{0} d_{E}$, where $c_{0}>0$ depends only on $\|b\|_{Y_{E}}$.

Proof. Set $C_{E}=1+\left\|\omega_{E}\right\|_{Z_{E}}$. Note that $\gamma_{0}<T_{0} \leq d_{E}=\mu_{0}$. From Lemma 4.2 we first observe that for $0<\tilde{t} \leq t<\gamma_{0}(1-\kappa)$ and $1 / 2 \leq \kappa<1,\left\|w_{B}^{(0)}(\tilde{t})\right\|_{X_{B}^{\kappa\left(\mu_{0}, \rho_{0} / \tilde{t}\right)}} \lesssim \tilde{t} \mu_{0}^{-1}\left\|\omega_{E}\right\|_{Z_{E}}^{2} \leq \gamma_{0} \mu_{0}^{-1}\left\|\omega_{E}\right\|_{Z_{E}}^{2}$. Hence we have $\lambda_{0} \leq 1 / 4$ if $\gamma_{0} \approx c_{0} \mu_{0}$ with a sufficiently small $c_{0} \in(0,1)$ depending only on $\left\|\omega_{E}\right\|_{Z_{E}}$, and thus, on $\|b\|_{Y_{E}}$. Similarly, for $0<\tilde{t} \leq t<\gamma_{1}(1-\kappa)$ and $1 / 2 \leq \kappa<1$, Lemma 4.2 with $h=0$ implies

$$
\begin{aligned}
& \left\|w_{B}^{(1)}(\tilde{t})\right\|_{X_{B}^{\kappa\left(\mu_{0}, \frac{\rho_{0}}{t}\right)} \lesssim}^{\lesssim} \int_{0}^{\tilde{t}}\left\|\Lambda_{B}^{(\nu)}\left(\tilde{t}, s, w_{B}^{(0)}\right)\right\|_{X_{B}^{\kappa\left(\mu_{0}, \frac{\rho_{0}}{t}\right)}} \mathrm{d} s+\left\|w_{B}^{(0)}(\tilde{t})\right\|_{X_{B}^{\kappa\left(\mu_{0}, \frac{\rho_{0}}{t}\right)}} \\
\lesssim & \frac{1}{\mu_{0}} \int_{0}^{\tilde{t}}\left(\frac{1}{\kappa(s)-\kappa}+\frac{\tilde{t}^{\frac{1}{2}}}{(\tilde{t}-s)^{\frac{1}{2}}(\kappa(s)-\kappa)^{\frac{1}{2}}}\right)\left(\left\|\omega_{E}\right\|_{Z_{E}}+\left\|w_{B}^{(0)}\right\|_{X_{B}^{\kappa(s) \sigma_{0}}(s)}\right)\left\|w_{B}^{(0)}\right\|_{X_{B}^{\kappa(s) \sigma_{0}}(s)} \mathrm{d} s+\frac{1}{4},
\end{aligned}
$$

where $\kappa(s)=2^{-1}\left(1-s / \gamma_{0}+\kappa\right) \in(\kappa, 1)$. Since $\kappa(s)$ satisfies $s<\gamma_{0}(1-\kappa(s))$, we have

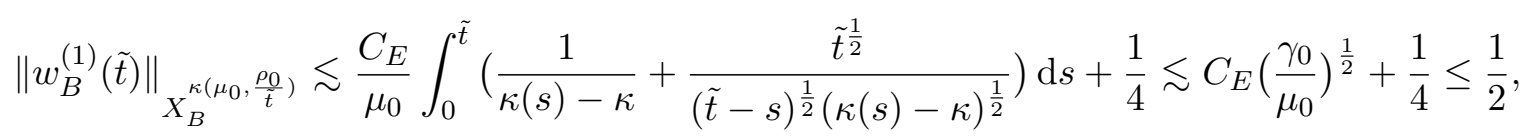

if $c_{0}$ chosen above is small enough (but depending only on $\|b\|_{Y_{E}}$ ). This shows $\lambda_{1} \leq 1 / 2$. Moreover, the calculation as above yields

$$
\begin{aligned}
& \left\|w_{B}^{(1)}(\tilde{t})-w_{B}^{(0)}(\tilde{t})\right\|_{X_{B}^{\kappa\left(\mu_{0}, \frac{\rho_{0}}{t}\right)}} \\
\lesssim & \frac{1}{\mu_{0}} \int_{0}^{\tilde{t}}\left(\frac{1}{\kappa(s)-\kappa}+\frac{\tilde{t}^{\frac{1}{2}}}{(\tilde{t}-s)^{\frac{1}{2}}(\kappa(s)-\kappa)^{\frac{1}{2}}}\right)\left(\left\|\omega_{E}\right\|_{Z_{E}}+\left\|w_{B}^{(0)}\right\|_{X_{B}^{\kappa(s) \sigma_{0}}(s)}\right)\left\|w_{B}^{(0)}\right\|_{X_{B}^{\kappa(s) \sigma_{0}}(s)} \mathrm{d} s,
\end{aligned}
$$

for $0<\tilde{t} \leq t<\gamma_{1}(1-\kappa), 1 / 2 \leq \kappa<1$, and $\kappa(s)=2^{-1}\left(1-s / \gamma_{1}+\kappa\right)$. Then it is easy to see

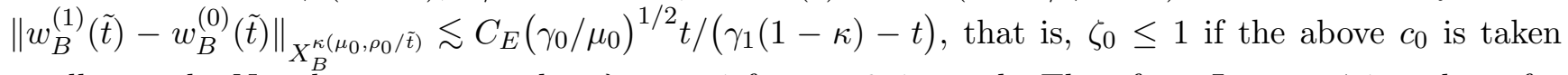
small enough. Now let us assume that $\lambda_{j+1} \leq 1$ for $j=0,1, \cdots, k$. Then from Lemma 4.1 we have for $0<\tilde{t} \leq t<\gamma_{k+1}(1-\kappa)$ and $1 / 2 \leq \kappa<1$,

$$
\begin{aligned}
& \left\|w_{B}^{(k+2)}(\tilde{t})-w_{B}^{(k+1)}(\tilde{t})\right\|_{X_{B}^{\kappa\left(\mu_{0}, \frac{\rho_{0}}{t}\right)}} \\
\lesssim & \frac{1}{\mu_{0}} \int_{0}^{\tilde{t}}\left(\frac{1}{\kappa(s)-\kappa}+\frac{\tilde{t}^{\frac{1}{2}}}{(\tilde{t}-s)^{\frac{1}{2}}(\kappa(s)-\kappa)^{\frac{1}{2}}}\right)\left(\left\|\omega_{E}\right\|_{Z_{E}}+\sum_{j=0,1}\left\|w_{B}^{(k+j)}\right\|_{X_{B}^{\kappa(s) \sigma_{0}}(s)}\right)\left\|w_{B}^{(k+1)}-w_{B}^{(k)}\right\|_{X_{B}^{\kappa(s) \sigma_{0}}(s)} \mathrm{d} s \\
\lesssim & \frac{C_{E}}{\mu_{0}} \int_{0}^{\tilde{t}}\left(\frac{1}{\kappa(s)-\kappa}+\frac{\tilde{t}^{\frac{1}{2}}}{(\tilde{t}-s)^{\frac{1}{2}}(\kappa(s)-\kappa)^{\frac{1}{2}}}\right)\left\|w_{B}^{(k+1)}-w_{B}^{(k)}\right\|_{X_{B}^{\kappa(s) \sigma_{0}}(s)} \mathrm{d} s,
\end{aligned}
$$


where $\kappa(s)=2^{-1}\left(1-s / \gamma_{k+1}+\kappa\right)$. Since $s<\gamma_{k}(1-\kappa(s))$ and $\kappa(s) \in(\kappa, 1)$ for $0<s<\tilde{t}$, we get

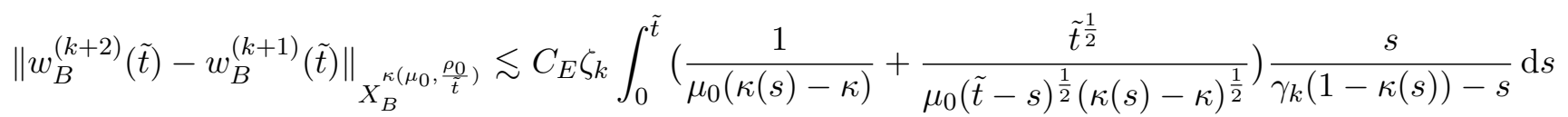

$$
\begin{aligned}
& \lesssim C_{E} \zeta_{k} \tilde{t} \int_{0}^{\tilde{t}}\left(\frac{\gamma_{k+1}}{\mu_{0}\left(\gamma_{k+1}(1-\kappa)-s\right)^{2}}+\frac{\gamma_{k+1}^{\frac{1}{2}}}{\mu_{0}^{\frac{1}{2}}(\tilde{t}-s)^{\frac{1}{2}}\left(\gamma_{k+1}(1-\kappa)-s\right)^{\frac{3}{2}}}\right) \mathrm{d} s \\
& \lesssim C_{E} \zeta_{k}\left(\frac{\gamma_{0}}{\mu_{0}}\right)^{\frac{1}{2}} \frac{\tilde{t}}{\gamma_{k+1}(1-\kappa)-\tilde{t}},
\end{aligned}
$$

which gives $\zeta_{k+1} \lesssim C_{E}\left(\gamma_{0} / \mu_{0}\right)^{1 / 2} \zeta_{k}$, i.e., $\zeta_{k+1} \leq \delta \zeta_{k} \leq \delta^{k+1} \zeta_{0}$ for $\delta \in(0,1)$ if the above $c_{0}$ is sufficiently small depending only on $\|b\|_{Y_{E}}$. Using this estimate and the definitions of $\lambda_{k}, \zeta_{k}$, and $\gamma_{k}$, we also have

$$
\lambda_{k+2} \leq \frac{\gamma_{k+2}}{\gamma_{k+1}-\gamma_{k+2}} \zeta_{k+1}+\lambda_{k+1} \lesssim(k+3)^{2} \delta^{k+1} \zeta_{0}+\lambda_{k+1} \leq \zeta_{0} \sum_{j=0}^{k}(j+3)^{3} \delta^{j+1}+\lambda_{1} .
$$

Since $\zeta_{0} \leq 1$ and $\lambda_{1} \leq 1 / 2$, if $c_{0}$ is taken small enough depending only on $\|b\|_{Y_{E}}$ then we have $\lambda_{k+2} \leq 1$. Hence the assertion of the lemma follows by the induction on $k$. The proof is complete.

Lemma 4.3 implies the following existence theorem for (4.1).

Theorem 4.4 There is $T_{B} \in\left(0, T_{0}\right)$ such that (4.1) admits the unique solution $w_{B}$ which belongs to the space $C\left(\left[0, T_{B}\right) ; X_{B_{\nu}}^{\left(\mu_{B}, \rho_{B} / T_{B}\right)}\right)$ with $\mu_{B}=d_{E} / 2, \rho_{B}=2^{-5}$, and satisfies $\sup _{0<t<T_{B}}\left\|w_{B}(t)\right\|_{X_{B}^{\left(\mu_{B}, \rho_{B} / t\right)}} \leq 1$. Moreover, $T_{B}$ is taken so that $T_{B} \gtrsim c_{0} d_{E}$, where $c_{0}>0$ is the number in Lemma 4.3.

Proof. Lemma 4.3 shows that $\left\{w_{B}^{(k)}\right\}_{k=0}^{\infty}$ is a Cauchy sequence in the Banach space endowed with the norm $\|F\|=\sup _{1 / 2 \leq \kappa<1} \sup _{0<t<\gamma(1-\kappa)}\|F\|_{X_{B}^{\kappa \sigma_{0}(t)}}\left(\frac{\gamma(1-\kappa)}{t}-1\right)$, where $\gamma>0$ is defined by (4.13) with $\gamma_{0}>0$ in Lemma 4.3. Let $w_{B}$ be the limit of $\left\{w_{B}^{(k)}\right\}_{k=0}^{\infty}$. Then Lemma 4.3 implies $\sup _{1 / 2 \leq \kappa<1} \sup _{0<t<\gamma(1-\kappa)}\left\|w_{B}\right\|_{X_{B}^{\kappa \sigma_{0}(t)}} \leq$ 1. By Lemmas $4.1,4.2$ we see that $w_{B}$ is the solution of $(4.1)$ belonging to $C\left(\left[0, T_{1}\right) ; X_{B}^{\left(\mu, \rho / T_{B}\right)}\right)$ with $\mu=d_{E} / 2$ and $\rho=2^{-5}$, where $T_{B}=\gamma_{0} / 2$. The proof is complete.

\subsection{Solutions of $\left(\mathrm{V}_{\mathrm{I}_{\nu}}\right)$}

In this section we construct the remainder part $\omega_{I}=\omega-\omega_{E}-R_{1 / \nu} w_{B}$, where $w_{B}$ is the boundary layer function in Theorem 4.4. Our aim is to solve the integral equation

$$
w_{I}(t)=\int_{0}^{t} \Lambda_{I}^{(\nu)}\left(t, s, w_{I}\right) \mathrm{d} s+\int_{0}^{t} F_{I}^{(\nu)}(t, s) \mathrm{d} s
$$

where $w_{I}=\left(w_{I B}, w_{I I}\right), \Lambda_{I}^{(\nu)}\left(t, s, w_{I}\right)=\left(\Lambda_{I B}^{(\nu)}\left(t, s, w_{I}\right), \Lambda_{I I}^{(\nu)}\left(t, s, w_{I}\right)\right)$, and $F_{I}^{(\nu)}=\left(F_{I B}^{(\nu)}, F_{I I}^{(\nu)}\right)$. This solution is shown to give $\omega_{I}$ of the form $\omega_{I}=R_{1 / \nu} w_{I B}+w_{I I}$. To apply the ACK theorem we consider the iteration sequence $\left\{w_{I}^{(k)}\right\}_{k=0}^{\infty}$,

$$
w_{I}^{(0)}(t)=\int_{0}^{t} F_{I}^{(\nu)}(t, s) \mathrm{d} s, \quad w_{I}^{(k+1)}(t)=\int_{0}^{t} \Lambda_{I}^{(\nu)}\left(t, s, w_{I}^{(k)}\right) \mathrm{d} s+w_{I}^{(0)}(t) .
$$


Let $T_{B}>0$ be the number in Theorem 4.4. We set

$$
\begin{array}{ll}
\left\|w_{B}\right\|_{Z_{B}}=\sup _{0<t<T_{B}}\left\|w_{B}(t)\right\|_{\left.X_{B}^{\left(\mu_{B},\right.}, \frac{\rho_{B}}{t}\right)} & \mu_{B}=\frac{d_{E}}{2}, \quad \rho_{B}=2^{-5}, \\
\left\|f_{I}\right\|_{X_{I, j}^{(\mu, \rho, \theta)}}=\left\|f_{I B}\right\|_{X_{I B, j}^{(\mu, \rho)}}+\left\|f_{I I}\right\|_{X_{I I, j}^{(\mu, \theta)}} & f_{I}=\left(f_{I B}, f_{I I}\right), \\
\left\|f_{I}\right\|_{X_{I, j}^{\sigma}(t)}=\sup _{0<s \leq t}\left\|f_{I}(s)\right\|_{X_{I, j}^{\left(\mu, \rho, \frac{\theta}{s}\right)}} & f_{I}(s)=\left(f_{I B}(s), f_{I I}(s)\right), \quad \sigma=(\mu, \rho, \theta) .
\end{array}
$$

Lemma 4.5 Assume that $0<2^{-1}\left(\mu-\mu^{\prime}\right)<\mu^{\prime}<\mu<d_{E} / 2,0<\rho^{\prime}<\rho \leq 2^{-5}, 0<\theta \leq 2^{-8}$, and $0<s<t<T_{B}$, where $T_{B}$ is the number in Theorem 4.4. Set $\sigma=(\mu, \rho, \theta)$ and $C_{E}=1+\left\|\omega_{E}\right\|_{Z_{E}}$. Let $j=0,1$. Then

$$
\begin{aligned}
\| \Lambda_{I B}^{(\nu)}\left(t, s, f_{I}\right)- & \Lambda_{I B}^{(\nu)}\left(t, s, h_{I}\right)\left\|_{\left.X_{I B, j}^{\left(\mu^{\prime}, \rho^{\prime}\right.}\right)} \lesssim C_{E}\left(\frac{1}{\mu-\mu^{\prime}}+\frac{s^{\frac{1}{2}}}{\mu^{\prime}(t-s)^{\frac{1}{2}}\left(\rho-\rho^{\prime}\right)^{\frac{1}{2}}}\right)\right\| f_{I}-h_{I} \|_{X_{I, j}^{\sigma}(s)} \\
& +\left(\frac{1}{\nu^{\frac{1}{2}}(t-s)^{\frac{1}{2}}}+\frac{\nu^{\frac{1}{4}} s^{\frac{1}{2}}}{(t-s)^{\frac{1}{4}}\left(\rho-\rho^{\prime}\right)^{\frac{1}{2}}}\right)\left(\left\|f_{I}\right\|_{X_{I, 1}^{\sigma}(s)}+\left\|h_{I}\right\|_{X_{I, 1}^{\sigma}(s)}\right)\left\|f_{I}-h_{I}\right\|_{X_{I, j}^{\sigma}(s)} .
\end{aligned}
$$

Proof. We note that $\Lambda_{I B}^{(\nu)}\left(t, s, f_{I}\right)$ consists of the linear terms and nonlinear terms which are bilinear forms. Hence (4.19) directly follows from Lemmas $3.4-3.7$ and $\left\|w_{B}\right\|_{Z_{B}} \leq 1$. The details are omitted. The proof is complete.

Lemma 4.6 Assume that $0<\mu<d_{E} / 2,0<\rho \leq 2^{-5}$, and $0<s<t<T_{B}$, where $T_{B} \in\left(0, d_{E}\right)$ is the number in Theorem 4.4. Let $0<\nu<d_{E}^{2}$. Then

$$
\left\|F_{I B}^{(\nu)}(t, s)\right\|_{X_{I B, 1}^{\left(\mu, \frac{\rho}{t}\right)}} \lesssim \frac{\nu^{\frac{1}{2}}}{d_{E}}\left\|\omega_{E}\right\|_{Z_{E}}
$$

Proof. It is not difficult to see that the terms $\left\|R_{\nu} e^{\nu(t-s) \Delta_{N}}\left(J_{1}\left(\Delta \omega_{E}\right) \mathcal{H}_{\left\{x_{2}=0\right\}}^{1}\right)\right\|_{X_{I B, 1}^{(\mu, \rho / t)}}$ and $\nu \| R_{\nu} \int_{0}^{s} \Xi G(\nu(s-$ $\tau)) \star\left(\Delta \omega_{E}-J_{1}\left(\Delta \omega_{E}\right) \mathcal{H}_{\left\{x_{2}=0\right\}}^{1}\right) \mathrm{d} \tau \|_{X_{I B, 1}^{(\mu, \rho / t)}}$ are bounded from above by $C\left\|\omega_{E}\right\|_{Z_{E}}$ for some constant $C>0$. The details are omitted. In the sequel we give the estimate only for $\Phi_{B, 2}^{(\nu)}\left[w_{B}, R_{\nu} \omega_{E}\right]$ in the definition of $F_{I B}^{(\nu)}$, for the other terms are estimated in the similar manner. Since supp $R_{\nu} \omega_{E}(t) \subset\left\{X \in \mathbb{R}_{+}^{2} \mid X_{2} \geq\right.$ $\left.32 \nu^{-1 / 2} d_{E}\right\}$ we observe from (3.34) and from the calculations as in (3.26) and (3.28),

$$
\begin{aligned}
& \left|\mathcal{F}\left(N^{(\nu)}\left(w_{B}, R_{\nu} \omega_{E}\right)\right)\left(\xi_{1}\right)\right| \leq \sum_{j=1}^{2} \int_{0}^{\infty} e^{-\nu^{\frac{1}{2}}\left|\xi_{1}\right| Y_{2}}\left|\xi_{1}\right| \nu^{\frac{j-1}{2}}\left|\mathcal{F}\left(D_{j}^{(\nu)}\left(w_{B}, R_{\nu} \omega_{E}\right)\right)\right|\left(\xi_{1}, Y_{2}\right) \mathrm{d} Y_{2} \\
\lesssim & \int_{\frac{32 d_{E}}{\nu^{1 / 2}}}^{\infty}\left|\xi_{1}\right| e^{-\nu^{\frac{1}{2}}\left|\xi_{1}\right| Y_{2}}\left\|\left(\nu^{\frac{1}{2}}\left|\eta_{1}\right|\left\|\chi_{\left\{Z_{2} \leq Y_{2}\right\}} \hat{w_{B}}\left(\eta_{1}\right)\right\|_{L_{Z_{2}}^{1}}+\left\|\chi_{\left\{Z_{2} \geq Y_{2}\right\}} \hat{w_{B}}\left(\eta_{1}\right)\right\|_{L_{Z_{2}}^{1}}\right) R_{\nu} \hat{\omega_{E}}\left(\xi_{1}-\eta_{1}, Y_{2}\right)\right\|_{L_{\eta_{1}}^{1}} \mathrm{~d} Y_{2} \\
\lesssim & d_{E}^{-1} e^{-2 d_{E}\left|\xi_{1}\right|}\left\|\left(\nu^{\frac{1}{2}}\left|\eta_{1}\right|\left\|\hat{w}_{B}\left(\eta_{1}\right)\right\|_{L_{X_{2}}^{1}}+e^{-\frac{1}{\nu s} d_{E}^{2}}\left\|\varphi_{B}^{\left(0, \frac{1}{2^{5} s}\right)} \hat{w}_{B}\left(\eta_{1}\right)\right\|_{L_{X_{2}}^{1}}\right)\left(1+\left|\xi_{1}-\eta_{1}\right|\right)^{-4}\right\|_{L_{\eta_{1}}^{1}}\left\|\omega_{E}\right\|_{W^{4,1}},
\end{aligned}
$$

which implies

$$
\left\|e^{d_{E}\left|\xi_{1}\right|}\left\langle\xi_{1}\right\rangle \mathcal{F}\left(N^{(\nu)}\left(w_{B}(s), R_{\nu} \omega_{E}(s)\right)\right)\right\|_{L_{\xi_{1}}^{2}} \lesssim \frac{1}{d_{E}}\left(\nu^{\frac{1}{2}}+\frac{\nu s}{d_{E}^{2}}\right)\left\|\omega_{E}\right\| Z_{Z_{E}}\left\|w_{B}\right\|_{Z_{B}} \lesssim \frac{\nu^{\frac{1}{2}}}{d_{E}}\left\|\omega_{E}\right\|_{Z_{E}}\left\|w_{B}\right\|_{Z_{B}} .
$$


Here we have used $0<s<t<T_{B}<d_{E}$ and $\nu<d_{E}^{2}$. Hence we have from (3.14),

$$
\left\|\Phi_{B, 2}^{(\nu)}\left[w_{B}(s), R_{\nu} \omega_{E}(s)\right](t-s)\right\|_{X_{I B, 1}^{(\mu, \rho)},} \lesssim\left\|e^{d_{E}\left|\xi_{1}\right|}\left\langle\xi_{1}\right\rangle \mathcal{F}\left(N^{(\nu)}\left(w_{B}(s), R_{\nu} \omega_{E}(s)\right)\right)\right\|_{L_{\xi_{1}}^{2}} \lesssim \frac{\nu^{\frac{1}{2}}}{d_{E}}\left\|\omega_{E}\right\|_{Z_{E}},
$$

by $\left\|w_{B}\right\|_{Z_{B}} \leq 1$. The proof is complete.

Lemma 4.7 Assume that $0<2^{-1}\left(\mu-\mu^{\prime}\right)<\mu^{\prime}<\mu<d_{E} / 2,2^{-7} \leq \rho^{\prime}<\rho \leq 2^{-5}, 0<\theta \leq 2^{-8}$, and $0<s<t<\min \left\{T_{0}^{\prime}, T_{B}\right\}$, where $T_{0}^{\prime}, T_{B}$ are the numbers in Proposition 3.13, Theorem 4.4, respectively. Set $\sigma=(\mu, \rho, \theta)$ and $C_{E}=1+\left\|\omega_{E}\right\|_{Z_{E}}$. Let $f_{I}=\left(f_{I B}, f_{I I}\right)$ satisfy (3.85) and $0<\nu<d_{E}^{5}$. Then

$$
\begin{gathered}
\left\|\Lambda_{I I}^{(\nu)}\left(t, s, f_{I}\right)\right\|_{X_{I I, 1}^{\left(\mu^{\prime}, \theta^{\prime}\right.} t} \lesssim C_{E}^{2}\left(1+\frac{s^{\frac{1}{2}}}{d_{E}(t-s)^{\frac{1}{2}}\left(\theta-\theta^{\prime}\right)^{\frac{1}{2}}}\right)\left\|f_{I}\right\|_{X_{I, 1}^{\sigma}(s)}+\frac{1}{\nu^{\frac{1}{2}}(t-s)^{\frac{1}{2}}\left(\theta-\theta^{\prime}\right)^{\frac{1}{4}}}\left\|f_{I}\right\|_{X_{I, 1}^{\sigma}(s)}^{2} \\
+\frac{C_{E}^{2}}{d_{E}}\left(1+\frac{s^{\frac{1}{2}}}{(t-s)^{\frac{1}{2}}}\right) \nu^{\frac{1}{2}} .
\end{gathered}
$$

Moreover, if $h_{I}=\left(h_{I B}, h_{I I}\right)$ satisfies (3.85) in addition, then

$$
\left\|\Lambda_{I I}^{(\nu)}\left(t, s, f_{I}\right)-\Lambda_{I I}^{(\nu)}\left(t, s, h_{I}\right)\right\|_{X_{I I, 0}^{\left(\mu^{\prime}, \theta^{\prime}\right.} t} \lesssim\left(\frac{C_{E} s^{\frac{1}{2}}}{d_{E}\left(\theta-\theta^{\prime}\right)^{\frac{1}{2}}}+\frac{\left\|f_{I}\right\|_{X_{I, 1}^{\sigma}(s)}+\left\|h_{I}\right\|_{X_{I, 1}^{\sigma}(s)}}{\nu^{\frac{1}{2}}\left(\theta-\theta^{\prime}\right)^{\frac{1}{4}}}+C_{E}^{2}\right) \frac{\left\|f_{I}-h_{I}\right\|_{X_{I, 0}^{\sigma}(s)}}{(t-s)^{\frac{1}{2}}} .
$$

Proof. To make the notation short the terms like $\Phi_{I}^{(\nu)}\left[R_{1 / \nu} f_{I B}+f_{I I}, \omega_{E}\right](t-s)$ will be denoted by $\Phi_{I}^{(\nu)}\left[R_{1 / \nu} f_{I B}+f_{I I}, \omega_{E}\right]$. From supp $\omega_{E}(t) \subset\left\{x \in \mathbb{R}_{+}^{2} \mid x_{2} \geq 32 d_{E}\right\}$ we apply (3.66) to get

$$
\begin{aligned}
& \left\|\Phi_{I}^{(\nu)}\left[R_{\frac{1}{\nu}} f_{I B}+f_{I I}, \omega_{E}\right]\right\|_{X_{I I, 1}^{\left(\mu^{\prime}, \frac{\theta^{\prime}}{t}\right)}}=\left\|\Phi_{I, 3}^{(\nu)}\left[R_{\frac{1}{\nu}} f_{I B}+f_{I I}, \omega_{E}\right]\right\|_{X_{I I, 1}^{\left(\mu^{\prime}, \frac{\theta^{\prime}}{t}\right)}} \\
\lesssim & \left(\left(\frac{\nu^{\frac{1}{2}} s^{\frac{1}{2}}}{d_{E}^{\frac{3}{2}} \rho^{\frac{1}{2}}}+\frac{e^{-\frac{\rho}{\nu s} d_{E}^{2}}}{d_{E}}\right)\left\|f_{I B}(s)\right\|_{X_{I B, 1}^{\left(0, \frac{\rho}{s}\right)}}+\left\|f_{I I}(s)\right\|_{X_{I I, 1}^{(0,0)}}\right)\left\|\omega_{E}\right\|_{Z_{E}} \lesssim C_{E}\left\|f_{I}\right\|_{X_{I, 1}^{\sigma}(s)}
\end{aligned}
$$

for $0<\nu<d_{E}^{2}, 0<s<d_{E}$, and $2^{-7} \leq \rho^{\prime}<\rho<2^{-5}$. Similarly, we have

$$
\left\|\Phi_{I}^{(\nu)}\left[R_{\frac{1}{\nu}} f_{I B}+f_{I I}-R_{\frac{1}{\nu}} h_{I B}-h_{I I}, \omega_{E}\right]\right\|_{X_{I I, 0}^{\left(\mu^{\prime}, \frac{\theta^{\prime}}{t}\right)}} \lesssim C_{E}\left\|f_{I}-h_{I}\right\|_{X_{I, 0}^{\sigma}(s)} .
$$

Next we observe from Lemma 3.8 that

$$
\begin{aligned}
\left\|\Phi_{I, 1}^{(\nu)}\left[\omega_{E}+R_{\frac{1}{\nu}} w_{B}, f_{I I}\right]\right\|_{X_{I I, 1}^{\left(\mu^{\prime}, \frac{\theta^{\prime}}{t}\right)}} \lesssim \frac{s^{\frac{1}{2}}\left(\left\|\omega_{E}\right\|_{Z_{E}}+\left\|w_{B}\right\|_{Z_{B}}\right)}{d_{E}(t-s)^{\frac{1}{2}}\left(\theta-\theta^{\prime}\right)^{\frac{1}{2}}}\left\|f_{I}\right\|_{X_{I, 1}^{\sigma}(s)} \leq \frac{C_{E} s^{\frac{1}{2}}}{d_{E}(t-s)^{\frac{1}{2}}\left(\theta-\theta^{\prime}\right)^{\frac{1}{2}}}\left\|f_{I}\right\|_{X_{I, 1}^{\sigma}(s)}, \\
\left\|\Phi_{I, 1}^{(\nu)}\left[R_{\frac{1}{\nu}} f_{I B}+f_{I I}, f_{I I}\right]\right\|_{X_{I I, 1}^{\left(\mu^{\prime}, \frac{\theta^{\prime}}{t}\right)}} \lesssim \frac{s^{\frac{1}{4}}\left(\left\|f_{I B}(s)\right\|_{X_{I B, 1}^{(\mu, 0)}+d_{E}^{-\frac{3}{2}}}\left\|f_{I I}(s)\right\|_{\left.X_{I I, 1}^{(\mu, 0)}\right)}\left\|f_{I I}(s)\right\|_{X_{I I, 1}^{\left(\mu, \frac{\theta}{s}\right)}}\right.}{d_{E}^{\frac{1}{2}} \nu^{\frac{1}{4}}(t-s)^{\frac{1}{2}}\left(\theta-\theta^{\prime}\right)^{\frac{1}{4}}} \\
\lesssim \frac{s^{\frac{1}{4}}}{d_{E}^{2} \nu^{\frac{1}{4}}(t-s)^{\frac{1}{2}}\left(\theta-\theta^{\prime}\right)^{\frac{1}{4}}}\left\|f_{I}\right\|_{X_{I, 1}^{\sigma}(s)}^{2} .
\end{aligned}
$$


Similarly, Lemma 3.8 implies

$$
\begin{aligned}
& \left\|\Phi_{I, 1}^{(\nu)}\left[\omega_{E}+R_{\frac{1}{\nu}} w_{B}, f_{I I}-h_{I I}\right]\right\|_{X_{I I, 0}^{\left(\mu^{\prime}, \frac{\theta^{\prime}}{t}\right)}}+\left\|\Phi_{I, 1}^{(\nu)}\left[R_{\frac{1}{\nu}} f_{I B}+f_{I I}, f_{I I}\right]-\Phi_{I, 1}^{(\nu)}\left[R_{\frac{1}{\nu}} h_{I B}+h_{I I}, h_{I I}\right]\right\|_{X_{I I, 0}^{\left(\mu^{\prime}, \frac{\theta^{\prime}}{t}\right)}} \\
\lesssim & \frac{C_{E} s^{\frac{1}{2}}}{d_{E}(t-s)^{\frac{1}{2}}\left(\theta-\theta^{\prime}\right)^{\frac{1}{2}}}\left\|f_{I}-h_{I}\right\|_{X_{I, 0}^{\sigma}(s)}+\frac{s^{\frac{1}{4}}\left(\left\|f_{I}\right\|_{X_{I, 1}^{\sigma}(s)}+\left\|h_{I}\right\|_{X_{I, 1}^{\sigma}(s)}\right)}{d_{E}^{\frac{3}{2}} \nu^{\frac{1}{4}}(t-s)^{\frac{1}{2}}\left(\theta-\theta^{\prime}\right)^{\frac{1}{4}}}\left\|f_{I}-h_{I}\right\|_{X_{I, 0}^{\sigma}(s)} .
\end{aligned}
$$

As for the term $\Phi_{I, 2}^{(\nu)}\left[\omega_{E}+R_{1 / \nu} w_{B}, H^{(\nu)}\left[f_{I}\right]\right]$, we have from (3.64) and Proposition 3.11,

$$
\begin{aligned}
&\left\|\Phi_{I, 2}^{(\nu)}\left[\omega_{E}+R_{\frac{1}{\nu}} w_{B}, H^{(\nu)}\left[f_{I}\right]\right]\right\|_{X_{I I, 1}^{\left(\mu^{\prime}, \frac{\theta^{\prime}}{t}\right)}} \lesssim \frac{\left\|\omega_{E}\right\|_{Z_{E}}+\left\|w_{B}\right\|_{Z_{B}}}{d_{E}^{\frac{3}{2}} \nu^{\frac{1}{2}}(t-s)^{\frac{1}{2}}} e^{\frac{1}{64 \nu s} d_{E}^{2}}\left\|\chi_{4 d_{E}}^{c} \chi_{8 d_{E}} H^{(\nu)}\left[f_{I}\right](s)\right\|_{W^{1,2} \cap L^{\frac{4}{3}}} \\
& \lesssim \frac{C_{E}}{d_{E}^{\frac{3}{2}} \nu^{\frac{1}{2}}(t-s)^{\frac{1}{2}}} e^{-\frac{1}{32 \nu s} d_{E}^{2}}\left\|\Omega\left[f_{I}\right]\right\|_{L^{1}\left(0, s ; L^{1}\right)},
\end{aligned}
$$

where

$$
\Omega\left[f_{I}\right](\tau)=-B\left(R_{\frac{1}{\nu}} w_{B}(\tau)+R_{\frac{1}{\nu}} f_{I B}(\tau)+f_{I I}(\tau), \omega_{E}(\tau)\right)+\nu \Delta \omega_{E}(\tau) .
$$

Then Lemma 2.6 implies

$$
\left\|\Omega\left[f_{I}\right](\tau)\right\|_{L^{1}} \lesssim\left(\frac{\nu^{\frac{1}{2}} \tau^{\frac{1}{2}}}{d_{E}}\left(\left\|w_{B}(\tau)\right\|_{X_{I B, 0}^{\left(0, \frac{1}{2^{7} \tau}\right)}}+\left\|f_{I B}(\tau)\right\|_{X_{I B, 0}^{\left.\left(0, \frac{1}{2}\right)^{7}\right)}}\right)+\left\|f_{I I}(\tau)\right\|_{X_{I I, 0}^{(0,0)}}\right)\left\|\omega_{E}\right\|_{Z_{E}}+\nu\left\|\omega_{E}\right\|_{Z_{E}},
$$

and thus, since $0<s<d_{E}$ we have from $\left\|w_{B}\right\|_{Z_{B}} \leq 1$,

$$
\left\|\Omega\left[f_{I}\right]\right\|_{L^{1}\left(0, s ; L^{1}\right)} \lesssim C_{E}\left(s\left\|f_{I}\right\|_{X_{I, 0}^{\left(0, \frac{1}{2^{7}}, 0\right)}(s)}+\nu^{\frac{1}{2}}\right) .
$$

Hence (4.28) and (4.30) yield

$$
\left\|\Phi_{I, 2}^{(\nu)}\left[\omega_{E}+R_{\frac{1}{\nu}} w_{B}, H^{(\nu)}\left[f_{I}\right]\right]\right\|_{X_{I I, 1}^{\left(\mu^{\prime}, \frac{\theta^{\prime}}{t}\right)} \lesssim} \frac{C_{E}^{2} s^{\frac{1}{2}}}{(t-s)^{\frac{1}{2}}}\left(s\left\|f_{I}\right\|_{X_{I, 0}^{\left(0, \frac{1}{2^{7}}, 0\right)}(s)}+\nu^{\frac{1}{2}}\right)
$$

for $0<\nu<d_{E}^{5}$. Similarly, we have

$$
\left\|\Phi_{I, 2}^{(\nu)}\left[R_{\frac{1}{\nu}} f_{I B}+f_{I I}, H^{(\nu)}\left[f_{I}\right]\right]\right\|_{X_{I I, 1}^{\left(\mu^{\prime}, \theta^{\prime}\right)}} \lesssim \frac{C_{E} s^{\frac{1}{2}}}{(t-s)^{\frac{1}{2}}}\left\|f_{I}\right\|_{X_{I, 0}^{\left(0, \frac{1}{2^{7}}, 0\right)}(s)}\left(s\left\|_{I}\right\|_{X_{I, 0}^{\left(0, \frac{1}{2^{7}}, 0\right)}(s)}+\nu^{\frac{1}{2}}\right) .
$$

On the other hand, as in the calculation of (4.28), we have from (3.64) and Proposition 3.14,

$$
\begin{aligned}
& \left\|\Phi_{I, 2}^{(\nu)}\left[\omega_{E}+R_{\frac{1}{\nu}} w_{B}, H^{(\nu)}\left[f_{I}\right]-H^{(\nu)}\left[h_{I}\right]\right]\right\|_{X_{I I, 0}^{\left(\mu^{\prime}, \frac{\theta^{\prime}}{t}\right)}} \\
\lesssim & \frac{\left\|\omega_{E}\right\|_{Z_{E}}+\left\|w_{B}\right\|_{Z_{B}} e^{\frac{1}{64 \nu s} d_{E}^{2}}\left\|\chi_{4 d_{E}}^{c} \chi_{8 d_{E}}\left(H^{(\nu)}\left[f_{I}\right](s)-H^{(\nu)}\left[h_{I}\right](s)\right)\right\|_{L^{2} \cap L^{\frac{1}{3}}}}{d_{E} \nu^{\frac{1}{2}}(t-s)^{\frac{1}{2}}} \\
\lesssim & \frac{C_{E} e^{-\frac{1}{32 \nu s} d_{E}^{2}}}{d_{E} \nu^{\frac{1}{2}}(t-s)^{\frac{1}{2}}}\left(\left(\left\|\nabla \Omega\left[f_{I}\right]\right\|_{L^{1}\left(0, s ; L^{\frac{4}{3}}\right)}+\left\|\Omega\left[f_{I}\right]\right\|_{L^{1}\left(0, s ; L^{1}\right)}\right)\left\|f_{I}-h_{I}\right\|_{X_{I, 0}^{(0,0,0)}(s)}+\left\|\Omega\left[f_{I}\right]-\Omega\left[h_{I}\right]\right\|_{L^{1}\left(0, s ; L^{1}\right)}\right) .
\end{aligned}
$$


From Lemma 2.6 it is not difficult to see that for $0<\nu<d_{E}^{5}$ and $0<\tau<s<d_{E}$,

$$
\begin{aligned}
\left\|\nabla \Omega\left[f_{I}\right](\tau)\right\|_{W^{1,2} \cap L^{\frac{4}{3}}} & \lesssim\left(\left(\frac{\nu \tau}{d_{E}^{5}}\right)^{\frac{1}{2}}\left(\left\|w_{B}(\tau)\right\|_{X_{I B, 1}^{\left(0, \frac{1}{2^{7} \tau}\right)}}+\left\|f_{I B}(\tau)\right\|_{\left.X_{I B, 1}^{\left(0, \frac{1}{2^{7}}\right)}\right)}\right)+\left\|f_{I I}(\tau)\right\|_{X_{I I, 1}^{(0,0)}}\right)\left\|\omega_{E}\right\|_{Z_{E}}+\nu\left\|\omega_{E}\right\|_{Z_{E}} \\
& \lesssim C_{E}\left\|f_{I}\right\|_{X_{I, 1}^{\left(0, \frac{1}{2^{7}}, 0\right)}(s)}+C_{E} \nu^{\frac{1}{2}} d_{E}^{-2}
\end{aligned}
$$

which gives

$$
\left\|\nabla \Omega\left[f_{I}\right]\right\|_{L^{1}\left(0, s ; W^{1,2} \cap L^{\frac{4}{3}}\right)} \lesssim C_{E}\left(s\left\|f_{I}\right\|_{X_{I, 1}^{\left(0, \frac{1}{2}^{7}, 0\right)}(s)}+\nu^{\frac{1}{2}} d_{E}^{-1}\right) .
$$

Lemma 2.6 also implies

$$
\left\|\Omega\left[f_{I}\right]-\Omega\left[h_{I}\right]\right\|_{L^{1}\left(0, s ; L^{p}\right)}+\left\|\nabla \Omega\left[f_{I}\right]-\nabla \Omega\left[h_{I}\right]\right\|_{L^{1}\left(0, s ; L^{2} \cap L^{\frac{4}{3}}\right)} \lesssim C_{E} s\left\|f_{I}-h_{I}\right\|_{X_{I, 0}^{\left(0, \frac{1}{2^{7}}, 0\right)}(s)}
$$

for $1 \leq p \leq 4,0<\nu<d_{E}^{5}$, and $0<s<d_{E}$. Hence the estimates (4.30), (4.33), (4.34), and (4.35) show

$$
\left\|\Phi_{I, 2}^{(\nu)}\left[\omega_{E}+R_{\frac{1}{\nu}} w_{B}, H^{(\nu)}\left[f_{I}\right]-H^{(\nu)}\left[h_{I}\right]\right]\right\|_{X_{I I, 0}^{\left(\mu^{\prime}, \frac{\theta^{\prime}}{t}\right)}} \lesssim \frac{C_{E}^{2}}{(t-s)^{\frac{1}{2}}}\left(1+\left\|f_{I}\right\|_{X_{I, 1}^{\left(0, \frac{1}{2^{7}}, 0\right)}(s)}\right)\left\|f_{I}-h_{I}\right\|_{X_{I, 0}^{\sigma}(s)} .
$$

Similarly, we have

$$
\begin{aligned}
& \left\|\Phi_{I, 2}^{(\nu)}\left[R_{\frac{1}{\nu}} f_{I B}+f_{I I}, H^{(\nu)}\left[f_{I}\right]\right]-\Phi_{I, 2}^{(\nu)}\left[R_{\frac{1}{\nu}} h_{I B}+h_{I I}, H^{(\nu)}\left[h_{I}\right]\right]\right\|_{X_{I I, 0}^{\left(\mu^{\prime}, \frac{\theta^{\prime}}{t}\right)}} \\
& \leq\left\|\Phi_{I, 2}^{(\nu)}\left[R_{\frac{1}{\nu}} f_{I B}-R_{\frac{1}{\nu}} h_{I B}+f_{I I}-h_{I I}, H^{(\nu)}\left[f_{I}\right]\right]\right\|_{X_{I I, 0}^{\left(\mu^{\prime}, \frac{\theta^{\prime}}{t}\right)}}+\left\|\Phi_{I, 2}^{(\nu)}\left[R_{\frac{1}{\nu}} h_{I B}+h_{I I}, H^{(\nu)}\left[h_{I}\right]-H^{(\nu)}\left[f_{I}\right]\right]\right\|_{X_{I I, 0}^{\left(\mu^{\prime}, \frac{\theta^{\prime}}{t}\right)}} \\
& \lesssim \frac{C_{E}}{(t-s)^{\frac{1}{2}}}\left(1+\left\|f_{I}\right\|_{X_{I, 1}^{\left(0, \frac{1}{2^{7}}, 0\right)}(s)}\right)\left(1+\left\|h_{I}\right\|_{X_{I, 1}^{\left(0, \frac{1}{2^{7}}, 0\right)}}\right)\left\|f_{I}-h_{I}\right\|_{X_{I, 0}^{\left(0, \frac{1}{2^{7}}, 0\right)}(s)} .
\end{aligned}
$$

We omit the details. For $\Phi_{I, 3}^{(\nu)}\left[\omega_{E}+R_{1 / \nu} w_{B}, H^{(\nu)}\left[f_{I}\right]\right]$ we use Lemma 3.10 and Proposition 3.11 to get

$$
\begin{aligned}
& \left\|\Phi_{I, 3}^{(\nu)}\left[\omega_{E}+R_{\frac{1}{\nu}} w_{B}, H^{(\nu)}\left[f_{I}\right]\right]\right\|_{X_{I I, 1}^{\left(\mu^{\prime}, \frac{\theta^{\prime}}{t}\right)}} \\
\lesssim & \left(1+\left(\frac{\nu s}{d_{E}^{3}}\right)^{\frac{1}{2}}+d_{E}^{-1} e^{-\frac{1}{2^{7} \nu s}} d_{E}^{2}\right)\left(\left\|w_{B}\right\|_{Z_{B}}+\left\|\omega_{E}\right\|_{Z_{E}}\right)\left\|\nabla\left(\chi_{8 d_{E}}^{c} H^{(\nu)}\left[f_{I}\right](s)\right)\right\|_{W^{1,2} \cap L^{\frac{4}{3}}} \\
\lesssim & C_{E}\left(\left\|\nabla \Omega\left[f_{I}\right]\right\|_{L^{1}\left(0, s ; W^{1,2} \cap L^{\frac{4}{3}}\right)}+\left\|\Omega\left[f_{I}\right]\right\|_{L^{1}\left(0, s ; L^{1}\right)}\right)
\end{aligned}
$$

for $0<\nu<d_{E}^{5}$ and $0<s<d_{E}$, where $\Omega\left[f_{I}\right]$ is defined by (4.29). Thus (4.30), (4.34), and (4.38) yield

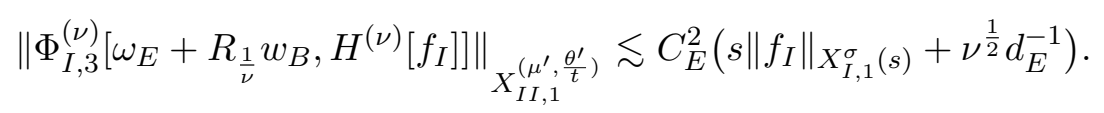

By the same argument we have

$$
\left\|\Phi_{I, 3}^{(\nu)}\left[R_{\frac{1}{\nu}} f_{I B}+f_{I I}, H^{(\nu)}\left[f_{I}\right]\right]\right\|_{X_{I I, 1}^{\left(\mu^{\prime}, \frac{\theta^{\prime}}{t}\right)}} \lesssim C_{E}\left\|f_{I}\right\|_{X_{I, 1}^{\left(0, \frac{1}{2^{7}}, 0\right)}(s)}\left(s\left\|f_{I}\right\|_{X_{I, 1}^{\sigma}(s)}+\nu^{\frac{1}{2}} d_{E}^{-1}\right) .
$$


Next, as in the proof of (4.36), we have from Lemma 3.10, Proposition 3.14, and $\left\|\omega_{E}\right\|_{Z_{E}}+\left\|w_{B}\right\|_{Z_{B}} \leq C_{E}$,

$$
\begin{array}{r}
\left\|\Phi_{I, 3}^{(\nu)}\left[\omega_{E}+R_{\frac{1}{\nu}} w_{B}, H^{(\nu)}\left[f_{I}\right]-H^{(\nu)}\left[h_{I}\right]\right]\right\|_{X_{I I, 0}^{\left(\mu^{\prime}, \frac{\theta^{\prime}}{t}\right)}} \lesssim C_{E}\left\|\nabla\left(\chi_{8 d_{E}}^{c} H^{(\nu)}\left[f_{I}\right](s)-\chi_{8 d_{E}}^{c} H^{(\nu)}\left[h_{I}\right](s)\right)\right\|_{L^{2} \cap L^{\frac{4}{3}}} \\
\lesssim C_{E}\left(\nu^{-\frac{1}{4}}\left(\left\|\nabla \Omega\left[f_{I}\right]\right\|_{L^{1}\left(0, s ; W^{1,2} \cap L^{\frac{4}{3}}\right)}+\left\|\Omega\left[f_{I}\right]\right\|_{L^{1}\left(0, s ; L^{1}\right)}\right)\left\|f_{I}-h_{I}\right\|_{X_{I, 0}^{\sigma}(s)}\right. \\
\left.+\left\|\nabla \Omega\left[f_{I}\right]-\nabla \Omega\left[h_{I}\right]\right\|_{L^{1}\left(0, s ; L^{2} \cap L^{\frac{4}{3}}\right)}+\left\|\Omega\left[f_{I}\right]-\Omega\left[h_{I}\right]\right\|_{L^{1}\left(0, s ; L^{1}\right)}\right) .
\end{array}
$$

Hence, the estimates (4.30), (4.34), (4.35), and (4.41) yield

$$
\left\|\Phi_{I, 3}^{(\nu)}\left[\omega_{E}+R_{\frac{1}{\nu}} w_{B}, H^{(\nu)}\left[f_{I}\right]-H^{(\nu)}\left[h_{I}\right]\right]\right\|_{X_{I I, 0}^{\left(\mu^{\prime}, \frac{\theta^{\prime}}{t}\right)}} \lesssim C_{E}^{2}\left(1+\nu^{-\frac{1}{4}}\left\|f_{I}\right\|_{X_{I, 1}^{\left(0, \frac{1}{2^{7}}, 0\right)}(s)}\right)\left\|f_{I}-h_{I}\right\|_{X_{I, 0}^{\sigma}(s)} .
$$

Similarly, we have

$$
\begin{aligned}
& \left\|\Phi_{I, 3}^{(\nu)}\left[R_{\frac{1}{\nu}} f_{I B}+f_{I I}, H^{(\nu)}\left[f_{I}\right]\right]-\Phi_{I, 3}^{(\nu)}\left[R_{\frac{1}{\nu}} h_{I B}+h_{I I}, H^{(\nu)}\left[h_{I}\right]\right]\right\|_{X_{I I, 0}^{\left(\mu^{\prime}, \frac{\theta^{\prime}}{t}\right)}} \\
\leq & \left\|\Phi_{I, 3}^{(\nu)}\left[R_{\frac{1}{\nu}} f_{I B}-R_{\frac{1}{\nu}} h_{I B}+f_{I I}-h_{I I}, H^{(\nu)}\left[f_{I}\right]\right]\right\|_{X_{I I, 0}^{\left(\mu^{\prime}, \frac{\theta^{\prime}}{t}\right)}}+\left\|\Phi_{I, 3}^{(\nu)}\left[R_{\frac{1}{\nu}} h_{I B}+h_{I I}, H^{(\nu)}\left[h_{I}\right]-H^{(\nu)}\left[f_{I}\right]\right]\right\|_{X_{I I, 0}^{\left(\mu^{\prime}, \frac{\theta^{\prime}}{t}\right)}} \\
\lesssim & C_{E}\left(1+\nu^{-\frac{1}{4}}\left\|f_{I}\right\|_{X_{I, 1}^{\left(0, \frac{1}{2^{7}}, 0\right)}(s)}\right)\left(1+\left\|h_{I}\right\|_{X_{I, 1}^{\left(0, \frac{1}{2^{7}}, 0\right)}(s)}\right)\left\|f_{I}-h_{I}\right\|_{X_{I, 0}^{\sigma}(s)} .
\end{aligned}
$$

Then (4.21) follows from (4.23), (4.25), (4.26), (4.31), (4.32), (4.39), (4.40), and from the assumption $\left\|f_{I}\right\|_{X_{I, 1}^{\left(0,1 / 2^{7}, 0\right)}(t)} \leq \nu^{1 / 4}$. Similarly, (4.22) follows from (4.24), (4.27), (4.36), (4.37), (4.42), (4.43), and from the assumptions $\left\|f_{I}\right\|_{X_{I, 1}^{\left(0,1 / 2^{7}, 0\right)}(t)} \leq \nu^{1 / 4}$ and $\left\|h_{I}\right\|_{X_{I, 1}^{\left(0,1 / 2^{7}, 0\right)}(t)} \leq \nu^{1 / 4}$. The proof is complete.

Lemma 4.8 Assume that $0<\mu<d_{E} / 2,0<\theta \leq 2^{-8}$, and $0<s<t<T_{B}$, where $T_{B}$ is the number in Theorem 4.4. Let $0<\nu<d_{E}^{2}$. Then

$$
\left\|F_{I I}^{(\nu)}(t, s)\right\|_{X_{I, 1}^{\left(\mu, \frac{\theta}{t}\right)}} \lesssim \frac{\nu^{\frac{1}{2}}}{d_{E}}\left\|\omega_{E}\right\|_{Z_{E}} .
$$

Proof. From (3.6) and (3.13) it is easy to see $\nu\left\|e^{\nu(t-s) \Delta_{N}} \Delta \omega_{E}(s)\right\|_{X_{I I, 1}^{(\mu, \theta / t)}} \lesssim \nu\left\|\omega_{E}\right\|_{Z_{E}}$. For the term $\Phi_{I}^{(\nu)}\left[R_{1 / \nu} w_{B}, \omega_{E}\right]=\Phi_{I, 3}^{(\nu)}\left[R_{1 / \nu} w_{B}, \omega_{E}\right]$ we have from (3.66) that $\left\|\Phi_{I}^{(\nu)}\left[R_{1 / \nu} w_{B}(s), \omega_{E}(s)\right](t-s)\right\|_{X_{I I, 1}^{(\mu, \theta / t)}}=$ $\left\|\Phi_{I, 3}^{(\nu)}\left[R_{1 / \nu} w_{B}(s), \omega_{E}(s)\right](t-s)\right\|_{X_{I I, 1}^{(\mu, \theta / t)}}$ is bounded from above by $\nu^{1 / 2} d_{E}^{-1}\left\|w_{B}\right\|_{Z_{B}}\left\|\omega_{E}\right\|_{Z_{E}}$. Here we have used $0<s<t<d_{E}$ and $0<\nu<d_{E}^{2}$. This completes the proof, since $\left\|w_{B}\right\|_{Z_{B}} \leq 1$.

Let $\gamma_{0}^{\prime} \in\left(0, T_{1}\right)$ and set

$$
\gamma_{k+1}^{\prime}=\gamma_{k}^{\prime}\left(1-(k+2)^{-2}\right), \quad \gamma^{\prime}=\lim _{k \rightarrow \infty} \gamma_{k}^{\prime}=\gamma_{0}^{\prime} \Pi_{k=0}^{\infty}\left(1-(k+2)^{-2}\right)>0 .
$$

The next lemma is a counterpart of Lemma 4.3, but the argument becomes more complicated. Roughly speaking, we aim the uniform bound of $\left\{w_{I}^{(k)}\right\}$ in $X_{I B, 1}^{(\mu, \rho)} \times X_{I I, 1}^{(\mu, \theta)}$, while the convergence estimate will be established in a weaker topology of $X_{I B, 0}^{(\mu, \rho)} \times X_{I I, 0}^{(\mu, \theta)}$. 
Lemma 4.9 Let $0<\nu<d_{E}^{5}$. Let $\mu_{0}^{\prime}=d_{E} / 2, \rho_{0}^{\prime}=2^{-5}, \theta_{0}^{\prime}=2^{-8}$, and $\sigma_{0}^{\prime}=\left(\mu_{0}^{\prime}, \rho_{0}^{\prime}, \theta_{0}^{\prime}\right)$. Set

$$
\begin{aligned}
& \lambda_{k}=\sup _{\frac{1}{2} \leq \kappa<1} \sup _{0<t<\gamma_{k}^{\prime}(1-\kappa)}\left\|w_{I}^{(k)}\right\|_{X_{I, 1}^{\kappa \sigma_{0}^{\prime}(t)}}\left(\frac{\gamma_{k}^{\prime}(1-\kappa)}{t}-1\right)^{\frac{1}{8}}, \quad \eta_{k}=\sup _{0<t<\frac{1}{2} \gamma_{k}^{\prime}}\left\|w_{I}^{(k)}\right\|_{X_{I, 1}^{\sigma_{0}^{\prime} / 4}(t)}, \\
& \zeta_{k}=\sup _{\frac{1}{2} \leq \kappa<1} \sup _{0<t<\gamma_{k}^{\prime}(1-\kappa)}\left\|w_{I}^{(k+1)}-w_{I}^{(k)}\right\|_{X_{I, 0}^{\kappa \sigma_{0}^{\prime}(t)}}\left(\frac{\gamma_{k}^{\prime}(1-\kappa)}{t}-1\right)^{\frac{1}{8}} .
\end{aligned}
$$

If $\gamma_{0}^{\prime}>0$ is sufficiently small then it follows that $\lambda_{k} \leq \nu^{\frac{1}{2}}, \eta_{k} \leq \nu^{\frac{1}{2}}$, and $\zeta_{k} \leq \delta^{k} \zeta_{0}$ for all $k \in \mathbb{N}$ and for some $\delta \in(0,1)$. Moreover, $\gamma_{0}^{\prime}$ is taken so that $\gamma_{0}^{\prime} \geq c_{0}^{\prime} d_{E}$, where $c_{0}^{\prime}>0$ depends only on $\|b\|_{Y_{E}}$.

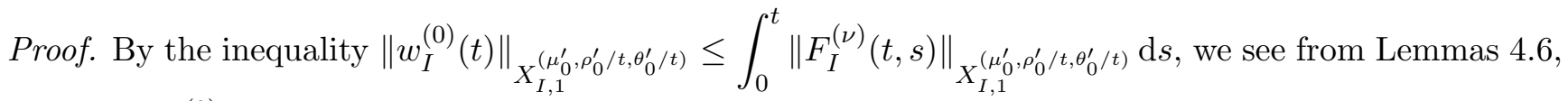

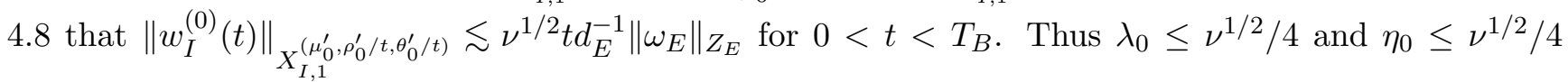
hold for $0<\gamma_{0}^{\prime}=c_{0}^{\prime} d_{E}$ with sufficiently small $c_{0}^{\prime}>0$ depending only on $\|b\|_{Y_{E}}$. Now let us assume that $\lambda_{i} \leq \nu^{1 / 2}$ and $\eta_{i} \leq \nu^{1 / 2}$ for $i=0,1, \cdots, k$. Then for $0<\tilde{t} \leq t<\gamma_{k+1}^{\prime}(1-\kappa)$ and $1 / 4 \leq \kappa<1$ we have from (4.19) with $h_{I}=0, j=1$, and (4.21),

$$
\begin{aligned}
& \left\|w_{I}^{(k+1)}(\tilde{t})\right\|_{\left.X_{I, 1}^{\kappa\left(\mu_{0}^{\prime}\right.}, \frac{\rho_{0}^{\prime}}{t}, \frac{\theta_{0}^{\prime}}{t}\right)} \lesssim \frac{C_{E}}{d_{E}} \int_{0}^{\tilde{t}} \frac{\left\|w_{I}^{(k)}\right\|_{X_{I, 1}^{\kappa(s) \sigma_{0}^{\prime}}(s)}}{\kappa(s)-\kappa} \mathrm{d} s+\frac{C_{E}^{2}}{d_{E}^{\frac{1}{2}}} \int_{0}^{\tilde{t}} \frac{\left\|w_{I}^{(k)}\right\|_{X_{I, 1}^{\kappa(s) \sigma_{0}^{\prime}}(s)}}{(\tilde{t}-s)^{\frac{1}{2}}(\kappa(s)-\kappa)^{\frac{1}{2}}} \mathrm{~d} s \\
& +\int_{0}^{\tilde{t}}\left(\frac{1}{\nu^{\frac{1}{2}}(\tilde{t}-s)^{\frac{1}{2}}(\kappa(s)-\kappa)^{\frac{1}{4}}}+\frac{\nu^{\frac{1}{4}} d_{E}^{\frac{1}{2}}}{(\tilde{t}-s)^{\frac{1}{4}}(\kappa(s)-\kappa)^{\frac{1}{2}}}\right)\left\|w_{I}^{(k)}\right\|_{X_{I, 1}^{\kappa(s) \sigma_{0}^{\prime}(s)}}^{2} \mathrm{~d} s+\frac{C_{E}^{2} \nu^{\frac{1}{2}} \tilde{t}}{d_{E}} .
\end{aligned}
$$

Here $\kappa(s)$ is chosen so that $\kappa<\kappa(s)$ and $s<\gamma_{k}^{\prime}(1-\kappa(s))$. First we take $1 / 2 \leq \kappa<1$ and $\kappa(s)=$ $2^{-1}\left(1-s / \gamma_{k+1}^{\prime}+\kappa\right)$. Then we have from $\left\|w_{I}^{(k)}\right\|_{X_{I, 1}^{\kappa(s) \sigma_{0}^{\prime}(s)}} \leq\left(\gamma_{k}^{\prime}(1-\kappa(s)) / s-1\right)^{-1 / 8} \lambda_{k}$ and $\gamma_{k+1}^{\prime}<\gamma_{k}^{\prime}$,

$$
\begin{aligned}
& \int_{0}^{\tilde{t}} \frac{\left\|w_{I}^{(k)}\right\|_{X_{I, 1}^{\kappa(s) \sigma_{0}^{\prime}}(s)}}{\kappa(s)-\kappa} \mathrm{d} s \lesssim \gamma_{k+1}^{\prime} \lambda_{k} \tilde{t}^{\frac{1}{8}} \int_{0}^{\tilde{t}} \frac{1}{\left(\gamma_{k+1}^{\prime}(1-\kappa)-s\right)^{\frac{9}{8}}} \mathrm{~d} s \lesssim \frac{\gamma_{0}^{\prime} \nu^{\frac{1}{2}} t^{\frac{1}{8}}}{\left(\gamma_{k+1}^{\prime}(1-\kappa)-t\right)^{\frac{1}{8}}}, \\
& \int_{0}^{\tilde{t}} \frac{\left\|w_{I}^{(k)}\right\|_{X_{I, 1}^{\kappa(s) \sigma_{0}^{\prime}}(s)}}{(\tilde{t}-s)^{\frac{1}{2}}(\kappa(s)-\kappa)^{\frac{1}{2}}} \mathrm{~d} s \lesssim\left(\gamma_{k+1}^{\prime}\right)^{\frac{1}{2}} \lambda_{k} \tilde{t}^{\frac{1}{8}} \int_{0}^{\tilde{t}} \frac{1}{(\tilde{t}-s)^{\frac{1}{2}}\left(\gamma_{k+1}^{\prime}(1-\kappa)-s\right)^{\frac{5}{8}}} \mathrm{~d} s \lesssim \frac{\gamma_{0}^{\prime \frac{1}{2}} \nu^{\frac{1}{2}} t^{\frac{1}{8}}}{\left(\gamma_{k+1}^{\prime}(1-\kappa)-t\right)^{\frac{1}{8}}},
\end{aligned}
$$

and similarly,

$$
\int_{0}^{\tilde{t}}\left(\frac{1}{\nu^{\frac{1}{2}}(\tilde{t}-s)^{\frac{1}{2}}(\kappa(s)-\kappa)^{\frac{1}{4}}}+\frac{\nu^{\frac{1}{4}} d_{E}^{\frac{1}{2}}}{(\tilde{t}-s)^{\frac{1}{4}}(\kappa(s)-\kappa)^{\frac{1}{2}}}\right)\left\|w_{I}^{(k)}\right\|_{X_{I, 1}^{\kappa(s) \sigma_{0}^{\prime}}(s)}^{2} \mathrm{~d} s \lesssim \frac{\gamma_{0}^{\prime \frac{1}{4}} \nu^{\frac{1}{2}} t^{\frac{3}{8}}}{\left(\gamma_{k+1}^{\prime}(1-\kappa)-t\right)^{\frac{1}{8}}} .
$$

These estimates yield $\lambda_{k+1} \leq \nu^{\frac{1}{2}}$ if $0<\nu<d_{E}^{5}$ and $\gamma_{0}^{\prime}=c_{0}^{\prime} d_{E}$, where $c_{0}^{\prime}>0$ is small enough (but depending only on $\left.\|b\|_{Y_{E}}\right)$. Next we take $\kappa=1 / 4$ and $\kappa(s)=2^{-1}\left(3 / 2-s / \gamma_{k+1}^{\prime}\right)$ in (4.46). Then $\kappa(s)-\kappa \geq 1 / 4$ for $s<\gamma_{k+1}^{\prime} / 2$, and thus, when $0<\tilde{t} \leq t<\gamma_{k+1}^{\prime} / 2$ we have

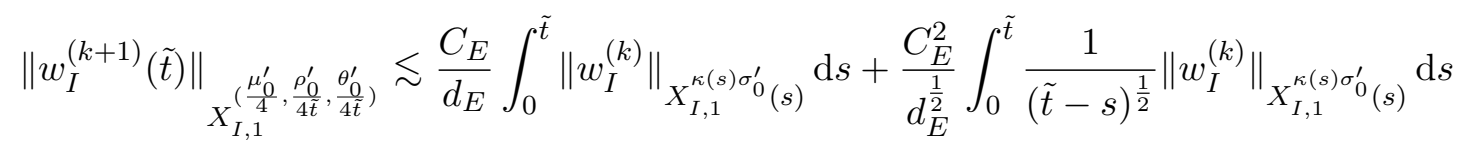

$$
\begin{aligned}
& +\int_{0}^{\tilde{t}}\left(\frac{1}{\nu^{\frac{1}{2}}(\tilde{t}-s)^{\frac{1}{2}}}+\frac{\nu^{\frac{1}{4}} d_{E}^{\frac{1}{2}}}{(\tilde{t}-s)^{\frac{1}{4}}}\right)\left\|w_{I}^{(k)}\right\|_{X_{I, 1}^{\kappa(s) \sigma_{0}^{\prime}(s)}}^{2} \mathrm{~d} s+C_{E}^{2} \frac{\nu^{\frac{1}{2}} \tilde{t}}{d_{E}} \lesssim C_{E}^{2}\left(\frac{\nu t}{d_{E}}\right)^{\frac{1}{2}} .
\end{aligned}
$$


This proves $\eta_{k+1} \leq \nu^{1 / 2}$ if $0<\nu<d_{E}^{5}$ and $\gamma_{0}^{\prime}=c_{0}^{\prime} d_{E}$. By the induction on $k$ we have proved that $\lambda_{k} \leq \nu^{1 / 2}$ and $\eta_{k} \leq \nu^{1 / 2}$ for all $k \in \mathbb{N} \cup\{0\}$. To estimate $\zeta_{k}$ we use (4.19) and (4.22). Then it follows that for $0<\tilde{t} \leq t<\gamma_{k+1}^{\prime}(1-\kappa)$ and $1 / 2 \leq \kappa<1$,

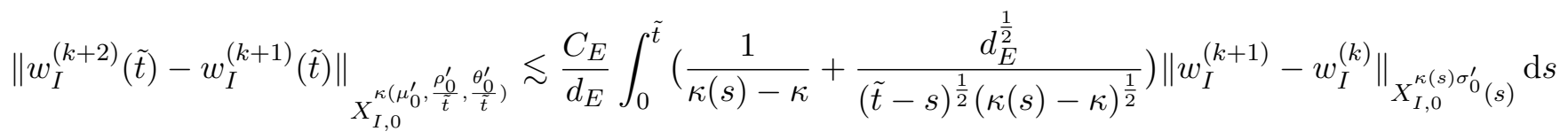

$$
\begin{aligned}
& +\sum_{j=0,1} \int_{0}^{\tilde{t}}\left(\frac{\nu^{\frac{1}{4}} d_{E}^{\frac{1}{2}}}{(\tilde{t}-s)^{\frac{1}{4}}(\kappa(s)-\kappa)^{\frac{1}{2}}}+\frac{1}{\nu^{\frac{1}{2}}(\tilde{t}-s)^{\frac{1}{2}}(\kappa(s)-\kappa)^{\frac{1}{4}}}\right)\left\|w_{I}^{(k+j)}\right\|_{X_{I, 1}^{\kappa(s) \sigma_{0}^{\prime}(s)}}\left\|w_{I}^{(k+1)}-w_{I}^{(k)}\right\|_{X_{I, 0}^{\kappa(s) \sigma_{0}^{\prime}(s)}} \mathrm{d} s \\
& +C_{E}^{2} \int_{0}^{\tilde{t}}(\tilde{t}-s)^{-\frac{1}{2}}\left\|w_{I}^{(k+1)}-w_{I}^{(k)}\right\|_{X_{I, 0}^{\kappa(s) \sigma_{0}^{\prime}(s)}} \mathrm{d} s .
\end{aligned}
$$

Again let us take $\kappa(s)=2^{-1}\left(1-s / \gamma_{k+1}^{\prime}+\kappa\right)$. Then, since $\left\|w_{I}^{(k+1)}-w_{I}^{(k)}\right\|_{X_{I, 0}^{\kappa(s) \sigma_{0}^{\prime}(s)}}$ and $\left\|w_{I}^{(k+j)}\right\|_{X_{I, 1}^{\kappa(s) \sigma_{0}^{\prime}(s)}}$ are bounded from above by $\left(\gamma_{k}^{\prime}(1-\kappa(s)) / s-1\right)^{-1 / 8} \zeta_{k}$ and $\left(\gamma_{k+j}^{\prime}(1-\kappa(s)) / s-1\right)^{-1 / 8} \lambda_{k+j}$, respectively, we have

$$
\left\|w_{I}^{(k+2)}(\tilde{t})-w_{I}^{(k+1)}(\tilde{t})\right\|_{X_{I, 0}^{\kappa\left(\mu_{0}^{\prime}, \frac{\rho_{0}^{\prime}}{t}, \frac{\theta_{0}^{\prime}}{t}\right)}} \lesssim\left(\frac{t}{\gamma_{k+1}(1-\kappa)-t}\right)^{\frac{1}{8}} C_{E}^{2}\left(\frac{\gamma_{0}^{\prime}}{d_{E}}\right)^{\frac{1}{2}} \zeta_{k} .
$$

Hence, $\zeta_{k+1} \leq \delta \zeta_{k}$ holds for some $\delta \in(0,1)$ if $\gamma_{0}^{\prime}=c_{0}^{\prime} d_{E}$ with sufficiently small $c_{0}^{\prime}>0$ (depending only on $\left.\|b\|_{Y_{E}}\right)$ and $0<\nu<d_{E}^{5}$. This completes the proof.

Theorem 4.10 There is $T_{I} \in\left(0, T_{0}\right)$ such that (4.14) admits the unique solution $w_{I}$ which belongs to the space $C\left(\left[0, T_{I}\right) ; X_{I, 1}^{\left(\mu_{I}, \rho_{I} / T_{I}, \theta_{I} / T_{I}\right)}\right)$ with $\mu_{I}=d_{E} / 8, \rho_{I}=2^{-7}, \theta_{I}=2^{-10}$, and satisfies the estimate

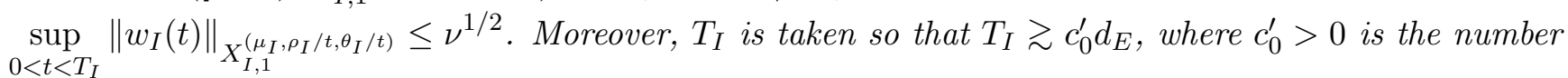
in Lemma 4.9 .

Proof. By Lemma 4.9 we observe that $\left\{w_{I}^{(k)}\right\}_{k=0}^{\infty}$ is a Cauchy sequence in the Banach space endowed with the norm $\|F\|=\sup _{1 / 2 \leq \kappa<1} \sup _{0<t<\gamma^{\prime}(1-\kappa)}\left(\frac{\gamma^{\prime}(1-\kappa)}{t}-1\right)^{1 / 8}\|F\|_{X_{I, 0}^{\kappa \sigma_{0}^{\prime}(t)}}$. Let $w_{I}$ be the limit of $\left\{w_{I}^{(k)}\right\}_{k=0}^{\infty}$ in this Banach space. Then again by Lemma 4.9 we also have

$$
\sup _{\frac{1}{2} \leq \kappa<1} \sup _{0<t<\gamma^{\prime}(1-\kappa)}\left(\frac{\gamma^{\prime}(1-\kappa)}{t}-1\right)^{\frac{1}{8}}\left\|w_{I}\right\|_{X_{I, 1}^{\kappa \sigma_{0}^{\prime}(t)}} \leq \nu^{\frac{1}{2}}, \quad \sup _{0<t<\frac{1}{2} \gamma^{\prime}}\left\|w_{I}\right\|_{X_{I, 0}^{\sigma_{0}^{\prime} / 4}(t)} \leq \nu^{\frac{1}{2}} .
$$

It is easy to see that $w_{I}$ solves the integral equation (4.14). Moreover, $w_{I I}=H^{(\nu)}\left[w_{I}\right]$ holds, since $z_{I I}=w_{I I}-H^{(\nu)}\left[w_{I}\right]$ satisfies the integral equations for

$$
\partial_{t} z_{I I}-\nu \Delta z_{I I}=-J\left(\omega_{E}+R_{\frac{1}{\nu}} w_{B}+R_{\frac{1}{\nu}} w_{I B}+w_{I I}\right) \cdot \nabla\left(\chi_{4 d_{E}} z_{I I}\right),\left.\quad \partial_{2} z_{I I}\right|_{x_{2}=0}=0,\left.\quad z_{I I}\right|_{t=0}=0 .
$$

It is not difficult to show $z_{I I}=0$ from (4.48). Hence $w_{I I}$ satisfies the heat-transport equation (HT) with

$$
u=J\left(\omega_{E}+R_{\frac{1}{\nu}} w_{B}+R_{\frac{1}{\nu}} w_{I B}+w_{I I}\right), \quad f=-J\left(R_{\frac{1}{\nu}} w_{B}+R_{\frac{1}{\nu}} w_{I B}+w_{I I}\right) \cdot \nabla \omega_{E}+\nu \Delta \omega_{E} .
$$

Then the definition of $\Lambda_{I}^{(\nu)}\left(t, s, w_{I}\right)$ implies $\omega_{I}=R_{1 / \nu} w_{I B}+w_{I I}$ solves $\left(\mathrm{V}_{\mathrm{I}_{\nu}}\right)$. The proof is complete. 


\subsection{Convergence of $w_{B}$ to the solution of $\left(\mathrm{V}_{\mathrm{p}}\right)$}

In this section we prove the convergence of $w_{B}=w_{B}^{(\nu)}$ in Theorem 4.4 to the solution of the vorticity equations for the Prandtl equations, i.e., Eq. $\left(\mathrm{V}_{\mathrm{p}}\right)$, at the limit $\nu \rightarrow 0$. To this end we first solve $\left(\mathrm{V}_{\mathrm{p}}\right)$, where its proof is almost same as in Theorem 4.4. Let $\left\{e^{t A}\right\}_{t \geq 0}$ be the semigroup for the one-dimensional heat equations in $\left\{\left(t, X_{2}\right) \mid t \geq 0, \quad X_{2} \in \mathbb{R}_{+}\right\}$subject to the homogeneous Neumann boundary condition. Then the integral equation for $\left(\mathrm{V}_{\mathrm{p}}\right)$ is written as

$$
\begin{aligned}
w_{P}(t)=- & \int_{0}^{t} e^{(t-s) A} B^{(0)}\left(R_{0} \omega_{E}+w_{P}, w_{P}\right) \mathrm{d} s \\
& +\int_{0}^{t} e^{(t-s) A}\left(N^{(0)}\left(R_{0} \omega_{E}+w_{P}, w_{P}\right) \mathcal{H}_{\left\{X_{2}=0\right\}}^{1}+N\left(\omega_{E}, \omega_{E}\right) \mathcal{H}_{\left\{X_{2}=0\right\}}^{1}\right) \mathrm{d} s,
\end{aligned}
$$

where

$$
\begin{array}{ll}
B^{(0)}\left(R_{0} \omega_{E}, h\right)=\lim _{\nu \rightarrow 0} B^{(\nu)}\left(R_{\nu} \omega_{E}, h\right), & B^{(0)}(f, h)=\lim _{\nu \rightarrow 0} B^{(\nu)}(f, h), \\
N^{(0)}\left(R_{0} \omega_{E}, h\right)=\lim _{\nu \rightarrow 0} N^{(\nu)}\left(R_{\nu} \omega_{E}, h\right), & N^{(0)}(f, h)=\lim _{\nu \rightarrow 0} N^{(\nu)}(f, h) .
\end{array}
$$

Here the limits in (4.50) - (4.51) are taken in the formal sense for a while. It is easy to see that, as desired, $B^{(0)}\left(R_{0} \omega_{E}, h\right)$ is equal to $v_{E} \cdot \nabla_{X} h$ with $v_{E}$ given in (2.4) and (2.5), and that

$$
\begin{aligned}
& B^{(0)}(f, h)(X)=\int_{X_{2}}^{\infty} f\left(x_{1}, Y_{2}\right) \mathrm{d} Y_{2} \partial_{1} h-\partial_{1}\left(\int_{0}^{X_{2}} Y_{2} f\left(x_{1}, Y_{2}\right) \mathrm{d} Y_{2}+X_{2} \int_{X_{2}}^{\infty} f\left(x_{1}, Y_{2}\right) \mathrm{d} Y_{2}\right) \partial_{X_{2}} h(X) \\
& N^{(0)}\left(R_{0} \omega_{E}+f, h\right)\left(x_{1}\right)=\int_{0}^{\infty} B^{(0)}\left(R_{0} \omega_{E}+f, h\right)\left(x_{1}, Y_{2}\right) \mathrm{d} Y_{2} .
\end{aligned}
$$

Here $X=\left(x_{1}, X_{2}\right)$. We set $\varphi_{P}^{(\mu, \rho)}\left(\xi_{1}, X_{2}\right)=\exp \left(\frac{\mu\left|\xi_{1}\right|}{4}+\rho X_{2}^{2}\right)$ with $\mu, \rho \geq 0$, and introduce the norm

$$
\|f\|_{X_{P}^{(\mu, \rho)}}=\sum_{k=0,1}\left(\left\|\varphi_{P}^{(\mu, \rho)} X_{2}^{\frac{k}{2}}\left\langle\xi_{1}\right\rangle^{2} \hat{f}\left(\xi_{1}, X_{2}\right)\right\|_{L_{\xi_{1}}^{2} L_{X_{2}}^{1+k}}+\left\|\varphi_{P}^{(\mu, \rho)} X_{2}^{1+\frac{k}{2}}\left\langle\xi_{1}\right\rangle \partial_{X_{2}} \hat{f}\left(\xi_{1}, X_{2}\right)\right\|_{L_{\xi_{1}}^{2} L_{X_{2}}^{1+k}}\right) .
$$

Lemma 4.11 Assume that $0<2^{-1}\left(\mu-\mu^{\prime}\right)<\mu^{\prime}<\mu<1,0<\rho^{\prime}<\rho \leq 2^{-4}$, and $0<s<t<T_{0}$. Then it follows that

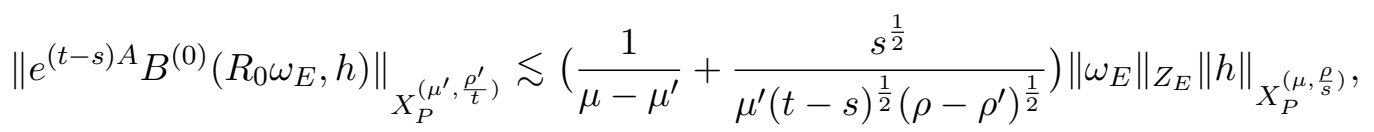

$$
\begin{aligned}
& \left\|e^{(t-s) A} B^{(0)}(f, h)\right\|_{X_{P}^{\left(\mu^{\prime}, \frac{\rho^{\prime}}{t}\right)}} \lesssim\left(\frac{1}{\mu-\mu^{\prime}}+\frac{s^{\frac{1}{2}}}{\mu^{\prime}(t-s)^{\frac{1}{2}}\left(\rho-\rho^{\prime}\right)^{\frac{1}{2}}}\right)\|f\|_{X_{P}^{(\mu, 0)}}\|h\|_{X_{P}^{\left(\mu, \frac{\rho}{s}\right)}}, \\
& \left\|e^{(t-s) A}\left\{N^{(0)}\left(R_{0} \omega_{E}, h\right) \mathcal{H}_{\left\{X_{2}=0\right\}}^{1}\right\}\right\|_{X_{P}^{\left(\mu^{\prime}, \frac{\rho^{\prime}}{t}\right)}} \lesssim \frac{1}{\mu-\mu^{\prime}}\left\|\omega_{E}\right\|_{Z_{E}}\|h\|_{X_{P}^{\left(\mu, \frac{\rho}{s}\right)}},
\end{aligned}
$$

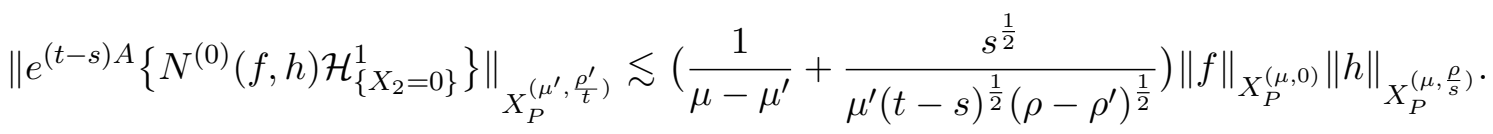

Lemma 4.11 is obtained by combining Lemmas 3.4 and 3.5 with Lemma 7.3 in the appendix if one takes the limit $\nu \rightarrow 0$ of the estimates in these lemmas. The details are left to the reader. 
Theorem 4.12 There is $T_{P} \in\left(0, T_{0}\right)$ such that (4.49) admits the unique solution $w_{P}$ which belongs to the space $C\left(\left[0, T_{P}\right) ; X_{P}^{\left(\mu_{P}, \rho_{P} / T_{P}\right)}\right)$ with $\mu_{P}=d_{E} / 2, \rho_{P}=2^{-5}$, and satisfies $\sup _{0<t<T_{P}}\left\|w_{P}(t)\right\|_{X_{P}^{\left(\mu_{P}, \rho_{P} / t\right)}} \leq 1$. Moreover, $T_{P}$ is taken so that $T_{P} \geq c_{P} d_{E}$, where $c_{P}>0$ depends only on $\|b\|_{Y_{E}}$.

Proof. The proof is carried out in the same way as in Theorem 4.4 by using the estimates for the bilinear forms given by Lemma 4.11. So we omit the details here. The proof is complete.

Theorem 4.13 Let $w_{B}=w_{B}^{(\nu)}$ and $w_{P}$ be the functions obtained by Theorem 4.4 and Theorem 4.12, respectively. Then there is $T_{P}^{\prime}>0$ satisfying

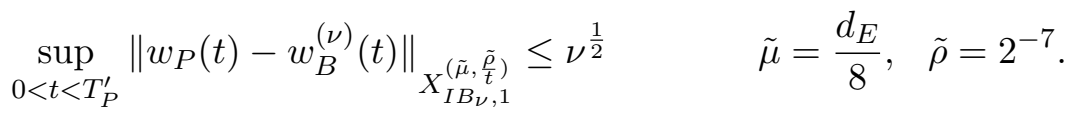

Moreover, $T_{P}^{\prime}$ is taken so that $T_{P}^{\prime} \geq c d_{E}$, where $c>0$ depends only on $\|b\|_{Y_{E}}$.

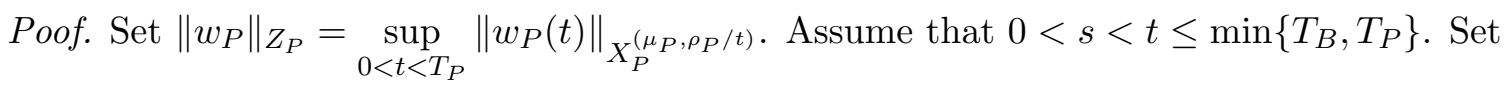

$$
\begin{aligned}
I(t)= & \sum_{i=1,2} \int_{0}^{t}\left(\Phi_{B, i}^{(\nu)}\left[R_{\nu} \omega_{E}+w_{P}, w_{P}\right](t-s)-\Phi_{B, i}^{(\nu)}\left[R_{\nu} \omega_{E}+w_{B}, w_{B}\right](t-s)\right) \mathrm{d} s \\
I I(t)=- & \int_{0}^{t}\left(e^{(t-s) A} B^{(0)}\left(R_{0} \omega_{E}+w_{P}, w_{P}\right)+\Phi_{B, 1}^{(\nu)}\left[R_{\nu} \omega_{E}+w_{P}, w_{P}\right](t-s)\right) \mathrm{d} s \\
& \quad-\int_{0}^{t}\left(e^{(t-s) A}\left(N^{(0)}\left(R_{0} \omega_{E}+w_{P}, w_{P}\right) \mathcal{H}_{\left\{X_{2}=0\right\}}^{1}\right)+\Phi_{B, 2}^{(\nu)}\left[R_{\nu} \omega_{E}+w_{P}, w_{P}\right](t-s)\right) \mathrm{d} s \\
& \quad-\int_{0}^{t}\left(e^{(t-s) A}\left(N\left(\omega_{E}, \omega_{E}\right) \mathcal{H}_{\left\{X_{2}=0\right\}}^{1}\right)+\Phi_{B, 2}^{(\nu)}\left[R_{\nu} \omega_{E}, \omega_{E}\right](t-s)\right) \mathrm{d} s \\
I I I(t)=- & \sum_{i=1,2} \int_{0}^{t}\left(\Upsilon_{i}^{(\nu)}\left[R_{\nu} \omega_{E}+w_{B}, w_{B}\right](s)+\Upsilon_{i}^{(\nu)}\left[R_{\nu} \omega_{E}, R_{\nu} \omega_{E}\right](s)\right) \mathrm{d} s .
\end{aligned}
$$

Thus we have $w_{P}-w_{B}=I+I I+I I I$. As in Lemma 4.3, we set

$$
\zeta=\sup _{\frac{1}{2} \leq \kappa<1} \sup _{0<t<\gamma(1-\kappa)}\left\|w_{P}-w_{B}\right\|_{X_{I B_{\nu}, 1}^{\kappa\left(\frac{d_{E}}{4}, \frac{1}{2^{6}}\right)}(t)}\left(\frac{\gamma(1-\kappa)}{t}-1\right)^{\frac{1}{2}} .
$$

Here $\gamma \in\left(0, \min \left\{T_{B}, T_{P}\right\}\right)$ will be determined later and $\|f\|_{X_{I B_{\nu}, 1}^{(\mu, \rho)}(t)}=\sup _{0<s \leq t}\|f(s)\|_{X_{I B_{\nu}, 1}^{(\mu, \rho / s)}}$. Let $1 / 2 \leq$ $\kappa<1,0<\tilde{t} \leq t<\gamma(1-\kappa)$. Then since $\left\|w_{B}\right\|_{Z_{B_{\nu}}}+\left\|w_{P}\right\|_{Z_{P}} \leq 2$ the estimates (3.36) and (3.49) yield

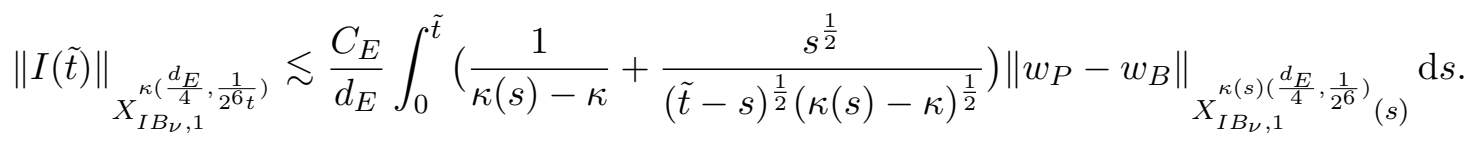

Here we take $\kappa(s)=2^{-1}(1-s / \gamma+\kappa) \in(\kappa, 1)$. Then we have

$$
\|I(\tilde{t})\|_{X_{I B_{\nu}, 1}^{\kappa\left(\frac{d_{E}}{4}, \frac{1}{2^{6} t}\right)}} \lesssim \frac{C_{E} \zeta}{d_{E}} \int_{0}^{\tilde{t}}\left(\frac{1}{\kappa(s)-\kappa}+\frac{s^{\frac{1}{2}}}{(\tilde{t}-s)^{\frac{1}{2}}(\kappa(s)-\kappa)^{\frac{1}{2}}}\right)\left(\frac{s}{\gamma(1-\kappa(s))-s}\right)^{\frac{1}{2}} \mathrm{~d} s \lesssim \frac{C_{E} \gamma^{\frac{1}{2}} t^{\frac{1}{2}} \zeta}{d_{E}^{\frac{1}{2}}(\gamma(1-\kappa)-t)^{\frac{1}{2}}} .
$$


Thus it follows that

$$
\sup _{\frac{1}{2} \leq \kappa<1} \sup _{0<t<\gamma(1-\kappa)}\|I\|_{X_{I B_{\nu}, 1}^{\kappa\left(\frac{d_{E}}{4}, \frac{1}{2^{6}}\right)}(t)}\left(\frac{\gamma(1-\kappa)}{t}-1\right)^{\frac{1}{2}} \leq \frac{\zeta}{2}
$$

if $\gamma=c d_{E}$ with sufficiently small $c>0$ depending only on $\|b\|_{Y_{E}}$. Next we estimate $I I$. Let $0<\mu^{\prime} \leq d_{E} / 4$, $0<\rho^{\prime}<2^{-6}$. It is straightforward to get

$$
\left\|\int_{0}^{t}\left(e^{(t-s) A}\left(N\left(\omega_{E}, \omega_{E}\right) \mathcal{H}_{\left\{X_{2}=0\right\}}^{1}\right)+\Phi_{B, 2}^{(\nu)}\left[R_{\nu} \omega_{E}, \omega_{E}\right](t-s)\right) \mathrm{d} s\right\|_{X_{I B_{\nu}, 1}^{\left(\mu^{\prime}, \frac{\rho^{\prime}}{t}\right)}} \lesssim \nu t^{2}\left\|\omega_{E}\right\|_{Z_{E}}^{2},
$$

and we omit the proof here. For the other terms it suffices to apply Lemma 7.3 with $\mu=d_{E} / 2$ and $\rho=2^{-5}$. Then we conclude that if $0<\nu<d_{E}^{5}$ then $\|I I(t)\|_{X_{I B \nu, 1}^{\left(\mu^{\prime}, \rho^{\prime} / t\right)}} \lesssim \nu^{1 / 2} t d_{E}^{-1}\left(\left\|\omega_{E}\right\|_{Z_{E}}+\left\|w_{P}\right\|_{Z_{P}}\right)^{2}$, i.e.,

$$
\sup _{\frac{1}{2} \leq \kappa<1} \sup _{0<t<\gamma(1-\kappa)}\|I I\|_{X_{I B_{\nu}, 1}^{\kappa\left(\frac{d_{E}}{4}, \frac{1}{\left.2^{6}\right)}\right.}(t)}\left(\frac{\gamma(1-\kappa)}{t}-1\right)^{\frac{1}{2}} \leq \frac{\nu^{\frac{1}{2}}}{4},
$$

where $\gamma=c d_{E}$ with sufficiently small $c>0$ depending only on $\|b\|_{Y_{E}}$. Hence, if we show

$$
\sup _{\frac{1}{2} \leq \kappa<1} \sup _{0<t<\gamma(1-\kappa)}\|I I I\|_{X_{I B_{\nu}, 1}^{\kappa\left(\frac{d}{4}, \frac{1}{2^{6}}\right)}(t)}\left(\frac{\gamma(1-\kappa)}{t}-1\right)^{\frac{1}{2}} \leq \frac{\nu^{\frac{1}{2}}}{4},
$$

then (4.60), (4.62), and (4.63) imply $\zeta \leq \nu^{1 / 2}$, that is, (4.58) holds for $T=\gamma / 4$, where $\gamma=c d_{E}$ and the constant $c>0$ depends only on $\|b\|_{Y_{E}}$. To prove (4.63) we focus only on $\Upsilon_{1}^{(\nu)}\left[w_{B}, w_{B}\right]$, for the other terms are estimated by the same argument. From (3.21) and (3.22) it is not difficult to deduce

$$
\begin{aligned}
& \left\|\varphi_{B_{\nu}}^{\left(\mu^{\prime}, \frac{\rho}{s}\right)}\left\langle\xi_{1}\right\rangle^{2} \chi_{\left\{X_{2} \leq \frac{\mu^{\prime}}{\nu^{\frac{1}{2}}}\right\}} \mathcal{F}\left(B^{(\nu)}(f, h)\right)\right\|_{L_{\xi_{1}}^{2} L_{X_{2}}^{1}} \lesssim \frac{1}{\mu-\mu^{\prime}}\|f\|_{X_{I B_{\nu}, 2}^{(\mu, 0)}}\|h\|_{X_{I B_{\nu}, 2}^{\left(\mu, \frac{\rho}{s}\right)}}, \\
& \left\|\varphi_{B_{\nu}}^{\left(\mu^{\prime}, \frac{\rho}{s}\right)}\left\langle\xi_{1}\right\rangle \chi_{\left\{X_{2} \geq \frac{\mu^{\prime}}{\nu^{\frac{1}{2}}}\right\}} \mathcal{F}\left(B^{(\nu)}(f, h)\right)\right\|_{L_{\xi_{1}}^{2} L_{X_{2}}^{1}} \lesssim\|f\|_{X_{I B_{\nu}, 2}^{(\mu, 0)}\|h\|_{X_{I B_{\nu}, 2}^{\left(\mu, \frac{\rho}{s}\right)}} .}
\end{aligned}
$$

Now we recall that

$$
\begin{aligned}
\mathcal{F}\left(\Upsilon_{1}^{(\nu)}\left[w_{B}, w_{B}\right](s)\right)\left(\xi_{1}, X_{2}\right)=2 \int_{0}^{s} \int_{0}^{\infty}\left(-\nu \xi_{1}^{2}+\nu^{\frac{1}{2}} \xi_{1} \partial_{X_{2}}\right) e^{-\nu(s-\tau) \xi_{1}^{2}} g\left(s-\tau, X_{2}+Y_{2}\right) \\
\cdot\left(\chi_{\left\{Y_{2} \leq \frac{\mu^{\prime}}{\nu^{\frac{1}{2}}}\right\}}+\chi_{\left\{Y_{2} \geq \frac{\mu^{\prime}}{\nu^{\frac{1}{2}}}\right\}}\right) \mathcal{F}\left(B^{(\nu)}\left(w_{B}, w_{B}\right)\right)\left(\xi_{1}, Y_{2}\right) \mathrm{d} Y_{2} \mathrm{~d} \tau,
\end{aligned}
$$

which gives for $l=0,1$,

$$
\begin{aligned}
\left|\left\langle\xi_{1}\right\rangle X_{2}^{l} \partial_{X_{2}}^{l} \mathcal{F}\left(\Upsilon_{1}^{(\nu)}\left[w_{B}, w_{B}\right](s)\right)\left(\xi_{1}, X_{2}\right)\right| \lesssim & \int_{0}^{s} \int_{0}^{\infty} e^{-\frac{3}{4} \nu(s-\tau) \xi_{1}^{2}} g\left(\frac{5}{4}(s-\tau), X_{2}+Y_{2}\right)\left(\frac{\nu^{\frac{1}{2}}\left\langle\xi_{1}\right\rangle^{2}}{(s-\tau)^{\frac{1}{2}}} \chi_{\left\{Y_{2} \leq \frac{\mu^{\prime}}{\nu^{\frac{1}{2}}}\right\}}\right. \\
& \left.+\frac{\nu\left\langle\xi_{1}\right\rangle}{\mu^{\prime 2}} \chi_{\left\{Y_{2} \geq \frac{\mu^{\prime}}{\nu^{\frac{1}{2}}}\right\}}\right)\left|\mathcal{F}\left(B^{(\nu)}\left(w_{B}, w_{B}\right)\right)\right|\left(\xi_{1}, Y_{2}\right) \mathrm{d} Y_{2} \mathrm{~d} \tau .
\end{aligned}
$$

Hence, as in the proof of (3.4), by using (4.64) and (4.65) we get

$$
\begin{gathered}
\left\|\Upsilon_{1}^{(\nu)}\left[w_{B}, w_{B}\right](s)\right\|_{X_{I B_{\nu}, 1}^{\left(\mu^{\prime}\right)}} \lesssim \int_{0}^{s} \frac{\nu^{\frac{1}{2}}}{(s-\tau)^{\frac{1}{2}}}\left\|\varphi_{B_{\nu}}^{\left(\mu^{\prime}, \frac{\rho}{s}\right)}\left\langle\xi_{1}\right\rangle^{2} \chi_{\left\{X_{2} \leq \frac{\mu^{\prime}}{\nu^{\frac{1}{2}}}\right\}} \mathcal{F}\left(B^{(\nu)}\left(w_{B}(\tau), w_{B}(\tau)\right)\right)\right\|_{L_{\xi_{1}}^{2} L_{X_{2}}^{1}} \mathrm{~d} \tau \\
\quad+\int_{0}^{s} \frac{\nu}{\mu^{\prime 2}}\left\|\varphi_{B_{\nu}}^{\left(\mu^{\prime}, \frac{\rho}{s}\right)}\left\langle\xi_{1}\right\rangle \chi_{\left\{X_{2} \geq \frac{\mu^{\prime}}{\nu^{\frac{1}{2}}}\right\}} \mathcal{F}\left(B^{(\nu)}\left(w_{B}(\tau), w_{B}(\tau)\right)\right)\right\|_{L_{\xi_{1}}^{2} L_{X_{2}}^{1}} \mathrm{~d} \tau \\
\lesssim \int_{0}^{s} \frac{\nu^{\frac{1}{2}}}{(s-\tau)^{\frac{1}{2}}\left(\mu-\mu^{\prime}\right)}\left\|w_{B}(\tau)\right\|_{X_{I B_{\nu}, 2}^{(\mu, \rho)}}^{2} \mathrm{~d} \tau+\int_{0}^{s} \frac{\nu}{\mu^{\prime 2}}\left\|w_{B}(\tau)\right\|_{\left.X_{I B_{\nu}, \frac{\rho}{s}}^{(\mu, 2}\right)}^{2} \mathrm{~d} \tau \lesssim \frac{\nu^{\frac{1}{2}} s^{\frac{1}{2}}}{16 d_{E}}\left\|w_{B}\right\|_{Z_{B_{\nu}}}^{2},
\end{gathered}
$$


if $0<\nu<d_{E}^{5}, \mu^{\prime}=d_{E} / 4, \mu=d_{E} / 2$, and $\rho=2^{-6}$. Then it is not difficult to get (4.63) from the arguments as above. The proof is complete.

\section{$5 \quad$ Proof of Theorem 1.1}

By Theorems 4.4, 4.10, 4.12, and 4.13, the solution $\omega=\omega^{(\nu)}$ of $\left(\mathrm{V}_{\nu}\right)$ is decomposed as $\omega^{(\nu)}=\omega_{E}+$ $R_{1 / \nu} w_{P}+R_{1 / \nu}\left(w_{B}^{(\nu)}-w_{P}+w_{I B}^{(\nu)}\right)+w_{I I}^{(\nu)}$, and it follows that

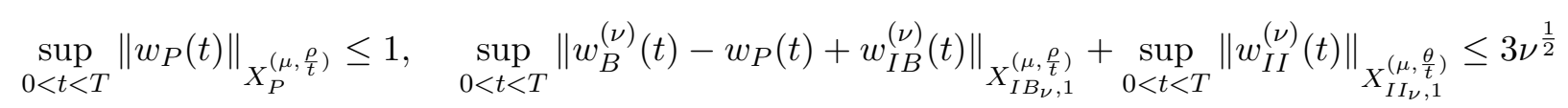

for $\mu=d_{E} / 8, \rho=2^{-7}, \theta=2^{-10}$, and $T=c d_{E}$ with $c>0$ depending only on $\|b\|_{Y_{E}}$. Since $u_{N S}^{(\nu)}=$ $J\left(\omega^{(\nu)}\right)$ and $u_{E}=J\left(\omega_{E}\right)$, Lemma 2.5 implies $\sup _{0<t<T}\left\|J\left(R_{1 / \nu} w_{P}\right)(t)\right\|_{L^{\infty}} \leq C$ and $\sup _{0<t<T} \| u_{N S}^{(\nu)}(t)-u_{E}(t)-$ $J\left(R_{1 / \nu} w_{P}\right)(t) \|_{L^{\infty}} \leq C \nu^{1 / 2}$ with a numerical constant $C>0$. Let $v_{P}=\left(v_{P, 1}, v_{P, 2}\right)$ be the velocity field defined in (2.4) - (2.5). It suffices to show

$$
\sup _{0<t<T}\left(\left\|\nu^{\frac{1}{2}} R_{\frac{1}{\nu}} v_{P, 1}(t)-J_{1}\left(R_{\frac{1}{\nu}} w_{P}\right)(t)\right\|_{L^{\infty}}+\nu^{\frac{1}{2}}\left\|v_{P, 2}(t)\right\|_{L^{\infty}}+\left\|J_{2}\left(R_{\frac{1}{\nu}} w_{P}\right)(t)\right\|_{L^{\infty}}\right) \leq C \nu^{\frac{1}{2}}
$$

We give the proof only for the first term of the left-had side of (5.1). The other terms are handled with similarly. Lemma 2.4 implies

$$
\begin{aligned}
\left|\mathcal{F}\left(v_{P, 1}(t)-\nu^{-\frac{1}{2}} R_{\nu} J_{1}\left(R_{\frac{1}{\nu}} w_{P}\right)(t)\right)\left(\xi_{1}, X_{2}\right)\right| & \leq \frac{1}{2} \int_{0}^{X_{2}} e^{-\nu^{\frac{1}{2}}\left|\xi_{1}\right|\left(X_{2}-Y_{2}\right)}\left(1-e^{-2 \nu^{\frac{1}{2}}\left|\xi_{1}\right| Y_{2}}\right)\left|\hat{w}_{P}\left(t, \xi_{1}, Y_{2}\right)\right| \mathrm{d} Y_{2} \\
& +\frac{1}{2} \int_{X_{2}}^{\infty}\left(2-e^{-\nu^{\frac{1}{2}}\left|\xi_{1}\right|\left|X_{2}-Y_{2}\right|}\left(1+e^{-2 \nu^{\frac{1}{2}}\left|\xi_{1}\right| Y_{2}}\right)\right)\left|\hat{w}_{P}\left(t, \xi_{1}, Y_{2}\right)\right| \mathrm{d} Y_{2} \\
& \leq C \nu^{\frac{1}{2}}\left\|\left\langle\xi_{1}\right\rangle Y_{2} \hat{w}_{P}\left(t, \xi_{1}, Y_{2}\right)\right\|_{L_{Y_{2}}^{1}}
\end{aligned}
$$

which leads to $\left\|\left\langle\xi_{1}\right\rangle \mathcal{F}\left(v_{P, 1}(t)-\nu^{-1 / 2} R_{\nu} J_{1}\left(R_{1 / \nu} w_{P}\right)(t)\right)\right\|_{L_{\xi_{1}}^{2} L_{X_{2}}^{\infty}} \leq C \nu^{1 / 2}\left\|w_{P}\right\|_{Z_{P}}$ for $0<t<T$. Hence (5.1) follows by using $\left\|s^{1 / 2} R_{1 / s} f\right\|_{L^{\infty}}=\|f\|_{L^{\infty}}$. This completes the proof.

\section{Open problem}

In the proof of Theorem 1.1 the condition (1.2) plays essential roles. If (1.2) is absent and the initial data is not analytic it is believed that the separation of the boundary layer immediately occurs in general and the vorticity behaves rather intricately, which is difficult to control. In particular, it is hard to expect that the vorticity keeps the simple form as in (1.1) for $0<t \leq \mathcal{O}(1)$. For general initial data the expansion like (1.1) is verified so far only for a time period $0<t \leq \mathcal{O}\left(\nu^{1 / 3}\right)$; [20]. It is not known whether the exponent $1 / 3$ can be improved or not. More importantly, it is not clear how to estimate the interaction between the vorticity generated near the boundary and the vorticity away from the boundary without the condition (1.2), which causes the lack of the effective bound of the vorticity even in the region $\Omega_{c}=\left\{x \in \mathbb{R}_{+}^{2} \mid x_{2} \geq c\right\}$ for a positive $c$. In view of (7.6), or if one reminds the trajectory flow determined by $u$, it is important to control the quantity $\|u\|_{L^{1}\left(0, t ; L^{\infty}\left(\Omega_{c}\right)\right)}$. But so far the uniform bound (with respect to the small viscosity) for this quantity is absent even if $L^{\infty}$ is replaced by $L^{p}$ for some $p>2$ when the initial data is taken from a Sobolev class. 


\section{Appendix}

\subsection{Young inequality in weighted function spaces}

Lemma 7.1 Assume that $1 \leq q \leq p \leq \infty$ and $0<\beta<1 / 4$. Then the following estimates hold.

$$
\begin{aligned}
& \left\|e^{\frac{\beta}{t} X_{2}^{2}} g(t-s) * f\left(X_{2}\right)\right\|_{L_{X_{2}}^{p}} \lesssim(1-4 \beta)^{-\frac{1}{2}\left(1+\frac{1}{p}-\frac{1}{q}\right)}(t-s)^{-\frac{1}{2}\left(\frac{1}{q}-\frac{1}{p}\right)}\left\|e^{\frac{\beta}{s} X_{2}^{2}} f\left(X_{2}\right)\right\|_{L_{X_{2}}^{q}}, \\
& \left\|e^{\frac{\beta}{t}\left(6 d_{E}-x_{2}\right)_{+}^{2}} g(t-s) * f\left(x_{2}\right)\right\|_{L_{x_{2}}^{p}} \lesssim(1-4 \beta)^{-\frac{1}{2}\left(1+\frac{1}{p}-\frac{1}{q}\right)}(t-s)^{-\frac{1}{2}\left(\frac{1}{q}-\frac{1}{p}\right)}\left\|e^{\frac{\beta}{s}\left(6 d_{E}-x_{2}\right)_{+}^{2}} f\left(x_{2}\right)\right\|_{L_{x_{2}}^{q}} .
\end{aligned}
$$

Proof. We give the proof only when $p<\infty$. Set $1 / r=1+1 / p-1 / q$. The Hölder inequality yields

$$
\begin{aligned}
e^{\frac{\beta}{t} X_{2}^{2}} \int_{\mathbb{R}} g\left(t-s, X_{2}-Y_{2}\right)\left|f\left(Y_{2}\right)\right| \mathrm{d} Y_{2} \leq\left(\int_{\mathbb{R}} e^{\frac{r \beta}{t} X_{2}^{2}-\frac{r \beta}{s} Y_{2}^{2}} g\left(t-s, X_{2}-Y_{2}\right)^{r} \mathrm{~d} Y_{2}\right)^{1-\frac{1}{q}} \\
\cdot\left(\int_{\mathbb{R}} e^{\frac{r \beta}{t} X_{2}^{2}-\frac{r \beta}{s} Y_{2}^{2}} g\left(t-s, X_{2}-Y_{2}\right)^{r}\left|e^{\frac{\beta}{s} Y_{2}^{2}} f\left(Y_{2}\right)\right|^{q} \mathrm{~d} Y_{2}\right)^{\frac{1}{p}}\left\|e^{\frac{\beta}{s} Y_{2}^{2}} f\right\|_{L_{Y_{2}}^{q}}^{1-\frac{q}{p}} .
\end{aligned}
$$

Then we use the equalities

$$
\begin{aligned}
& \frac{\beta X_{2}^{2}}{t}-\frac{\left|X_{2}-Y_{2}\right|^{2}}{4(t-s)}-\frac{\beta Y_{2}^{2}}{s}=-\frac{(1-4 \beta) t+4 \beta s}{4 t(t-s)}\left|X_{2}-\frac{t}{(1-4 \beta) t+4 \beta s} Y_{2}\right|^{2}-\frac{\beta(1-4 \beta)(t-s)}{s\{(1-4 \beta) t+4 \beta s\}} Y_{2}^{2}, \\
& \frac{\beta X_{2}^{2}}{t}-\frac{\left|X_{2}-Y_{2}\right|^{2}}{4(t-s)}-\frac{\beta Y_{2}^{2}}{s}=-\frac{4 \beta t+(1-4 \beta) s}{4(t-s) s}\left|Y_{2}-\frac{s}{4 \beta t+(1-4 \beta) s} X_{2}\right|^{2}-\frac{\beta(1-4 \beta)(t-s)}{t\{4 \beta t+(1-4 \beta) s\}} X_{2}^{2} .
\end{aligned}
$$

Hence (7.3) implies

$$
\begin{aligned}
& e^{\frac{\beta}{t} X_{2}^{2}} \int_{\mathbb{R}} g\left(t-s, X_{2}-Y_{2}\right)\left|f\left(Y_{2}\right)\right| \mathrm{d} Y_{2} \\
\lesssim & (1-4 \beta)^{-\frac{1}{2}\left(1-\frac{1}{q}\right)}(t-s)^{\frac{1}{2}\left(1-\frac{1}{q}\right)(1-r)}\left(\int_{\mathbb{R}} e^{\frac{r \beta}{t} X_{2}^{2}-\frac{r \beta}{s} Y_{2}^{2}} g\left(t-s, X_{2}-Y_{2}\right)^{r}\left|e^{\frac{\beta}{s} Y_{2}^{2}} f\left(Y_{2}\right)\right|^{q} \mathrm{~d} Y_{2}\right)^{\frac{1}{p}}\left\|e^{\frac{\beta}{s} Y_{2}^{2}} f\right\|_{L_{Y_{2}}^{q} \frac{q}{p}}^{1-},
\end{aligned}
$$

and (7.1) is easily obtained from this inequality. To prove (7.2) we observe that

$\left\|e^{\frac{\beta}{t}\left(6 d_{E}-x_{2}\right)^{2}} g(t-s) * f\left(x_{2}\right)\right\|_{L_{x_{2}}^{p}} \leq\left\|e^{\frac{\beta}{t}\left(6 d_{E}-x_{2}\right)^{2}} g(t-s) * f\left(x_{2}\right)\right\|_{L_{x_{2}}^{p}\left(\left\{x_{2} \leq 6 d_{E}\right\}\right)}+\left\|g(t-s) * f\left(x_{2}\right)\right\|_{L_{x_{2}}^{p}\left(\left\{x_{2} \geq 6 d_{E}\right\}\right)}$, and thus, it suffices to estimate $\left\|e^{\beta\left(6 d_{E}-x_{2}\right)^{2} / t} g(t-s) * f\left(x_{2}\right)\right\|_{L_{x_{2}}^{p}\left(\left\{x_{2} \leq 6 d_{E}\right\}\right)}$. Set $\tilde{x}_{2}=6 d_{E}-x_{2}$ and $\tilde{y}_{2}=6 d_{E}-y_{2}$. Then for $x_{2} \leq 6 d_{E}$ we have

$$
g(t-s) * f\left(x_{2}\right)=\int_{-\infty}^{6 d_{E}} g\left(t-s, x_{2}-y_{2}\right) f\left(y_{2}\right) \mathrm{d} y_{2}+\int_{6 d_{E}}^{\infty} g\left(t-s, x_{2}-y_{2}\right) f\left(y_{2}\right) \mathrm{d} y_{2}=\sum_{i=1}^{2} I_{i} .
$$

Since $g\left(t-s, x_{2}-y_{2}\right)=g\left(t-s, \tilde{x}_{2}-\tilde{y}_{2}\right)$, by arguing as in the proof of $(7.1), I_{1}$ is estimated as

$$
\left\|e^{\frac{\beta}{t} \tilde{x}_{2}^{2}} I_{1}\right\|_{L^{p}\left(\left\{x_{2} \leq 6 d_{E}\right\}\right)} \lesssim(1-4 \beta)^{-\frac{1}{2}\left(1+\frac{1}{p}-\frac{1}{q}\right)}(t-s)^{-\frac{1}{2}\left(\frac{1}{q}-\frac{1}{p}\right)}\left\|e^{\frac{\beta}{s}\left(6 d_{E}-x_{2}\right)_{+}^{2}} f\right\|_{L_{x_{2}}^{q}} .
$$

As for $I_{2}$, we have from the Hölder inequality and from $\tilde{x}_{2} \geq 0$ and $\tilde{y}_{2} \leq 0$ when $x_{2} \leq 6 d_{E}$ and $y_{2} \geq 6 d_{E}$,

$$
\begin{aligned}
e^{\frac{\beta}{t} \tilde{x}_{2}^{2}}\left|I_{2}\right| & \leq\left(\int_{6 d_{E}}^{\infty} e^{\frac{r \beta}{t} \tilde{x}_{2}^{2}} g\left(t-s, \tilde{x}_{2}-\tilde{y}_{2}\right)^{r} \mathrm{~d} y_{2}\right)^{1-\frac{1}{q}}\left(\int_{6 d_{E}}^{\infty} e^{\frac{r \beta}{t} \tilde{x}_{2}^{2}} g\left(t-s, \tilde{x}_{2}-\tilde{y}_{2}\right)^{r}\left|f\left(y_{2}\right)\right|^{q} \mathrm{~d} y_{2}\right)^{\frac{1}{p}}\|f\|_{L^{q}}^{1-\frac{q}{p}} \\
& \lesssim e^{-\frac{(1-4 \beta) t+4 \beta s}{4 t(t-s)} \tilde{x}_{2}^{2}}\left(\int_{6 d_{E}}^{\infty} g\left(t-s, \tilde{y}_{2}\right)^{r} \mathrm{~d} y_{2}\right)^{1-\frac{1}{q}}\left(\int_{6 d_{E}}^{\infty} g\left(t-s, \tilde{y}_{2}\right)^{r}\left|f\left(y_{2}\right)\right|^{q} \mathrm{~d} y_{2}\right)^{\frac{1}{p}}\|f\|_{L^{q}}^{1-\frac{q}{p}} \\
& \lesssim e^{-\frac{(1-4 \beta) t+4 \beta s}{4 t(t-s)} \tilde{x}_{2}^{2}}(t-s)^{-\frac{1}{2 q}}\|f\|_{L^{q}} .
\end{aligned}
$$

Hence we have $\left\|e^{\beta \tilde{x}_{2}^{2} / t} I_{2}\right\|_{L^{p}\left(\left\{x_{2} \leq 6 d_{E}\right\}\right)} \lesssim(1-4 \beta)^{-1 /(2 p)}(t-s)^{-1 / 2(1 / q-1 / p)}\|f\|_{L^{q}}$. The proof is complete. 


\subsection{Kernel for the heat-transport equations}

Lemma 7.2 Let $P_{u}^{(\nu)}(t, s)$ be the evolution operator associated with $(H T)$, and let $P_{u}^{(\nu)}(t, x ; s, y)$ be the kernel of $P_{u}^{(\nu)}(t, s)$. Then for $1 \leq q \leq p \leq \infty$,

$$
\left\|P_{u}^{(\nu)}(t, s) f\right\|_{L^{p}} \lesssim(\nu(t-s))^{-\frac{1}{q}+\frac{1}{p}}\|f\|_{L^{q}} \quad 0 \leq s<t<\infty,
$$

and if $F=\left(F_{1}, F_{2}\right)$ satisfies $F_{2}=0$ on $\partial \mathbb{R}_{+}^{2}$ then

$$
\left\|\int_{0}^{t} P_{u}^{(\nu)}(t, s) \nabla \cdot F \mathrm{~d} s\right\|_{L^{2}} \lesssim \nu^{-\frac{1}{2}}\|F\|_{L^{2}\left(0, t ; L^{2}\right)} \quad 0 \leq s<t<\infty .
$$

Moreover, we have

$0 \leq P_{u}^{(\nu)}(t, x ; s, y) \leq \frac{1}{2 \pi \nu(t-s)} \exp \left(-\frac{1}{4 \nu(t-s)}\left(|x-y|-\int_{s}^{t}\|u(\tau)\|_{L^{\infty}} \mathrm{d} \tau\right)_{+}^{2}\right), \quad(\alpha)_{+}=\max \{\alpha, 0\}$.

Proof. The estimate (7.5) is a simple application of the energy calculations based on the integration by parts, so we omit the details here. Let $H$ be the solution of (HT). Then by setting $\tilde{H}(t, x)=H(t / \nu, x)$ if $x_{2} \geq 0$ and $\tilde{H}(t, x)=H\left(t / \nu, x^{*}\right)$ if $x_{2}<0$ with $x^{*}=\left(x_{1},-x_{2}\right)$ the problem (HT) is reduced to the equation in the whole plane

$$
\left\{\begin{aligned}
\partial_{t} \tilde{H}-\Delta \tilde{H}+\tilde{u} \cdot \nabla \tilde{H} & =\tilde{f} & t>0, & x \in \mathbb{R}^{2} \\
\left.\tilde{H}\right|_{t=0} & =0 & & x \in \mathbb{R}^{2} .
\end{aligned}\right.
$$

Here $\tilde{u}=\left(\tilde{u}_{1}, \tilde{u}_{2}\right)$ is defined by

$$
\tilde{u}_{1}(t, x)=\left\{\begin{array}{ll}
\nu^{-1} u_{1}(t / \nu, x) & \text { if } x_{2} \geq 0 \\
\nu^{-1} u_{1}\left(t / \nu, x^{*}\right) & \text { if } x_{2}<0
\end{array} \quad \tilde{u}_{2}(t, x)= \begin{cases}\nu^{-1} u_{2}(t / \nu, x) & \text { if } x_{2} \geq 0 \\
-\nu^{-1} u_{2}\left(t / \nu, x^{*}\right) & \text { if } x_{2}<0\end{cases}\right.
$$

and $\tilde{f}(t, x)=\nu^{-1} f(t / \nu, x)$ if $x_{2} \geq 0$ and $\tilde{f}(t, x)=\nu^{-1} f\left(t / \nu, x^{*}\right)$ if $x_{2}<0$. Clearly $\tilde{u}$ satisfies div $\tilde{u}=0$ in $\mathbb{R}^{2}$ since $u_{2}=0$ on $\partial \mathbb{R}_{+}^{2}$. Let $P_{\tilde{u}}(t, s)$ be the evolution operator associated with (7.7). Then we have

$$
\tilde{H}(\nu t)=\int_{0}^{\nu t} P_{\tilde{u}}(\nu t, \nu \tau) \nu \tilde{f}(\nu \tau) \mathrm{d} \tau
$$

To show (7.4) we take $\nu \tilde{f}(\nu t, x)=\nu \tilde{f}(x) \delta_{\{t=\nu s\}}$, where $\tilde{f}$ is the extension of the time-independent function $f$ in $\mathbb{R}_{+}^{2}$ by the above reflection. Then we have $\tilde{H}(\nu t)=P_{\tilde{u}}(\nu t, \nu s) \nu \tilde{f}$ and the $L^{p}-L^{q}$ estimate in $[5$, Theorem 1] implies $\|\tilde{H}(\nu t)\|_{L^{p}\left(\mathbb{R}^{2}\right)} \lesssim(\nu(t-s))^{-1 / q+1 / p}\|\nu \tilde{f}\|_{L^{q}\left(\mathbb{R}^{2}\right)} \lesssim(\nu(t-s))^{-1 / q+1 / p}\|f\|_{L^{q}\left(\mathbb{R}_{+}^{2}\right)}$. The estimate (7.4) then follows from the relation $H(t)=\tilde{H}(\nu t) \chi_{\left\{x_{2} \geq 0\right\}}$. Let $P_{\tilde{u}}(t, x ; s, y)$ be the kernel of $P_{\tilde{u}}(t, s)$. It is well-known that $P_{\tilde{u}}(t, x ; s, y)$ is positive. From $(7.9)$ we have

$$
\tilde{H}(\nu t, x)=\int_{0}^{\nu t} \int_{\mathbb{R}^{2}} P_{\tilde{u}}(\nu t, x ; \nu \tau, z) \nu \tilde{f}(\nu \tau, z) \mathrm{d} z \mathrm{~d} \tau,
$$

which yields, by taking $f(t, x)=\delta_{\{x=y\}} \delta_{\{t=s\}}$,

$$
P_{u}^{(\nu)}(t, x ; s, y)=P_{\tilde{u}}(\nu t, x ; \nu s, y)+P_{\tilde{u}}\left(\nu t, x ; \nu s, y^{*}\right)
$$

for $x, y \in \mathbb{R}_{+}^{2}$. Now we recall the pointwise estimate by [5, Theorem 3] as follows.

$$
P_{\tilde{u}}(\nu t, x ; \nu s, y) \leq \frac{1}{4 \pi \nu(t-s)} \exp \left(-\frac{1}{\nu(t-s)}\left(|x-y|-\int_{\nu s}^{\nu t}\|\tilde{u}(\tau)\|_{L^{\infty}} \mathrm{d} \tau\right)_{+}^{2}\right) .
$$

Hence (7.6) holds by (7.10) and (7.11). The proof is complete. 


\subsection{Lemma for Theorem 4.12}

Lemma 7.3 Assume that $0<s<t<T_{0}, d_{E} / 8 \leq \mu-\mu^{\prime}<\mu \leq d_{E}, 2^{-7}<\rho-\rho^{\prime}<\rho \leq 2^{-4}, 0<\nu \leq d_{E}^{3}$, and $j=1,2$. Then the following estimates hold.

$$
\begin{aligned}
& \left\|e^{(t-s) A} B^{(0)}\left(R_{0} \omega_{E}, h\right)+\Phi_{B, 1}^{(\nu)}\left[R_{\nu} \omega_{E}, h\right](t-s)\right\|_{X_{B_{\nu}}^{\left(\mu^{\prime}, \frac{\rho^{\prime}}{t}\right)}} \lesssim \frac{\nu^{\frac{1}{2} t}}{d_{E}(t-s)^{\frac{1}{2}}}\left\|\omega_{E}\right\|_{Z_{E}}\|h\|_{X_{P}^{\left(\mu, \frac{\rho}{s}\right)}},
\end{aligned}
$$

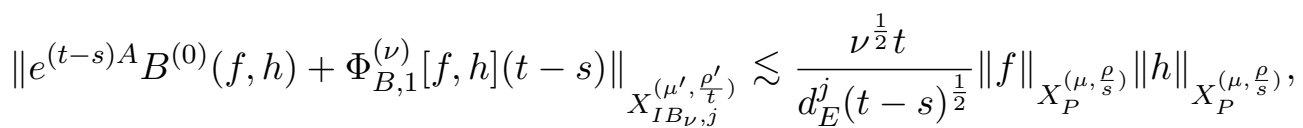

$$
\begin{aligned}
& \left\|e^{(t-s) A}\left(N^{(0)}\left(R_{0} \omega_{E}, h\right) \mathcal{H}_{\left\{X_{2}=0\right\}}^{1}\right)+\Phi_{B, 2}^{(\nu)}\left[R_{\nu} \omega_{E}, h\right](t-s)\right\|_{X_{I B_{\nu}, j}^{\left(\mu^{\prime}, \rho^{\prime}\right)}} \lesssim \frac{\nu^{\frac{1}{2}} t^{\frac{1}{2}}}{d_{E}^{j}}\left\|\omega_{E}\right\|_{Z_{E}}\|h\|_{X_{P}^{\left(\mu, \frac{\rho}{s}\right)}},
\end{aligned}
$$

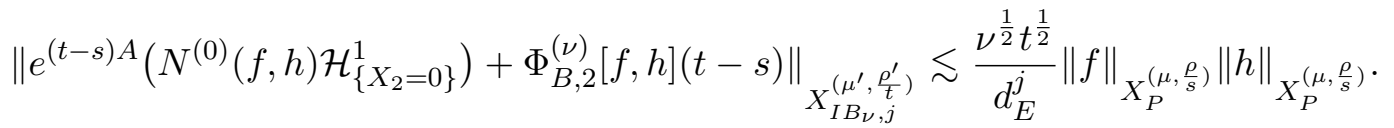

Proof. We give the proof only for (7.12) and (7.13), the other two are estimated in the same manner. We recall that $\omega_{E}=\omega_{E}(s), 0<s<T_{0}$, satisfies supp $R_{\nu} \hat{\omega}_{E} \subset\left\{X_{2} \geq 32 d_{E} \nu^{-1 / 2}\right\}$. The arguments as in the proof of Lemma 3.3 lead to the estimate for the case $Y_{2} \leq \nu^{-1 / 2} \mu$ such as

$$
\begin{aligned}
& \left|\mathcal{F}\left(B_{1}^{(0)}\left(R_{0} \omega_{E}, h\right)-B_{1}^{(\nu)}\left(R_{\nu} \omega_{E}, h\right)\right)\left(\xi_{1}, Y_{2}\right)\right| \\
\lesssim & \int_{\mathbb{R}}\left\|\chi_{\left\{Z_{2} \geq \frac{32 d_{E}}{\nu^{1 / 2}}\right\}} e^{-\nu^{\frac{1}{2}}\left|\eta_{1}\right|\left(\cdot-Y_{2}\right)}\left(2 e^{-\nu^{\frac{1}{2}}\left|\eta_{1}\right| Y_{2}}-1-e^{-2 \nu^{\frac{1}{2}}\left|\eta_{1}\right| Y_{2}}\right) R_{\nu} \hat{\omega}_{E}\right\|_{L_{Z_{2}}^{1}}\left|\left(\xi_{1}-\eta_{1}\right) \hat{h}\left(\xi_{1}-\eta_{1}, Y_{2}\right)\right| \mathrm{d} \eta_{1}, \\
& \left|\mathcal{F}\left(B_{2}^{(0)}\left(R_{0} \omega_{E}, h\right)-B_{2}^{(\nu)}\left(R_{\nu} \omega_{E}, h\right)\right)\left(\xi_{1}, Y_{2}\right)\right| \\
\lesssim & \int_{\mathbb{R}}\left\|\chi_{\left\{Z_{2} \geq \frac{32 d_{E}}{\nu^{1 / 2}}\right\}} \frac{e^{-\nu^{\frac{1}{2}}\left|\eta_{1}\right|\left(\cdot-Y_{2}\right)}}{\nu^{\frac{1}{2}}}\left(1-e^{-2 \nu^{\frac{1}{2}}\left|\eta_{1}\right| Y_{2}}-2 \nu^{\frac{1}{2}}\left|\eta_{1}\right| Y_{2} e^{-\nu^{\frac{1}{2}}\left|\eta_{1}\right| Y_{2}}\right) R_{\nu} \hat{\omega}_{E}\right\|_{L_{Z_{2}}^{1}}\left|\partial_{Y_{2}} \hat{h}\left(\xi_{1}-\eta_{1}, Y_{2}\right)\right| \mathrm{d} \eta_{1} .
\end{aligned}
$$

Thus, if $Y_{2} \leq \nu^{-1 / 2} \mu$ then $I\left(s, \xi_{1}, Y_{2}\right):=\mathcal{F}\left(B^{(0)}\left(R_{0} \omega_{E}, h\right)-B^{(\nu)}\left(R_{\nu} \omega_{E}, h\right)\right)\left(\xi_{1}, Y_{2}\right)$ satisfies

$$
\begin{aligned}
\left|I\left(s, \xi_{1}, Y_{2}\right)\right| \lesssim & \int_{\mathbb{R}} \frac{\nu^{\frac{1}{2}} Y_{2} e^{-\frac{\mu^{\prime}}{4}\left|\xi_{1}\right|-\frac{\rho}{s} Y_{2}^{2}}}{\left(\left|\eta_{1}\right|+\left|\xi_{1}-\eta_{1}\right|\right)\left(\mu-\mu^{\prime}\right)}\left\|\eta_{1}^{\frac{1}{2}} \hat{\omega}_{E}\right\|_{L_{x_{2}}^{2}}\left|\left(\xi_{1}-\eta_{1}\right) \hat{h}_{\left(\mu, \frac{\rho}{s}\right)}\left(\xi_{1}-\eta_{1}, Y_{2}\right)\right| \mathrm{d} \eta_{1} \\
& +\int_{\mathbb{R}} \frac{\nu^{\frac{1}{2}} Y_{2} e^{-\frac{\mu^{\prime}}{4}\left|\xi_{1}\right|-\frac{\rho}{s} Y_{2}^{2}}}{\left(\left|\eta_{1}\right|+\left|\xi_{1}-\eta_{1}\right|\right)\left(\mu-\mu^{\prime}\right)}\left\|\eta_{1}^{\frac{3}{2}} \hat{\omega}_{E}\right\|_{L_{x_{2}}^{2}} Y_{2}\left|\left(\partial_{Y_{2}} \hat{h}\right)_{\left(\mu, \frac{\rho}{s}\right)}\left(\xi_{1}-\eta_{1}, Y_{2}\right)\right| \mathrm{d} \eta_{1} .
\end{aligned}
$$

Here we have set $\hat{f}_{(\mu, \rho / s)}=\varphi_{P}^{(\mu, \rho / s)} \hat{f}$. Thus we get for $k=0,1$,

$$
\left\|\varphi_{B_{\nu}}^{\left(\mu^{\prime}, \frac{\rho^{\prime}}{s}\right)}\left\langle\xi_{1}\right\rangle^{2} Y_{2}^{\frac{k}{2}} \chi_{\left\{Y_{2} \leq \frac{\mu}{\nu^{1 / 2}}\right\}} I(s)\right\|_{L_{\xi_{1}}^{2} L_{Y_{2}}^{1+k}} \lesssim \frac{\nu^{\frac{1}{2}} s^{\frac{1}{2}}}{\left(\mu-\mu^{\prime}\right)\left(\rho-\rho^{\prime}\right)^{\frac{1}{2}}}\left\|\omega_{E}\right\|_{Z_{E}}\|h\|_{X_{P}^{\left(\mu, \frac{\rho}{s}\right)}} .
$$

If $Y_{2} \geq \nu^{-1 / 2} \mu$ then we use the estimate

$$
\begin{aligned}
\left|\mathcal{F}\left(B^{(0)}\left(R_{0} \omega_{E}, h\right)\right)\left(\xi_{1}, Y_{2}\right)\right| & \lesssim \int_{\mathbb{R}}\left\|\chi_{\left\{Z_{2} \geq \frac{32 d_{E}}{\nu^{1 / 2}}\right\}} e^{-\nu^{\frac{1}{2}}\left|\eta_{1} \| \cdot-Y_{2}\right|} R_{\nu} \hat{\omega}_{E}\right\|_{L_{Z_{2}}^{1}}\left|\left(\xi_{1}-\eta_{1}\right) \hat{h}\left(\xi_{1}-\eta_{1}, Y_{2}\right)\right| \mathrm{d} \eta_{1} \\
& \lesssim \int_{\mathbb{R}} \frac{e^{-\frac{\mu^{\prime}}{4}\left|\xi_{1}-\eta_{1}\right|-\frac{\rho}{s} Y_{2}^{2}}}{\mu-\mu^{\prime}}\left\|\hat{\omega}_{E}\right\|_{L_{x_{2}}^{1}}\left|\hat{h}_{\left(\mu, \frac{\rho}{s}\right)}\left(\xi_{1}-\eta_{1}, Y_{2}\right)\right| \mathrm{d} \eta_{1},
\end{aligned}
$$


and

$$
\left|\mathcal{F}\left(B^{(\nu)}\left(R_{\nu} \omega_{E}, h\right)\right)\left(\xi_{1}, Y_{2}\right)\right| \lesssim \int_{\mathbb{R}} \frac{e^{-\frac{\mu^{\prime}}{4}\left|\xi_{1}-\eta_{1}\right|-\frac{\rho}{s} Y_{2}^{2}}}{\left(1+\left|\xi_{1}-\eta_{1}\right|\right)\left(\mu-\mu^{\prime}\right)}\left\|\eta_{1}^{\frac{1}{2}} \hat{\omega}_{E}\right\|_{L_{x_{2}}^{2}} Y_{2}\left|\left(\partial_{Y_{2}} \hat{h}\right)_{\left(\mu, \frac{\rho}{s}\right)}\left(\xi_{1}-\eta_{1}, Y_{2}\right)\right| \mathrm{d} \eta_{1},
$$

which yields for $k=0,1$,

$$
\left\|\varphi_{B_{\nu}}^{\left(\mu^{\prime}, \frac{\rho^{\prime}}{s}\right)}\left\langle\xi_{1}\right\rangle^{2} Y_{2}^{\frac{k}{2}} \chi_{\left\{Y_{2} \geq \frac{\mu}{\nu^{1 / 2}}\right\}} I(s)\right\|_{L_{\xi_{1}}^{2} L_{Y_{2}}^{1+k}} \lesssim \frac{\nu s}{\mu^{2}\left(\mu-\mu^{\prime}\right)\left(\rho-\rho^{\prime}\right)}\left\|\omega_{E}\right\|_{Z_{E}}\|h\|_{X_{P}^{\left(\mu, \frac{\rho}{s}\right)}} .
$$

On the other hand, from (3.21) and (3.22) it is not difficult to show

$$
\left\|\varphi_{P}^{\left(\mu^{\prime}, \frac{\rho^{\prime}}{s}\right)}\left\langle\xi_{1}\right\rangle^{4} Y_{2}^{\frac{k}{2}} \mathcal{F}\left(B^{(0)}\left(R_{0} \omega_{E}, h\right)\right)\right\|_{L_{\xi_{1}}^{2} L_{Y_{2}}^{1+k}} \lesssim \frac{1}{\left(\mu-\mu^{\prime}\right)^{3}}\left\|\omega_{E}\right\|_{Z_{E}}\|h\|_{X_{P}^{\left(\mu, \frac{\rho}{s}\right)}} .
$$

Recalling the notation $g\left(t, X_{2}, Y_{2}\right)=g\left(t, X_{2}-Y_{2}\right)+g\left(t, X_{2}+Y_{2}\right)$, we decompose $e^{(t-s) A} B^{(0)}\left(R_{0} \omega_{E}, h\right)+$ $\Phi_{B, 1}^{(\nu)}\left[R_{\nu} \omega_{E}, h\right](t-s)$ into $I I_{1}(t, s)$ and $I I_{2}(t, s)$, where

$$
\begin{aligned}
& \mathcal{F}\left(I I_{1}\right)\left(t, s, \xi_{1}, X_{2}\right)=\int_{0}^{\infty} e^{-\nu(t-s) \xi_{1}^{2}} g\left(t-s, X_{2}, Y_{2}\right) I\left(s, \xi_{1}, Y_{2}\right) \mathrm{d} Y_{2}, \\
& \mathcal{F}\left(I I_{2}\right)\left(t, s, \xi_{1}, X_{2}\right)=\int_{0}^{\infty}\left(1-e^{-\nu(t-s) \xi_{1}^{2}}\right) g\left(t-s, X_{2}, Y_{2}\right) \mathcal{F}\left(B^{(0)}\left(R_{0} \omega_{E}, h\right)\right)\left(\xi_{1}, Y_{2}\right) \mathrm{d} Y_{2} .
\end{aligned}
$$

The estimates (3.4) and (3.5) yield

$$
\begin{aligned}
\left\|I I_{1}(t, s)\right\|_{\left.X_{B_{\nu}}^{\left(\mu^{\prime}\right.} \frac{\rho^{\prime}}{t}\right)} & \lesssim\left(1+\frac{s^{\frac{1}{2}}}{(t-s)^{\frac{1}{2}}\left(\rho-\rho^{\prime}\right)^{\frac{1}{2}}}\right) \sum_{k=0,1}\left\|\varphi_{B_{\nu}}^{\left(\mu^{\prime}, \frac{\rho^{\prime}}{s}\right)}\left\langle\xi_{1}\right\rangle^{2} X_{2}^{\frac{k}{2}} I(s)\right\|_{L_{\xi_{1}}^{2} L_{X_{2}}^{1+k}} \\
& \lesssim \frac{\nu^{\frac{1}{2}} s^{\frac{1}{2}}}{d_{E}}\left(1+\frac{\nu^{\frac{1}{2}} s^{\frac{1}{2}}}{\mu^{2}}\right)\left(1+\frac{s^{\frac{1}{2}}}{(t-s)^{\frac{1}{2}}}\right)\left\|\omega_{E}\right\|_{Z_{E}}\|h\|_{X_{P}^{\left(\mu, \frac{\rho}{s}\right)}} .
\end{aligned}
$$

Here we have used (7.16), (7.17), $\mu-\mu^{\prime}>d_{E} / 8$, and $\rho-\rho^{\prime}>2^{-7}$. On the other hand, by trivial modifications of the proofs for (3.4) and (3.5), we can derive the estimate

$$
\left\|e^{(t-s) A} f\right\|_{X_{P}^{\left(\mu^{\prime}, \rho_{t}^{\prime}\right)}} \lesssim\left(1+\frac{s^{\frac{1}{2}}}{(t-s)^{\frac{1}{2}}\left(\rho-\rho^{\prime}\right)^{\frac{1}{2}}}\right) \sum_{k=0,1}\left\|\varphi_{P}^{\left(\mu^{\prime}, \frac{\rho^{\prime}}{s}\right)}\left\langle\xi_{1}\right\rangle^{2} X_{2}^{\frac{k}{2}} \hat{f}\right\|_{L_{\xi_{1}}^{2} L_{X_{2}}^{1+k}}
$$

Hence, from $\left|1-e^{-\nu(t-s) \xi_{1}^{2}}\right| \leq C \nu(t-s) \xi_{1}^{2}$ and by using (7.18) we have

$$
\begin{aligned}
\left\|I I_{2}(t, s)\right\|_{X_{P}^{\left(\mu^{\prime}, \frac{\rho^{\prime}}{t}\right)}} & \lesssim \nu(t-s)\left(1+\frac{s^{\frac{1}{2}}}{(t-s)^{\frac{1}{2}}\left(\rho-\rho^{\prime}\right)^{\frac{1}{2}}}\right) \sum_{k=0,1}\left\|\xi_{1}^{2} \varphi_{P}^{\left(\mu^{\prime}, \frac{\rho^{\prime}}{s}\right)}\left\langle\xi_{1}\right\rangle^{2} X_{2}^{\frac{k}{2}} \mathcal{F}\left(B^{(0)}\left(R_{0} \omega_{E}, h\right)\right)\right\|_{L_{\xi_{1}}^{2} L_{X_{2}}^{1+k}} \\
& \lesssim \frac{\nu(t-s)}{d_{E}^{3}}\left(1+\frac{s^{\frac{1}{2}}}{(t-s)^{\frac{1}{2}}}\right)\left\|\omega_{E}\right\|_{Z_{E}}\|h\|_{X_{P}^{\left(\mu, \frac{\rho}{s}\right)}}
\end{aligned}
$$

Hence (7.12) holds from (7.19), (7.20), and the assumptions on the parameters. To prove (7.12) we see from (4.52) that

$$
\begin{aligned}
\left|\mathcal{F}\left(B^{(0)}(f, h)-B^{(\nu)}(f, h)\right)\left(\xi_{1}, Y_{2}\right)\right| \lesssim & \nu^{\frac{1}{2}} \int_{\mathbb{R}}\left\|\eta_{1} Z_{2} \hat{f}\right\|_{L_{Z_{2}}^{1}}\left|\left(\xi_{1}-\eta_{1}\right) \hat{h}\left(\xi_{1}-\eta_{1}, Y_{2}\right)\right| \mathrm{d} \eta_{1} \\
& +\nu^{\frac{1}{2}} \int_{\mathbb{R}}\left\|\eta_{1}^{2} Z_{2} \hat{f}\right\|_{L_{Z_{2}}^{1}} Y_{2}\left|\partial_{Y_{2}} \hat{h}\left(\xi_{1}-\eta_{1}, Y_{2}\right)\right| \mathrm{d} \eta_{1} .
\end{aligned}
$$


Hence for $I^{\prime}\left(\xi_{1}, Y_{2}\right)=\mathcal{F}\left(B^{(0)}(f, h)-B^{(\nu)}(f, h)\right)\left(\xi_{1}, Y_{2}\right)$ we have

$$
\left\|\varphi_{B_{\nu}}^{\left(\mu^{\prime}, \frac{\rho^{\prime}}{s}\right)}\left\langle\xi_{1}\right\rangle^{j} Y_{2}^{\frac{k}{2}} I^{\prime}\right\|_{L_{\xi_{1}}^{2} L_{Y_{2}}^{1+k}} \lesssim \frac{\nu^{\frac{1}{2}} s^{\frac{1}{2}}}{\left(\mu-\mu^{\prime}\right)^{j}\left(\rho-\rho^{\prime}\right)^{\frac{1}{2}}}\|f\|_{X_{P}^{\left(\mu, \frac{\rho}{s}\right)}}\|h\|_{X_{P}^{\left(\mu, \frac{\rho}{s}\right)}}, \quad j=1,2 .
$$

On the other hand, from the same arguments as in the proof of (3.21) and (3.22) one can derive

$$
\left\|\varphi_{P}^{\left(\mu^{\prime}, \frac{\rho^{\prime}}{s}\right)}\left\langle\xi_{1}\right\rangle^{2+j} Y_{2}^{\frac{k}{2}} \mathcal{F}\left(B^{(0)}(f, h)\right)\right\|_{L_{\xi_{1}}^{2} L_{Y_{2}}^{1+k}} \lesssim \frac{1}{\left(\mu-\mu^{\prime}\right)^{3}}\|f\|_{X_{P}^{\left(\mu, \frac{\rho}{s}\right)}}\|h\|_{X_{P}^{\left(\mu, \frac{\rho}{s}\right)}} .
$$

We decompose $e^{(t-s) A} B^{(0)}(f, h)+\Phi_{B, 1}^{(\nu)}[f, h](t-s)$ into $I I_{1}^{\prime}(t-s)$ and $I I_{2}^{\prime}(t-s)$, where

$$
\begin{aligned}
& \mathcal{F}\left(I I_{1}^{\prime}\right)\left(t-s, \xi_{1}, X_{2}\right)=\int_{0}^{\infty} e^{-\nu(t-s) \xi_{1}^{2}} g\left(t-s, X_{2}, Y_{2}\right) I^{\prime}\left(s, \xi_{1}, Y_{2}\right) \mathrm{d} Y_{2}, \\
& \mathcal{F}\left(I I_{2}^{\prime}\right)\left(t-s, \xi_{1}, X_{2}\right)=\int_{0}^{\infty}\left(1-e^{-\nu(t-s) \xi_{1}^{2}}\right) g\left(t-s, X_{2}, Y_{2}\right) \mathcal{F}\left(B^{(0)}(f, h)\right)\left(\xi_{1}, Y_{2}\right) \mathrm{d} Y_{2} .
\end{aligned}
$$

Then from (3.4), (3.5), (7.21), and (7.22), it is not difficult to see

$$
\begin{aligned}
&\left\|I I_{1}^{\prime}(t-s)\right\|_{X_{I B_{\nu}, j}^{\left(\mu^{\prime} \rho^{\prime}\right)}} \lesssim\left(1+\frac{s^{\frac{1}{2}}}{(t-s)^{\frac{1}{2}}\left(\rho-\rho^{\prime}\right)^{\frac{1}{2}}}\right) \frac{\nu^{\frac{1}{2}} s^{\frac{1}{2}}}{\left(\mu-\mu^{\prime}\right)^{j}\left(\rho-\rho^{\prime}\right)^{\frac{1}{2}}}\|f\|_{X_{P}^{\left(\mu, \frac{\rho}{s}\right)}\|h\|_{X_{P}^{\left(\mu, \frac{\rho}{s}\right)}},} \\
&\left\|I I_{2}^{\prime}(t-s)\right\|_{X_{P}^{\left(\mu^{\prime}, \frac{\rho^{\prime}}{t}\right)}} \lesssim\left(1+\frac{s^{\frac{1}{2}}}{(t-s)^{\frac{1}{2}}\left(\rho-\rho^{\prime}\right)^{\frac{1}{2}}} \frac{\nu(t-s)}{\left(\mu-\mu^{\prime}\right)^{3}}\|f\|_{X_{P}^{\left(\mu, \frac{\rho}{s}\right)}\|h\|_{X_{P}^{\left(\mu, \frac{\rho}{s}\right)} .}}\right.
\end{aligned}
$$

Here $j=1,2$. Thus (7.13) follows from (7.23), (7.24), and the assumptions on the parameters. By arguing as above, The estimates (7.14) and (7.15) are proved from the equality

$N^{(0)}(f, h)-N^{(\nu)}(f, h)=\int_{0}^{\infty} e^{-\nu^{\frac{1}{2}}\left|\xi_{1}\right| Y_{2}}\left(B^{(0)}(f, h)-B^{(\nu)}(f, h)\right) \mathrm{d} Y_{2}+\int_{0}^{\infty}\left(1-e^{-\nu^{\frac{1}{2}}\left|\xi_{1}\right| Y_{2}}\right) B^{(0)}(f, h) \mathrm{d} Y_{2}$.

The details are omitted. The proof is complete.

Acknowledgment. The author is grateful very much to Professor Yoshikazu Giga and Professor Takashi Sakajo for their helpful comments on this work. The author is partially supported by Grant-in-Aid for Young Scientists (B) 22740090.

\section{References}

[1] C. R. Anderson, Vorticity boundary conditions and boundary vorticity generation for twodimensional viscous incompressible flows. J. Comput. Phys. 80 (1989) 72-97.

[2] K. Asano, Zero viscosity limit of incompressible Navier-Stokes equations II, Surikaisekikenkyusho Kokyuroku, Mathematical analysis of fluid and plasma dynamics I (Kyoto, 1986) 656 (1988) 105-128.

[3] C. Bardos, Existence et unicité de la solution de l'équation d'Euler en dimension deux. J. Math. Anal. Appl. 40 (1972) 769-790.

[4] J. Bona, J. Wu, The zero-viscosity limit of the 2D Navier-Stokes equations. Stud. Appl. Math. 109 (2002) 265-278. 
[5] E. A. Carlen, M. Loss, Optimal smoothing and decay estimates for viscously damped conservation laws, with applications to the 2-D Navier-Stokes equation. Duke Math. J. 81 (1995) 135-157.

[6] J.-Y. Chemin, A remark on the inviscid limit for two-dimensional incompressible fluids. Comm. Partial Differential Equations 21 (1996) 1771-1779.

[7] M.-H. Giga, Y. Giga, J. Saal, Nonlinear partial differential equations: Asymptotic behavior of solutions and self-similar solutions, Birkhäuser, Boston-Basel-Berlin, 2010.

[8] P. Constantin, J. Wu, Inviscid limit for vortex patches. Nonlinearity 8(5) (1995) 735-742.

[9] P. Constantin, J. Wu, The inviscid limit for non-smooth vorticity. Indiana Univ. Math. J. 45(1) (1996) 67-81.

[10] E. Grenier, On the nonlinear instability of Euler and Prandtl equations. Comm. Pure Appl. Math. Vol. LIII (2000) 1067-1091.

[11] T. Kano, T. Nishida, Sur les ondes de surface de l'eau avec une justification mathématique des équations des ondes en eau peu profonde. J. Math. Kyoto Univ. 19 (1979) 335-370.

[12] T. Kato, On classical solutions of the two-dimensional nonstationary Euler equation. Arch. Ration. Mech. Anal. 25 (1967) 188-200.

[13] T. Kato, Remarks on zero viscosity limit for nonstationary Navier-Stokes flows with boundary. Seminar on nonlinear partial differential equations (Berkeley, 1983). Math. Sci. Res. Inst. Publ. 2 Springer, New York (1984) 85-98.

[14] J. P. Kelliher, On Kato's conditions for vanishing viscosity. Indiana Univ. Math. J. 56 (2007) 1711-1721.

[15] J. P. Kelliher, On the vanishing viscosity limit in a disk. Math. Ann. 343 (2009) 701-726.

[16] W. Kramer, H. J. H. Clercx, G. J. F. van Heijst, Vorticity dynamics of a dipole colliding with a no-slip wall. Phys. Fluids 19 (2007) 126603.

[17] M. C. Lombardo, M. Cannone, M. Sammartino, Well-posedness of the boundary layer equations. Siam J. Math. Anal. 35 (2003) 987-1004.

[18] M. C. Lopes Filho, A. Mazzucato, H. Nussenzveig Lopes, Vanishing viscosity limit for incompressible flow inside a rotating circle. Physica D. 237 (2008) 1324-1333.

[19] M. C. Lopes Filho, A. Mazzucato, H. Nussenzveig Lopes, M. Taylor, Vanishing viscosity limits and boundary layers for circularly symmetric 2D flows. Bull. Brazilian Math. Soc. 39 (2008) 471-513.

[20] Y. Maekawa, Solution formula for the vorticity equations in the half plane with application to high vorticity creation at zero viscosity limit. Hokkaido university preprint series \# 992; available at http://eprints3.math.sci.hokudai.ac.jp/2173/

[21] A. J. Majda, A. L. Bertozzi, Vorticity and incompressible flows, Cambridge University Press, Cambridge, 2002.

[22] N. Masmoudi, Remarks about the inviscid limit of the Navier-Stokes system. Commun. Math. Phys. 270 (2007) 777-788. 
[23] S. Matsui, Example of zero viscosity limit for two-dimensional nonstationary Navier-Stokes flows with boundary. Japan. J. Indust. Appl. Math. 11 (1994) 155-170.

[24] S. Matsui, T. Shirota, On separation points of solutions to Prandtl boundary layer problem. Hokkaido Math. J. 13 (1984) 92-108.

[25] A. Mazzucato, M. Taylor, Vanishing viscosity limits for a class of circular pipe flows. Comm. Partial Differential Equations 36 (2011) 328-361.

[26] L. Nirenberg, An abstract form of the nonlinear Cauchy-Kowalewski theorem. J. Differential Geometry 6 (1972) 561-576.

[27] T. Nishida A note on a theorem of Nirenberg. J. Differential Geometry 12 (1977) 629-633.

[28] R. Nguyen van yen, M. Farge, K. Schneider, Energy dissipating structures produced by walls in two-dimensional flows at vanishing viscosity. Phys. Rev. Lett. 106 (2011) 184502.

[29] O. A. Oleinik, On the mathematical theory of boundary layer for an unsteady flow of incompressible fluid. J. Appl. Math. Mech. 30 (1966) 951-974.

[30] P. Orlandi, Vortex dipole rebound from a wall. Phys. Fluids A 2 (1990) 1429.

[31] M. V. Safonov, The abstract Cauchy-Kovalevskaya theorem in a weighted Banach space. Comm. Pure and Appl. Math. 48 (1995) 629-637.

[32] M. Sammartino, R. E. Caflisch, Zero viscosity limit for analytic solutions of the Navier-Stokes equation on a half-space I, Existence for Euler and Prandtl equations. Comm. Math. Phys. 192 (1998) 433-461.

[33] M. Sammartino, R. E. Caflisch, Zero viscosity limit for analytic solutions of the Navier-Stokes equation on a half-space II, Construction of the Navier-Stokes solution. Comm. Math. Phys. 192 (1998) 463-491.

[34] V. A. Solonnikov, Estimates of the solutions of a nonstationary linearized system of Navier-Stokes equations. Amer. Math. Soc. Transl. 75(2) (1968) 1-116.

[35] R. Temam, X. Wang, On the behavior of the solutions of the Navier-Stokes equations at vanishing viscosity. Ann. Scuola Norm. Sup. Pisa Cl. Sci. Volume dedicated to the memory of E. De Giorgi. 25(4) (1998) 807-828.

[36] S. Ukai, A solution formula for the Stokes equation in $\mathbb{R}_{+}^{n}$. Comm. Pure Appl. Math. 11 (1987) 611-621.

[37] X. Wang, Kato type theorem on zero viscosity limit of Navier-Stokes flows. Indiana Univ. Math. J. 50 (2001) 223-241.

[38] W. Wolibner, Un theorème sur l'existence du mouvement plan d'un fluide parfait, homogène, incompressible, pendant un temps infiniment long. Math. Z. 37 (1933) 698-726.

[39] V. I. Yudovich, Non-stationary flows of an ideal incompressible fluid. Ž. Vyčisl. Mat. i Mat. Fiz. 3 (1963) 1032-1066. 